\title{
Interpretable machine learning for psychological research: Opportunities and pitfalls
}

\author{
Mirka Henninger, Rudolf Debelak, Yannick Rothacher, \& Carolin Strobl \\ University of Zurich
}




\section{Author Note:}

Correspondence concerning this article should be addressed to Mirka Henninger, Institute of Psychology, University of Zurich, 8050 Zurich, Switzerland Email: mirka.henninger@uzh.ch. Parts of this work have been presented at the $15^{\text {th }}$ Meeting of the Section of Methodology and Evaluation of the German Psychological Society, Mannheim, Germany and at the $86^{\text {th }}$ Annual

International Meeting of the Psychometric Society, Bologna, Italy. A preprint of this manuscript is available on the PsyArXiv: https://psyarxiv.com/xe83y.

The code behind the simulation studies has been made publicly available at OSF:

https://osf.io/zgc9w. 


\begin{abstract}
In recent years, machine learning methods have become increasingly popular prediction methods in psychology. At the same time, psychological researchers are typically not only interested in making predictions about the dependent variable, but also in learning which predictor variables are relevant, how they influence the dependent variable, and which predictors interact with each other. However, most machine learning methods are not directly interpretable. Interpretation techniques that support researchers in describing how the machine learning technique came to its prediction may be a means to this end. We present a variety of interpretation techniques and illustrate the opportunities they provide for interpreting the results of two widely used black box machine learning methods that serve as our examples: random forests and neural networks. At the same time, we illustrate potential pitfalls and risks of misinterpretation that may occur in certain data settings. We show in which way correlated predictors impact interpretations with regard to the relevance or shape of predictor effects and in which situations interaction effects may or may not be detected. We use simulated didactic examples throughout the article, as well as an empirical data set for illustrating an approach to objectify the interpretation of visualizations. We conclude that, when critically reflected, interpretable machine learning techniques may provide useful tools when describing complex psychological relationships.
\end{abstract}




\section{Introduction}

One of the main goals in psychological research is to describe psychological processes through statistical models. Very often, standard statistical models that are used in psychological research assume linear relationships between predictor variables (in the following "predictors") and dependent variables (in the following "outcomes"). However, many psychological processes may not be well described by a linear model, and are the result of unknown complex interaction effects based on many influential predictors. In this regard, Breiman (2001b) stated that the more complex the prediction we want to make, the less we can use simple and interpretable functions. Detecting and predicting such complex processes requires more flexible approaches like machine learning methods. For these reasons, black box machine learning methods such as random forests and neural networks have become more and more popular in psychology (see e.g., Fokkema \& Strobl, 2020; Gonzalez, 2021; Hong et al., 2020).

Machine learning methods use complex functions to account for the influence of many different predictors and their interactions when predicting the outcome (see e.g., Hastie et al., 2008; Hornik et al., 1989). There are different views on what the term machine learning might comprise. For instance, while most statisticians would probably not classify parametric models with a pre-defined functional form, such as logistic regression, under the term machine learning, many computer scientists do. Within the class of machine learning methods, some are inherently interpretable even though their functional form is not pre-defined. For example decision trees are easily interpretable by visual inspection. Other machine learning methods, such as the ensemble methods bagging and random forests, provide more smooth predictions, but at the cost of giving up the interpretability of single trees. Machine learning methods whose results are not directly interpretable are called black box methods. In this article, we present and discuss interpretation techniques for black box machine learning methods that do not provide any interpretable function. As such, there is no straightforward interpretation of the fitted black box, for instance it is typically impossible to directly evaluate the contribution and relevance of certain predictors on the outcome (Ribeiro et al., 2016; Zhao \& Hastie, 2019).

The interpretation of machine learning methods may be subordinate when the main research goal focuses on prediction. However, in psychological research, we typically aim at gaining knowledge about the underlying processes and at judging which predictors are relevant.

Particularly when high-stakes decisions, such as educational decisions, are made, it is essential that people affected by that decision can understand, trust, and accept the model's predictions (Molnar, 2019; Ribeiro et al., 2016).

To explain predictions of machine learning methods, different interpretation techniques have been proposed in recent years. The interpretation techniques aim at describing the relevance of predictors, the shape of the effect of predictors, the presence of interactions, or at describing how predictions were made for specific persons (e.g., Goldstein et al., 2015; Molnar, 2019; Zhao \& Hastie, 2019). These methods come from various fields, such as statistics or computer sciences, using very different notations in the form of classical formulas or pseudo-code (abbreviated representation of an algorithm). Therefore, besides describing the interpretation techniques theoretically (like e.g., Molnar, 2019), one major contribution of this article is that we present all methods in one joint notation (see Appendix A for a glossary). As another major contribution, we illustrate potential pitfalls where we fear the methods may lead to false conclusions when applied, for example, in psychological research settings. We show that certain data situations can lead to misinterpretation of the importance of predictors, the shape of effects of predictors, or erroneously may mask or suggest interaction effects. With this article, we do not only aim at addressing potential users of these methods, but also motivate researchers to critically reflect on machine 
learning and interpretation techniques when encountering these as readers of psychological research articles.

For large parts of the article, we use simulated data with, for example, induced correlations or induced interaction effects in order to demonstrate how the machine learning and interpretation techniques react to such data settings. Our goal is to present and illustrate these methods lucidly and systematically using data in which we can control the data generating process. Therefore, we have deliberately focused on simple, simulated examples and avoided using empirical data for large parts of the article. We will however use empirical data when we illustrate how direct comparisons between visualizations can be facilitated using a visual inference technique.

\section{Machine Learning Methods and Interpretation Techniques}

In the following, we will illustrate the interpretation techniques for two types of machine learning methods: conditional random forests and feed-forward neural networks. The reasons for this selection are three-fold. First, random forests and neural networks have become popular methods in the social sciences, making it likely that researchers will encounter these methods when reading psychological research articles (see e.g., Bates \& Jacobs, 2020; Gonzalez, 2021; Goretzko \& Bühner, 2020; Weidman et al., 2019, for examples). Second, compared to other machine learning approaches, these machine learning methods are relatively straightforward to implement in the statistical programming environment $R$, making them ready-to-use for psychological researchers.

Third, both machine learning methods have in common that the relationship between predictors and the outcome does not have to be specified or known before the analysis. Neural networks and random forests can learn the complex functional form from the data (see e.g., Hornik et al., 1989; Strobl, Malley, et al., 2009). In contrast, parametric regression models require the researchers to define the functional form by including, for example, polynomial functions.

As we focus on the interpretation techniques in this article, we keep the description of the machine learning methods relatively short. We do, however, highlight some characteristics of random forests and neural networks that are important to understand the effects of the machine learning methods and data settings on the results of the interpretation techniques that we will show below.

\section{Random Forests}

A random forest is a so-called ensemble method that consists of a large number of decision trees as its building blocks (Breiman, 2001a). Each tree is based on the principle of recursive partitioning: by means of a series of splits in the predictors, various subgroups of persons are created (Breiman et al., 1984). The goal in a decision tree is to find subgroups that are homogeneous with respect to the outcome, and the characteristics of each subgroup are described by the tree's splits. As an advancement of decision trees, random forests average over multiple (e.g., 500) trees to make a prediction. Averaging across multiple, diverse trees results in lower error rates and more stable predictions overall (Breiman, 2001a, see also Philipp et al., 2018, for a discussion of tree stability). As is illustrated in Panel A in Figure 1, the individual trees in a random forest can have different structures and contain different predictors in different positions. This makes it virtually impossible to directly summarize for the entire forest how an individual predictor affects the prediction ${ }^{1}$.

A further characteristic of random forests is that each tree is based on a subsample of persons and predictors. As a consequence of sampling from the persons, prediction accuracy can be

\footnotetext{
${ }^{1}$ For visibility, we only show a handful of simplified trees in Figure 1, where a real random forest would contain hundreds of more complex trees.
} 
assessed by means of a so-called out-of-bag error: As each tree uses only parts of the data, the remaining part can be used to assess the tree's prediction accuracy on fresh data (similar to cross-validation). Random forests have two main tuning parameters: the number of trees (in general more trees lead to better performance) and the number of randomly pre-selected predictors to choose from in each split of the tree $(\mathrm{mtry})^{2}$. For an extensive introduction and further details on tuning parameters see Strobl et al., 2009, Probst et al. (2019), and Segal (2004, for a discussion of role of tree depth in large samples and for many predictors).

\section{(A) Random Forest}
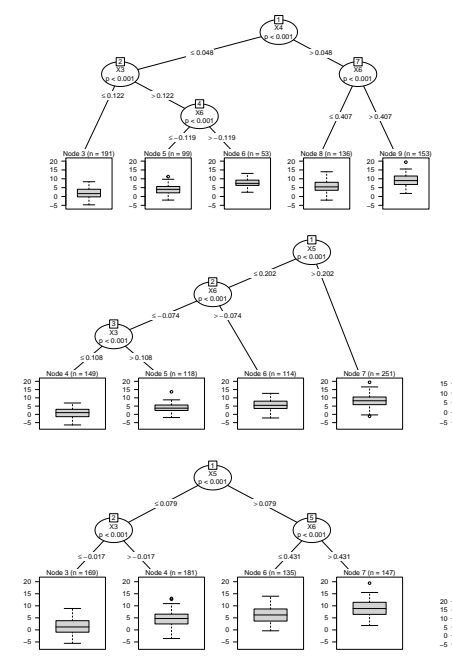
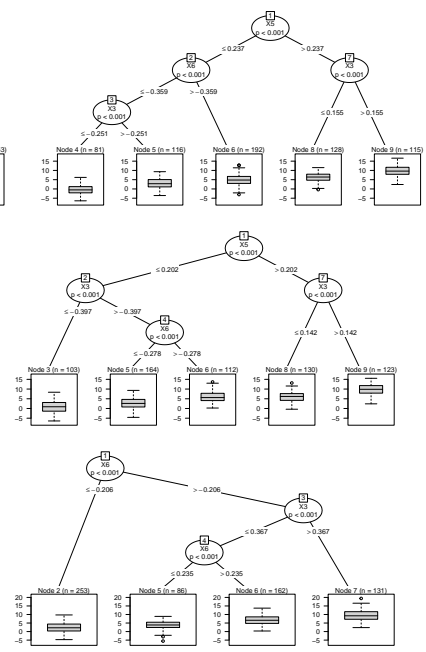

\section{(B) Neural Network}

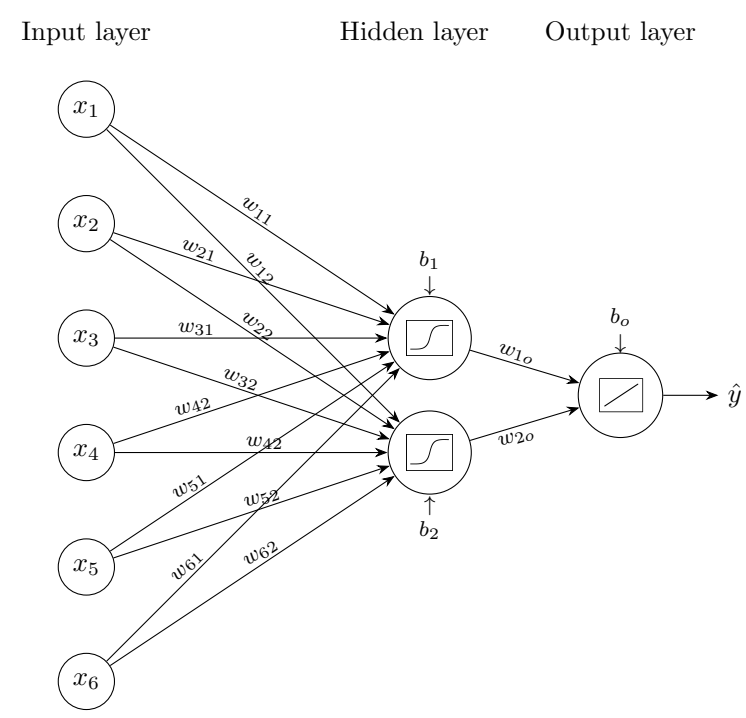

Figure 1: Illustration of $(\mathrm{A})$ the ensemble principle in random forests where multiple decision trees are combined to predict the outcome and (B) the input, hidden and output layer of a neural network with connection weights, a sigmoid activation function in the hidden layer and a linear activation function in the output layer

It has been shown that traditional random forests can be biased in favor of predictors offering more potential cutpoints, which is particularly likely when the data contains predictors of different types, such as categorical and continuous variables (Hothorn et al., 2006; Kim \& Loh, 2001; Strobl et al., 2006). In such a data setting, building random forests based on a conditional inference framework (Hothorn et al., 2006) together with subsampling provides more reliable results than traditional random forest algorithms together with bootstrap sampling (Strobl et al., 2007). We highly recommend this approach for practical applications, where predictors of different types are the rule rather than the exception. To highlight this, we employ it throughout this paper, even though for all our simulation settings, in which only continuous predictors are used, variable selection bias is not an issue.

\section{Neural Networks}

Neural networks are a computational model inspired by the working mechanisms of the biological nervous system. A simple, but widely used architecture for neural networks is a single hidden layer

\footnotetext{
${ }^{2}$ mtry should be tuned, but is often set to $\frac{J}{3}$ by default for regression or $\sqrt{J}$ for classification (with $J$ being the total number of predictors), as evident in the original Random Forest Fortran code (Breiman \& Cutler, 2002), Breiman's Wald lecture series (Breiman, 2002a), and is also suggested by Liaw and Wiener (2002).
} 
feed-forward network, but many other and more complex architectures are possible (Hastie et al., $2008)^{3}$. As illustrated in Panel B in Figure 1, the artificial "neurons" of a neural network are typically organized in three types of layers. The input layer (where predictors are represented by individual neurons), the hidden layer/s (responsible for extracting and processing information with one or multiple neurons) and the output layer (where the predicted outcome is represented by one or multiple neurons).

The neurons in each layer are linked to neurons in neighboring layers through weighted connections. The connection weights $\left(w_{j h}\right.$ and $w_{h o}$, similar to regression weights) and bias terms $\left(b_{1}, b_{2}\right.$, and $b_{o}$, similar to regression intercepts; see Figure 1$)$ determine how predictor values are processed. Training of a neural network is based, for example, on stochastic gradient descent, which involves the iterative adjustment of the connection weights and bias terms until a good prediction of the outcome is reached.

In addition to connection weights and bias terms, there are various tuning parameters which affect the structure and general behavior of the neural network. Examples of tuning parameters include the number of hidden layers and the number of hidden neurons $(H)$, or regularization terms ${ }^{4}$. These tuning parameters directly determine the flexibility of a neural network and must be tuned in order to prevent under- or overfitting. In addition, activation functions transform the incoming information in the neurons of the hidden and output layers, and can introduce non-linearities to the predictive function. The sigmoid function is a popular choice for the activation function in the hidden layer, while the activation function for the output layer depends on the type of the outcome. Alternative choices of activation functions are, for example, the tangent hyperbolic (tanh) function, piecewise linear functions (rectified linear activation function or ReLU), or variants thereof (see e.g., Hastie et al., 2008; Schmidt-Hieber, 2020).

As noted above, neural networks tend to under- or overfit when they are not carefully tuned (e.g., Hastie et al., 2008). This is in contrast to random forests, which have the advantage that they often work well "off the shelf", meaning that they are quite robust against a suboptimal choice of mtry (e.g., Probst et al., 2019, but see also Strobl et al., 2008 and our own results further below for the impact of mtry when predictors are correlated).

The workflow of neural networks, in which the input is passed through multiple subsequent neurons in parallel, renders the interpretation of the predictions difficult. In particular when the number of neurons in the input and hidden layer is high, or multiple hidden layers are used, it is almost impossible to gain a good understanding of the relationship between the individual predictor variables and the outcome (Bates \& Jacobs, 2020; Hastie et al., 2008). Therefore, like random forests, neural networks can be regarded as "black box" machine learning methods.

\section{Interpretation Techniques}

As black box methods, neither random forests nor neural networks allow us to see how the machine learning method has come to a certain prediction. For this purpose, interpretation techniques have been proposed: while we cannot open and interpret the black box itself, we can assess how the black box reacts in different scenarios or with different input parameters, which helps us to infer how decisions have been made inside the black box (Scholbeck, 2018; Štrumbelj \&

\footnotetext{
${ }^{3}$ In the past years, neural network architectures have become more complex, and the simple neural network presented here might not represent the (deep convolutional) networks that data scientists would use in practice, for example for time series prediction or image classification. For the purpose of illustrating some characteristics of neural networks and and compare them to random forests, however, the simple neural network is sufficient.

${ }^{4} \mathrm{~A}$ variety of rules of thumb exist based on the number of neurons in the input layer (i.e. the number of predictors $J$ ) and the number of neurons in the output layer (i.e. $O$ ), such as that the number of neurons in the hidden layer $(H)$ should be $\frac{J+O}{2}, \frac{2}{3} \cdot(J+O), \sqrt{J \cdot O}$, smaller than $J$ or lie between $O$ and $J$ (Heaton, 2008).
} 
Kononenko, 2014).

Many interpretation techniques are independent of the type of the machine learning method. These so-called model-agnostic techniques can be used to illuminate the black box of random forests, neural networks, and many other machine learning methods. Other interpretation techniques are specific to certain machine learning methods, because they use model-specific information. For example, some variable importance measures use the information from the individual trees of a random forest, or the weights between neurons in a neural network. Therefore, these interpretation techniques are specific for these particular machine learning methods.

Another distinction is that between global and local interpretation techniques. In this article, we focus on global techniques. In contrast to local (or person-level) techniques, which describe predictions at certain locations or predictions for specific persons, global techniques describe overall (or sample-level) predictor effects and can, for example, be used to answer research questions with regard to the overall importance of predictors or whether predictors are involved in interaction effects, which are typical aims in psychological research.

\section{Structure of this Article}

We will structure the presentation of the interpretation techniques and our illustration of potential pitfalls in two parts: In part I, we present interpretation techniques that describe the importance and shape of predictor effects, and highlight consequences of correlated predictors; in part II, we present methods for detecting interaction effects and demonstrate in which situations they could erroneously find interaction effects that do not exist, and miss interaction effects that do exist.

\section{General Setup for Data Generation and Model Fitting.}

In both parts, we simulate data in order to illustrate the interpretation techniques in specific data scenarios. We set the sample size to $N=1000$ persons and draw the values of six predictors from a uniform distribution, $\boldsymbol{X} \sim U(-1,1)$. The main data generating process is a linear regression model with the intercept $\beta_{0}=5$, different regression weights for the six predictors, $\boldsymbol{\beta}=(0,2,3,0,2,3)$, and normally distributed errors with $\sigma_{\varepsilon}=2$. This corresponds to standardized regression weights of approximately $\boldsymbol{\beta}^{*}=(0,0.32,0.48,0,0.32,0.48)$. This setup is much simpler and abstract than what we expect in most real psychological studies. However, we will see further below that even this simple setup can reveal potential pitfalls of the interpretation techniques, such as that some of the techniques reflect marginal rather than partial effects of the predictors and vice versa.

In part I, we generate the data with only main effects, and study the effect of strong correlations (setting $\rho$ to either $\rho=0$ or $\rho=.8$ ) between the predictors $x_{4}, x_{5}$, and $x_{6}$. Note that we have chosen this rather strong correlation for reasons that will become clear below.

$$
y_{i}=5+0 \cdot x_{i 1}+2 \cdot x_{i 2}+3 \cdot x_{i 3}+0 \cdot x_{i 4}+2 \cdot x_{i 5}+3 \cdot x_{i 6}+\varepsilon_{i}
$$

$$
\boldsymbol{\Sigma}=\left(\begin{array}{llllll}
1 & 0 & 0 & 0 & 0 & 0 \\
0 & 1 & 0 & 0 & 0 & 0 \\
0 & 0 & 1 & 0 & 0 & 0 \\
0 & 0 & 0 & 1 & \rho & \rho \\
0 & 0 & 0 & \rho & 1 & \rho \\
0 & 0 & 0 & \rho & \rho & 1
\end{array}\right)
$$


In part II, we add an additional predictor $x_{z}$ that interacts with each of the predictors $x_{4}, x_{5}$, and $x_{6}$, but use uncorrelated predictor variables to reduce the complexity of the results.

$$
\begin{gathered}
y_{i}=5+0 \cdot x_{i 1}+2 \cdot x_{i 2}+3 \cdot x_{i 3}+0 \cdot x_{i 4}+2 \cdot x_{i 5}+3 \cdot x_{i 6}+ \\
3 \cdot x_{i 4} \cdot x_{z}+3 \cdot x_{i 5} \cdot x_{z}+3 \cdot x_{i 6} \cdot x_{z}+\varepsilon_{i}, \\
\boldsymbol{\Sigma}=\left(\begin{array}{ccccccc}
1 & 0 & 0 & 0 & 0 & 0 & 0 \\
0 & 1 & 0 & 0 & 0 & 0 & 0 \\
0 & 0 & 1 & 0 & 0 & 0 & 0 \\
0 & 0 & 0 & 1 & 0 & 0 & 0 \\
0 & 0 & 0 & 0 & 1 & 0 & 0 \\
0 & 0 & 0 & 0 & 0 & 1 & 0 \\
0 & 0 & 0 & 0 & 0 & 0 & 1
\end{array}\right)
\end{gathered}
$$

This corresponds to standardized regression weights of approximately $\boldsymbol{\beta}^{*}=(0,0.20,0.29,0,0.20,0.29)$ for the main effects and $\beta^{*}=(0.29)$ for the interaction effects.

\section{Machine Learning Method Setup and Parameter Tuning}

We conducted a pilot study with $R=100$ replications to identify the best architecture and tuning parameters for random forests and neural networks for the two data settings, which were then used in our main simulation studies. We used the partykit package (Hothorn \& Zeileis, 2015) to tune and fit the random forests ${ }^{5}$, and the caret and nnet packages to tune and fit the neural networks (Kuhn, 2020; Venables \& Ripley, 2002). For random forests, we assessed model performance for values of mtry between 1 and the number of predictors ( 6 in part I and 7 in part II). For neural networks, we examined model performance between 1 and the number of predictors times 2 for the number of neurons in the hidden layer (12 in part II and 14 in part II) and values between 0 and 0.2 in steps of 0.05 for regularization. We used a sigmoid activation function in the hidden layer and the linear function in the output layer which is the classic setup for continuous outcomes.

In part I with only main effects in the data generating process and two levels of correlations $\rho \in\{0,0.8\}$, we found a random forest with four predictor variables randomly pre-selected in each split (mtry $=4$; average proportion of variance explained $\overline{R^{2}} \geq 0.64$ ) and a neural network with one neuron in the hidden layer $\left(H=1 ; \overline{R^{2}} \geq 0.68\right)$ to fit a test dataset best in a majority of replications. In part II including additional interaction effects, we found a random forest with seven predictor variables randomly pre-selected in each split (mtry $\left.=7 ; \overline{R^{2}}=0.69\right)$ and a neural network with 9 neurons in the hidden layer $(H=9)$ and a regularization of $0.05\left(\overline{R^{2}}=0.86\right)$ to provide the best fit to a test dataset ${ }^{6}$. In part I and II, we set the number of trees to ntree $=500$ trees in each random forest. For the neural network, there was one neuron per predictor $j$ in the input layer and one neuron for the outcome in the output layer.

For visualizing the interpretation techniques in this article, we used wrapper functions around functions from the R package iml (Molnar et al., 2018). These wrapper functions can be found and

\footnotetext{
${ }^{5}$ The cforest function in the partykit or party package can be used to fit random forests from the conditional inference framework, while the randomForest function from the randomForest package fits the classical random forest that is biased in favor of predictors offering more potential cutpoints.

${ }^{6}$ In the settings investigated here, neural networks provide a slightly better fit than random forests. This is due to the characteristics of the data generating processes. The random forest must approximate the linear data generating process by a large number of splits. In contrast, the neural network can more directly map the linear data generating process used here. Other data generating processes, such as a step function, would yield an advantage to random forests over neural networks.
} 
installed from Github ${ }^{7}$ and may facilitate applying the interpretation techniques in one's own research. The code behind the simulation studies has been made publicly available at $\mathrm{OSF}^{8}$.

\section{Part I: Importance and Shape of Predictor Effects}

In part I we will illustrate interpretation techniques that describe the shape and the relevance of effects of predictors: Partial Dependence (PD), Individual Conditional Expectation (ICE), and Accumulated Local Effect (ALE) plots as well as variable importance measures. While PD, ICE, and ALE plots illustrate the direction, shape, and strength of the effect of a predictor, variable importance measures provide us with a numeric value of the overall relevance of a predictor.

Some of the interpretation techniques are explicitly designed for uncorrelated predictors, such as PD and ICE plots. Other interpretation techniques have been shown to be affected by correlations between predictors, such as the permutation variable importance in random forests (Debeer \& Strobl, 2020; Nicodemus \& Malley, 2009; Nicodemus et al., 2010; Strobl et al., 2008). However, in practice it is unlikely that all predictors are independent from each other. Therefore, besides presenting the methods, we will highlight the effects of correlations between predictors on the interpretation techniques.

\section{Partial Dependence, Individual Conditional Expectations, and Accumulated Local Effects Plots}

PD (Friedman, 2001), ICE (Goldstein et al., 2015), and ALE (Apley \& Zhu, 2020) plots aim at showing the shape of the effect of a predictor (or a pair of predictors, as will be shown in part II) on the outcome. Hence, these plots can be used to assess whether the predictor has, for example, a roughly linear, u-shaped, or more complex relationship with the outcome (Friedman \& Popescu, 2008; Goldstein et al., 2015; Molnar, 2019, for examples see Zhao \& Hastie, 2019). Figure 2 gives a first impression of PD, ICE, and ALE plots for the linear main effect of $x_{3}$ using data generated presented in Equation 1. All three types of plots allow the researcher to see how the prediction of the outcome (y-axis) changes as a function of the value of the predictor (x-axis).
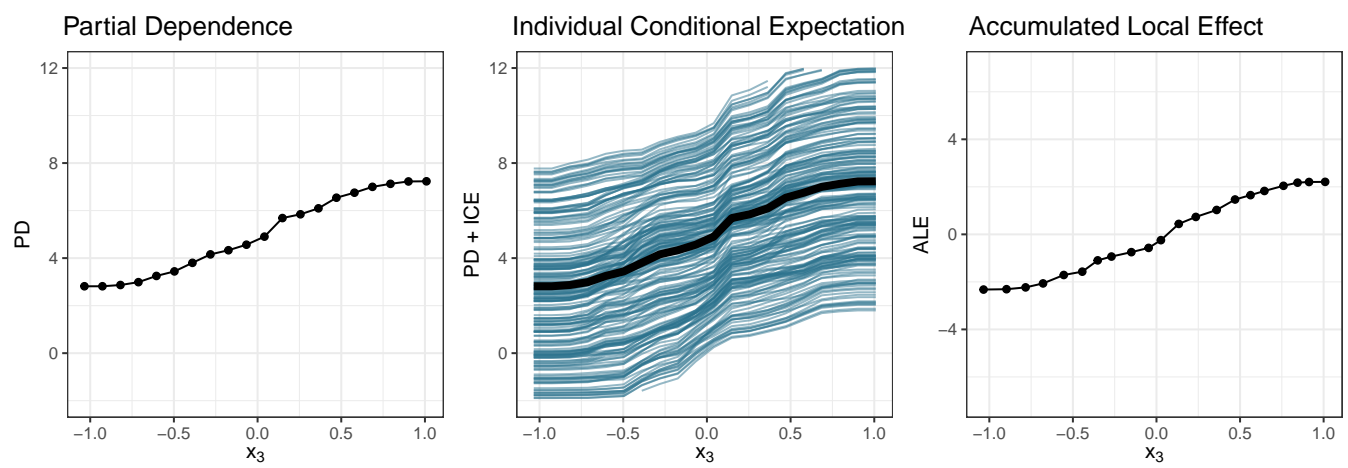

Figure 2: Illustration of Partial Dependence (PD), Individual Conditional Expectation (ICE; with PD in black), and Accumulated Local Effects (ALE) plots of the predictor $x_{3}$ for data generated according to Equation 1 and fitted with a random forest.

We will present PD, ICE, and ALE techniques in detail in this section. In addition to the equations that we provide in the main text, Appendix B illustrates the equations in a graphical

\footnotetext{
${ }^{7}$ https://github.com/mirka-henninger/InterpretableML

${ }^{8}$ https://osf.io/zgc $9 \mathrm{w} /$
} 
way, which can help the interested reader gain en even more thorough understanding of how the interpretation techniques are computed step by step.

\section{Partial Dependence plots}

To draw the PD curve that describes the shape of the effect of predictor $x_{j}$ on the outcome, PD predictions are computed for different positions $\boldsymbol{a}$. Often, the positions $\boldsymbol{a}$ are the observed values of the predictor $x_{j}$ for all persons $i$. But $\boldsymbol{a}$ could also take other values, for example any value in a grid over $x_{j}$, even if not all positions $\boldsymbol{a}$ have actually been observed in the data. To compute PD predictions for a predictor $x_{j}$ for drawing a curve as displayed in Figure 2 (left panel; see also Appendix B.1):

1. Use the trained machine learning method to make a prediction $\hat{y}_{i}$ for each person $i$ for the position $a$ on predictor $x_{j}$, using the observed values of person $i$ for all other predictors.

$$
\hat{y}_{i}=\hat{f}^{M L}\left(x_{i 1}, \ldots, x_{i(j-1)}, a, x_{i(j+1)}, \ldots, x_{i J}\right)
$$

2. Average the predictions over all persons $i$ to obtain the PD prediction of the point $a$. This corresponds to a single point in Figure 2 (left panel).

$$
P D_{j}(a)=\frac{1}{N} \sum_{i=1}^{N} \hat{y}_{i}
$$

3. To obtain $P D_{j}$ predictions over the entire range of predictor $x_{j}$, repeat this procedure for all other positions $\boldsymbol{a}$ and draw the curve. This corresponds to the entire curve in Figure 2 (left panel).

Hence, PD predictions are computed for every person $i$ and every position $a$ on predictor $x_{j}$, using the observed values of person $i$ on all other predictors. This means that PD predictions marginalize over the values of all other predictors in order to show the effect of the predictor $x_{j}$ on the outcome. This also means that when computing the PD predictions, one pretends that every person $i$ had every position $a$ on predictor $x_{j}$ (see also Casalicchio et al., 2019, for an illustration). For instance, person $i$ is passed through all positions on the predictor "age", even though only one actual value on the predictor "age" has been observed for each person. This can be critical, because depending on the distributions of predictors (e.g., extremely skewed) or in case predictors are correlated, PD predictions may be based on combinations of predictor values that are very unlikely or impossible to observe in reality (such as a toddler who graduated from university, or a pensioneer attending preschool). In consequence, the predictions are at least partly based on extrapolating to unobserved values. This principle of extrapolation may remind the reader of the concept of counterfactuals in causality research, where the researcher predicts what would have happened if the person had been in a different condition, and had, for example, received a different treatment (Goldstein et al., 2015).

Figure 3 further illustrates the PD and extrapolation principle with two uncorrelated (left panel) and two correlated (right panel) predictors $x_{1}$ and $x_{2}$ (x-axis and y-axis) coming from a uniform distribution $U(-1,1)$. The color gradient reflects the outcome with higher values being reflected by a lighter color. When a PD curve should be computed for $x_{1}$, for person $i$ (located in the red circle) predictions are made at different positions $a$ (illustrated by the triangles). As $x_{1}$ and $x_{2}$ are independent from each other in the left panel, there are indeed observed combinations of the two predictors close to the different positions $\boldsymbol{a}$. In contrast, in the right panel, predictions 


$$
r\left(x_{1}, x_{2}\right)=0
$$

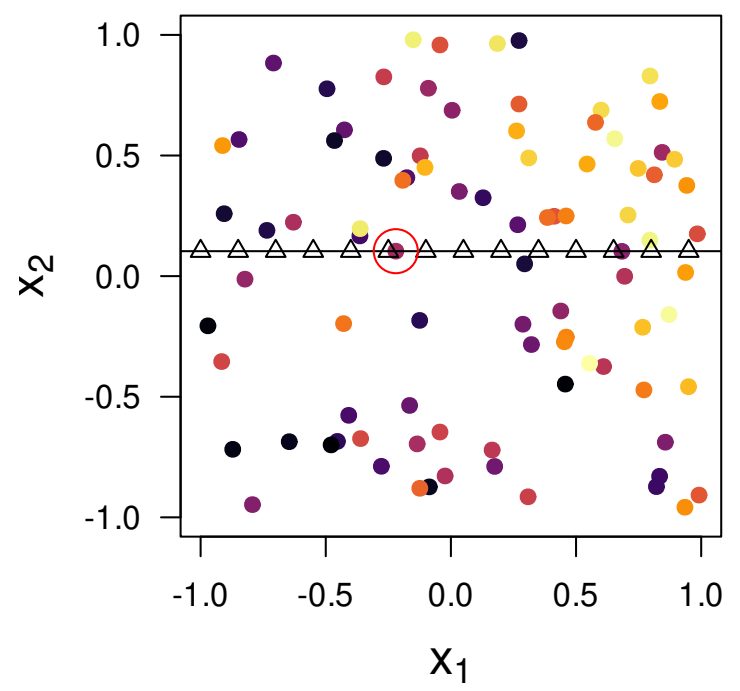

$r\left(x_{1}, x_{2}\right)=.80$

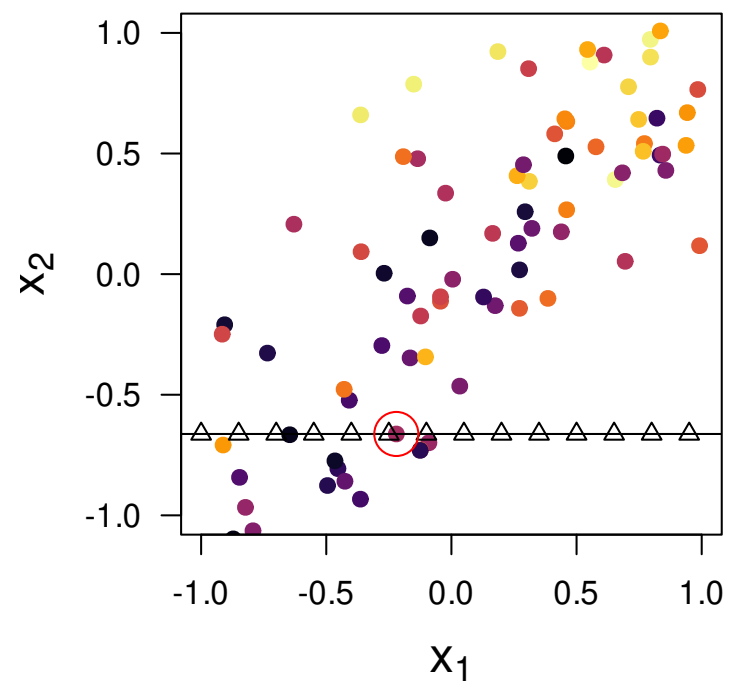

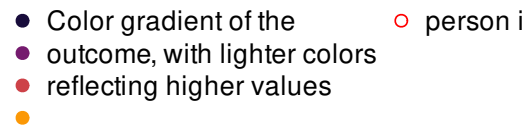

Figure 3: Illustration of the principle underlying Partial Dependence (PD) predictions for two uncorrelated predictors (left panel) and two correlated predictors (right panel).

are made for combinations of values of $x_{1}$ and $x_{2}$ that, due to the strong correlation, would never be observed, such as positive values of $x_{1}$ together with negative values for $x_{2}$ (e.g., Hooker, 2004).

Since PD plots extrapolate to potentially unobserved areas in the predictor space, they have been critized for being unreliable when predictors are correlated (Apley \& Zhu, 2020; Hooker et al., 2021; Molnar, 2019; Molnar et al., 2020; Scholbeck, 2018). Therefore, we will look into the effects of correlated predictors on PD curves further below.

\section{Individual Conditional Expectations Plots}

ICE plots show the predictions of the machine learning method for a predictor $x_{j}$ separately for each person (Figure 2, middle panel). ICE curves can be computed the same way as a PD curve, only without averaging the predictions over persons (leaving out step 2):

1. Use the trained machine learning method to make a prediction $\hat{y}_{i}$ for each person $i$ for the position $a$ on predictor $x_{j}$, using the observed values of person $i$ for all other predictors. This corresponds to single points in the ICE curves in Figure 2 (middle panel).

$$
I C E_{i j}(a)=\hat{f}^{M L}\left(x_{i 1}, \ldots, x_{i(j-1)}, a, x_{i(j+1)}, \ldots, x_{i J}\right)
$$

2. -

3. To obtain $I C E_{i j}$ curves of predictor $x_{j}$, repeat this procedure for all other positions $\boldsymbol{a}$ and draw a curve for every person $i$. This corresponds to the entire set of blue curves in Figure 2. 
The ICE curves for each person can also show how the relationship between the predictor and the outcome depends on other predictors in the model. Without interactions, the ICE curves will all be roughly parallel, as the predictions for $x_{j}$ are independent from the values of other predictors (as in the middle panel of Figure 2). However, when there are interaction effects with other predictors in the data, the curves of different persons will have different slopes and/or shapes (as we will see in part II). For instance, the relationship between $x_{j}$ and $y$ may be positive for some persons, but negative for others, such as when the effect of age on therapy success depends on the type of treatment (Goldstein et al., 2015).

Just like in PD plots, of course, only one point on each curve corresponds to the value of $x_{j}$ that is actually observed for person $i$, while the predictions for all other positions $a$ are based on extrapolations.

\section{Accumulated Local Effects Plots}

To avoid extrapolation which is inherent in PD and ICE plots, Accumulated Local Effects (ALE) plots (right panel in Figure 2) have been proposed by Apley and Zhu (2020). They describe how the prediction of the outcome changes within a small interval of the predictor $x_{j}$. ALE effects for the interval $k$ of the $j^{t h}$ predictor are computed in the following way:

1. The range of predictor $x_{j}$ is split up in $K_{j}$ intervals $A_{j}^{(1)}, \ldots, A_{j}^{\left(K_{j}\right)}$, where each interval $k$ has an upper $\left(a_{j}^{(k)}\right)$ and a lower $\left(a_{j}^{(k-1)}\right)$ interval boundary, so that $A_{j}^{(k)}=\left[a_{j}^{(k-1)}, a_{j}^{(k)}\right]$

2. For $x_{i j}$ that are located within an interval $A_{j}^{(k)}\left(x_{i j} \in A_{j}^{(k)}\right)$, the prediction at the lower interval boundary is subtracted from the prediction at the upper interval boundary.

$$
\begin{aligned}
\Delta_{i}\left(A_{j}^{(k)}\right) & =\hat{f}^{M L}\left(x_{i 1}, \ldots, x_{i(j-1)}, a_{j}^{(k)}, x_{i(j+1)}, \ldots, x_{i J}\right) \\
& -\hat{f}^{M L}\left(x_{i 1}, \ldots, x_{i(j-1)}, a_{j}^{(k-1)}, x_{i(j+1)}, \ldots, x_{i J}\right)
\end{aligned}
$$

3. The prediction differences $\Delta_{i}\left(A_{j}^{(k)}\right)$ are averaged across all $n^{(k)}$ persons located in each interval.

$$
\bar{\Delta}\left(A_{j}^{(k)}\right)=\frac{1}{n^{(k)}} \sum_{x_{i j} \in A_{j}^{(k)}} \Delta_{i}\left(A_{j}^{(k)}\right)
$$

4. In order to describe the shape of the effect of $x_{j}$ on the outcome, the averaged prediction differences are accumulated up to the $k^{\text {th }}$ interval (see Apley \& Zhu, 2020). Hence, the predictor must have some kind of natural order so that these prediction differences can be accumulated sensibly (Molnar, 2019).

$$
A L E^{*}\left(A_{j}^{(k)}\right)=\sum_{k^{*}=1}^{k} \bar{\Delta}\left(A_{j}^{\left(k^{*}\right)}\right)
$$

5. Finally, ALE effects are shifted vertically by subtracting a constant $C_{j}$ so that, averaged across persons, ALE effects are zero. Formally, the constant is a weighted average of each grid interval's ALE effect:

$$
A L E\left(A_{j}^{(k)}\right)=A L E^{*}\left(A_{j}^{(k)}\right)-C_{j}
$$

with

$$
C_{j}=\frac{1}{N} \sum_{k=1}^{K} n^{(k)} A L E^{*}\left(A_{j}^{(k)}\right)
$$

Mathematically speaking, as $\bar{\Delta}\left(A_{j}^{(k)}\right)$ is the prediction change in the interval, one can think of 
the approach as approximating the partial derivative of the prediction function with respect to $x_{j}$ when the number of intervals approaches infinity $\left(K^{(j)} \rightarrow \infty\right)$. Through accumulating (or approximating an integration of) the local effects, one in turn obtains an approximation of the influence of $x_{j}$ on the outcome, but (similar to integral calculus) the information about the absolute level of the outcome is lost (Apley \& Zhu, 2020; Scholbeck, 2018). In Figure 2, the PD curve (left) and the ALE curve (right) look very similar to each other. The main visible difference is that the PD curve directly displays the predicted value of the outcome (such that $P D_{x_{1}}(0) \approx 5$ ), while the ALE curve reflects the change in prediction and therefore provide no information on the absolute level of the outcome $\left(A L E_{x_{1}}(0) \approx 0\right)$.

Figure 4 illustrates the principle of computing ALE effects for $x_{1}$ for two uncorrelated and two correlated predictors. For each observation (in red circles) within the interval boundaries a prediction for the outcome is made on the upper and lower boundary (horizontal arrows). In the right panel, we see the difference in prediction for two correlated predictors. We can see that ALE plots tend not to extrapolate to unobserved values as they only extrapolate within the interval boundaries. The authors state that, therefore, ALE curves should be more reliable than PD or ICE plots when predictors are correlated (Apley \& Zhu, 2020). We will critically review this claim in the upcoming section.

$$
r\left(x_{1}, x_{2}\right)=0
$$

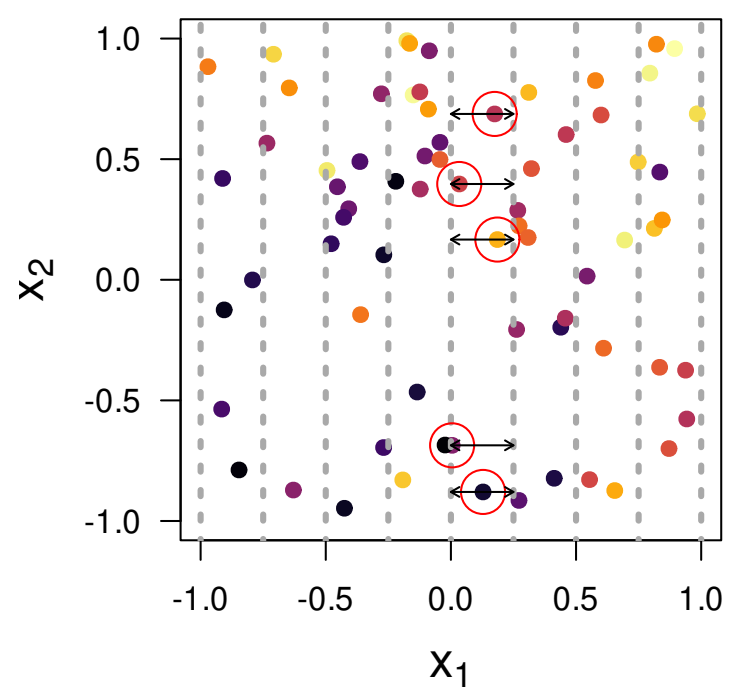

$$
r\left(x_{1}, x_{2}\right)=.80
$$

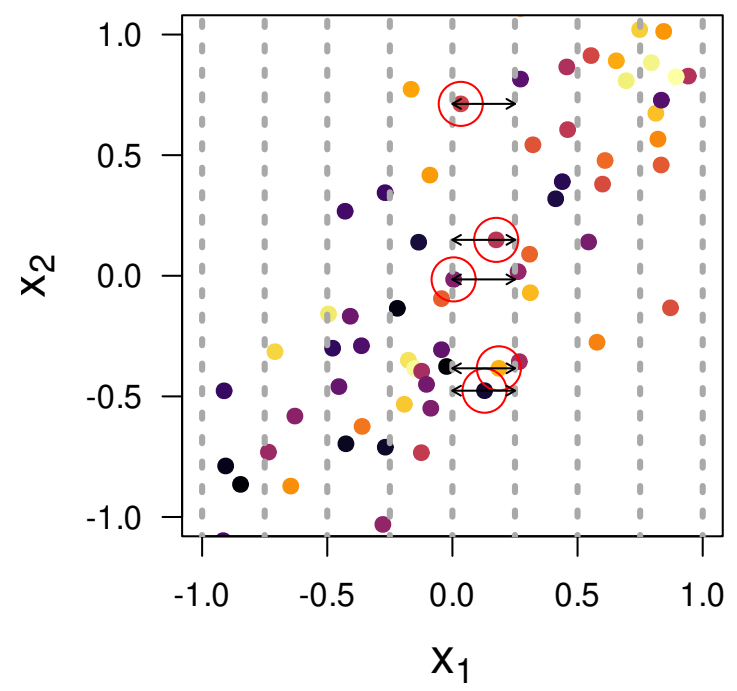

- Color gradient of the $\quad$ persons within a grid cell | interval boundary

- outcome, with lighter colors

- reflecting higher values

$\bullet$

Figure 4: Illustration of the Accumulated Local Effect (ALE) principle for simulated data with two uncorrelated predictors (left panel) and two correlated predictors (right panel).

\section{Correlated Predictors in PD, ICE, and ALE plots}

We examined the effect of strong correlation between predictors using the data generating process according to Equation 1 for $N=1000$ persons, and three uncorrelated $\left(x_{1}, x_{2}, x_{3} ; \rho=.0\right)$ and 
three block-correlated $\left(x_{4}, x_{5}, x_{6} ; \rho=.8\right)$ predictors $^{9}$. For the random forest and the neural network, we computed PD, ICE, and ALE predictions using the R package iml.

Panel A in Figure 5 shows PD (black) and ICE (blue) curves for the random forest. In the first three columns, we see increasing linear effects of $x_{1}, x_{2}$, and $x_{3}$. We also see that in the random forest PD and ICE curves have slightly steeper slopes for the correlated predictors $\left(x_{4}, x_{5}\right.$, and $x_{6}$. However, PD and ICE curves do not substantially differ from ALE curves (Panel B), even for such strongly correlated predictors. Thus, even though ALE plots rely on a different computation scheme, that avoids extrapolating beyond the grid interval's boundaries, the main supporting argument for ALE by its authors (see Apley \& Zhu, 2020) that ALE curves are more reliable than PD curves in case of correlated predictors is not supported here. Apley and Zhu (2020) show that extrapolation in PD plots may lead to different interpretations. However, the effect does only show for very extreme correlations where the variables are almost linearly dependent. Even though the correlation we used here $(\rho=.8)$ is quite high for psychological data settings, we do not find observable differences between PD and ALE curves.

Panels C and D show PD and ICE as well as ALE curves for the neural network. The interpretation of the shape of the effect seems to be even more unaffected by the induced correlations between predictors (left three vs. right three panels). Furthermore, the PD, ICE, and ALE curves are much more even and straight than the curves for the random forest. This is because the simple architecture of the neural network allows to directly reflect the data generating process that was based on a linear model.

\section{What do PD, ICE, and ALE plots show?}

Using the random forest (Panel A in Figure 5), PD, ICE, and also ALE plots suggested stronger effects for the correlated predictors. In particular, they also showed a small, but noticeable effect of the predictor $\left(x_{4}\right)$ when it was correlated to other predictors that had an effect on the outcome $\left(x_{5}\right.$ and $x_{6}$ ), despite the fact that its coefficient in the data generating process was zero just like that of $x_{1}$. The reason for this phenomenon is that PD and ICE plots represent marginal, rather than partial effects. This may come as a surprise, particularly since the terms "Partial Dependence" and "Individual Conditional Expectation" may suggest the opposite. Standard regression model coefficients, which psychological researchers may be more familiar with, do actually reflect partial effects. The estimated regression coefficients of a linear regression model would on average be zero for predictors that have a true null effect in the data generating process, whether they are correlated with other predictors or not. This is because partial effects reflect the effect of a predictor while all other predictors are held constant (note, however, that correlations do inflate the standard errors of regression coefficients, see Azen \& Budescu, 2003; Eid et al., 2015). For PD and ICE plots, this is not the case: The other predictors are not held constant, but averaged over or left to vary between persons, so that PD and ICE plots do not represent partial effects.

The machine learning method itself, that is here whether we look at a plot for a random forest or a neural network, also has an influence on the possible shapes of the effects. For both machine learning methods, the curves are monotonously increasing. At the same time, PD and ALE curves for the random forests are more "wiggly", because random forests need to predict the outcome by aggregating over numerous splits in the decision trees. The neural network, however can reflect the linearity of the data generating process more directly. Hence, the interpretation of the shape of the predictor's effect on the outcome does not only depend on the data generating process, but also on the choice of the machine learning method.

\footnotetext{
${ }^{9}$ as a reminder: $y_{i}=5+0 \cdot x_{i 1}+2 \cdot x_{i 2}+3 \cdot x_{i 3}+0 \cdot x_{i 4}+2 \cdot x_{i 5}+3 \cdot x_{i 6}+\varepsilon_{i}$
} 
Random Forest:

(A) PD + ICE plots
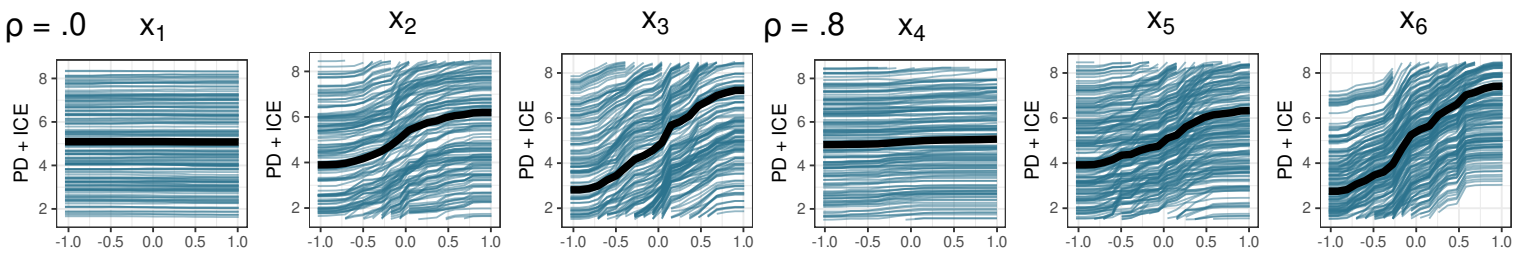

(B) ALE plots

$\rho=.0$

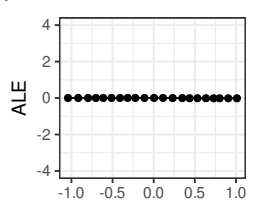

$\beta_{1}=0$

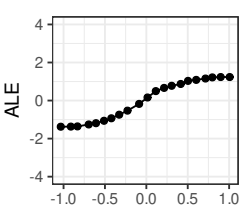

$\beta_{2}=2$

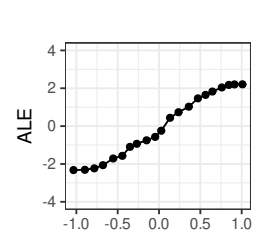

$\beta_{3}=3$ $\rho=.8$

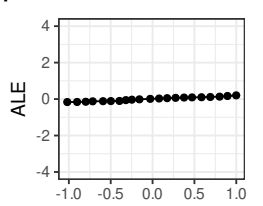

$\beta_{4}=0$

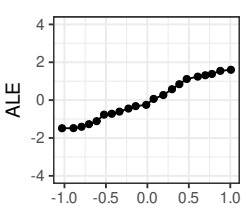

$\beta_{5}=2$

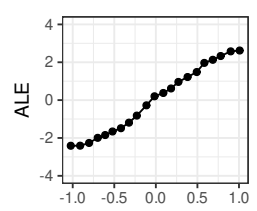

$\beta_{6}=3$

\section{Neural Network:}

(C) PD + ICE plots
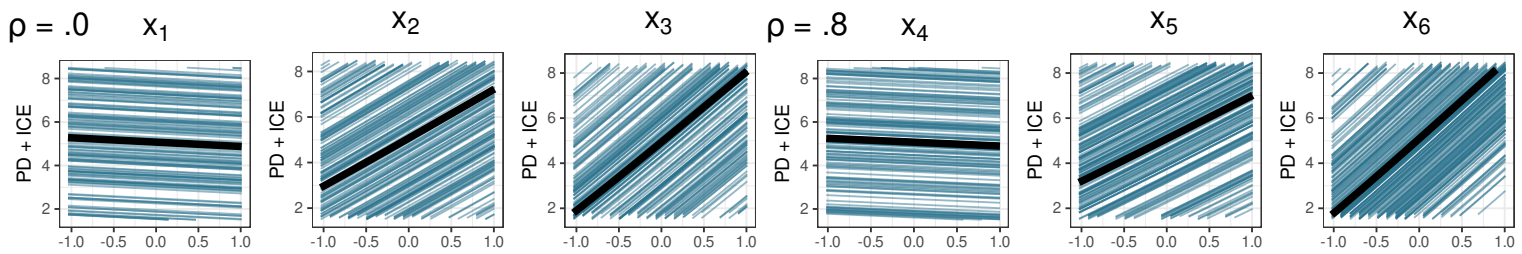

\section{(D) ALE plots}

$\rho=.0$

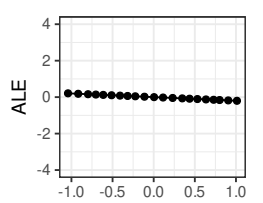

$\beta_{1}=0$

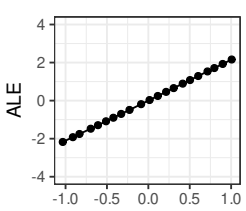

$\beta_{2}=2$

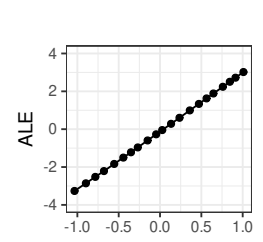

$\beta_{3}=3$ $\rho=.8$

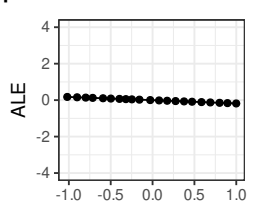

$\beta_{4}=0$

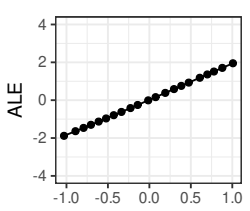

$\beta_{5}=2$

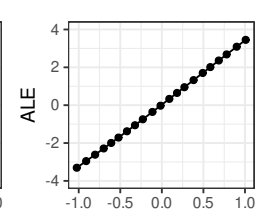

$\beta_{6}=3$

Figure 5: PD, ICE (Panel A and C), and ALE curves (Panel B and D). Data were generated under a linear regression model according to Equation 1 with no correlations between $x_{1}, x_{2}$, and $x_{3}$, while $x_{4}$, $x_{5}$, and $x_{6}$ are block-correlated with $\rho=.8$. PD and ICE curves are displayed with a restricted y-axis, ICE curves are based on a random sample of 200 persons; all visualizations in one row have fixed $\mathrm{y}$-axis scales, and Panel A and $\mathrm{C}$ as well as B and D have the same $\mathrm{y}$-axis limits (ALE plots without fixed scales are shown in Figure C.1 in Appendix C).

\section{How can one tell whether extrapolation is a problem?}

Figure 6 shows bivariate PD plots (which will be introduced in more detail further below) for $x_{1}$ and $x_{3}$ (uncorrelated) as well as $x_{4}$ and $x_{6}$ (strongly correlated) based on a random forest. We have also displayed the univariate distribution by the light grey rugs (grey dashes next to $\mathrm{x}$-axis and y-axis) together with the observed data points. The color gradient reflects the machine 
Independent Predictor Variables

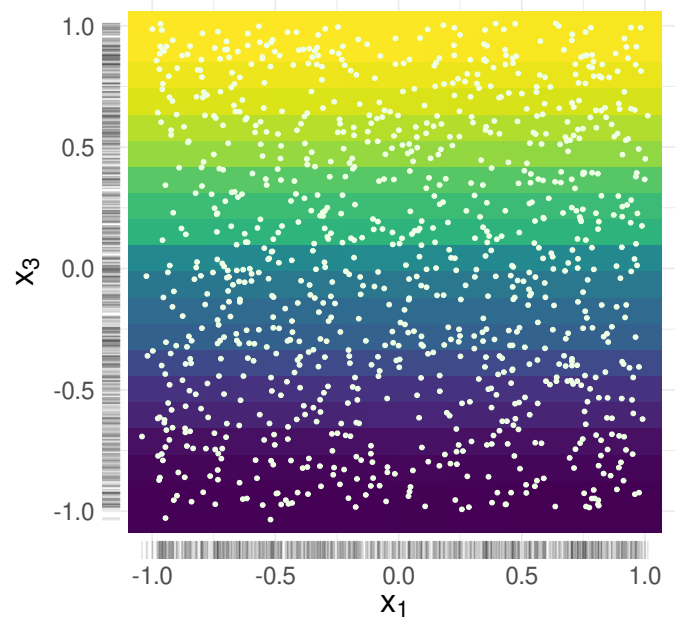

Strongly Correlated Predictor Variables $(\rho=0.8)$

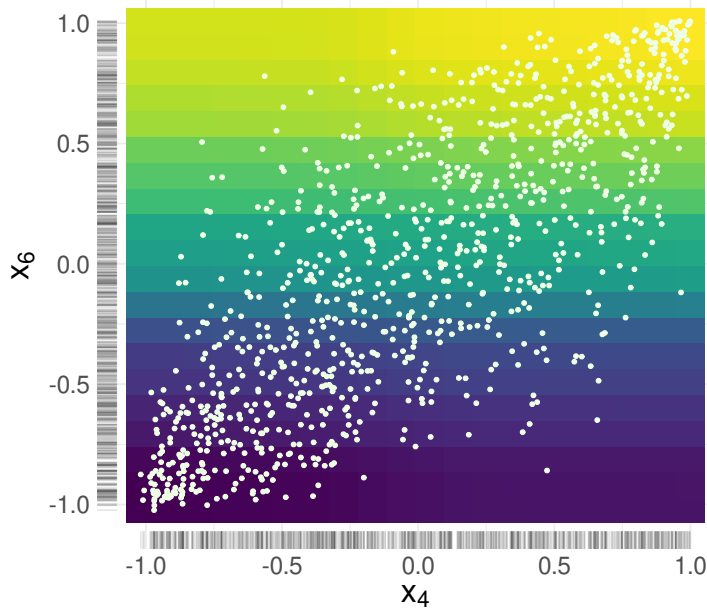

2D PD

7

Figure 6: Two-dimensional PD plot for a random forest illustrating observed data and two-way PD predictions for two independent (left panel) and two correlated (right panel) predictors.

Two-dimensional PD plots will be introduced in more detail further below.

learning method's predictions. These predictions reflect well that $x_{1}$ and $x_{4}$ have no effect on the outcome (no difference from left to right), while $x_{3}$ and $x_{6}$ have an effect on the outcome (color gradient from bottom to top). We can also see that the correlation between $x_{4}$ and $x_{6}$ did not lead to extreme predictions due to extrapolation in the areas where no data was present (top left and bottom right corner of the right panel). However, we see a slight influence of the correlation in the right panel, where the top left corner seems to be a bit greener rather than yellow compared to the upper right corner, suggesting a small main effect of $x_{4}$ (becoming slightly lighter from left to right).

This effect is even more visible when we turn to single decision trees instead of looking at the smooth prediction surface based on a random forest (Figure 6). In Appendix D, we show the bivariate PD predictions for $x_{1}$ and $x_{3}$ as well as $x_{4}$ and $x_{6}$ for a single tree of the random forest. In the upper row, we see that the predictions make more jumps for a single decision tree than for the random forest, and now we also see an effect of $x_{4}$ in the color gradient from left to right. The effect is even more pronounced when mtry is not tuned, but set to its typical default value $\frac{J}{3}=2$. Hence, users may want to interpret PD plots with caution when they find such jumps or strong variation in the predictions for areas where no data is present.

\section{Variable Importance}

Besides the graphical techniques (PD, ICE, and ALE plots) introduced above, variable importance measures can be used to describe the overall importance of each predictor to the prediction made by the machine learning method. An advantage of variable importance measures is that in principle they can capture all main and interaction effects a predictor is involved in without specifying them a priori, while interaction effects in classical regression models need to be specified explicitly to be included in the model (Strobl et al., 2008). However, the measures give neither information about the data generating process in the population nor about the shape or direction of the effect (Casalicchio et al., 2019; Debeer \& Strobl, 2020). In line with that, the importance measures cannot be interpreted as a regression weight and extra steps need to be taken for 
variable selection or significance testing (see e.g., Rothacher \& Strobl, 2022).

There are different types of importance measures. Among the most popular are Gini importance and permutation importance (PI) for random forests (and related ensemble methods; Breiman, 2001a). In addition, Strobl et al. (2008) proposed a conditional permutation importance for random forests. For neural networks, specific importance measures exist, for example based on the connection weights between the input, hidden, and output layers (see Olden et al., 2004, for an overview). In addition, model-agnostic permutation importance measures have been proposed, which can be applied independently of the machine learning method (e.g., Fisher et al., 2019). As the Gini importance has been shown to be biased towards predictors offering many cutpoints, which is often the case when predictors of different types such as categorical, ordinal, and continuous predictors are included in the model (Strobl et al., 2007), here we focus on three permutation importance measures, namely the model-agnostic permutation importance measure (e.g., Fisher et al., 2019, see also Molnar, 2019), the original permutation importance for random forests (Breiman, 2001a), and the conditional permutation importance measure for random forests (Debeer \& Strobl, 2020; Strobl et al., 2008) ${ }^{10}$.

We distinguish between the original and the conditional permutation importance, because we would like to highlight some fundamental conceptual differences between marginal and partial importance measures. While marginal effects reflect the impact of a predictor without controlling for other predictors (similar to zero-order correlations), partial effects reflect the impact of a predictor on top of other predictors in the model (similar to partial regression coefficients; see Azen \& Budescu, 2003; Debeer \& Strobl, 2020; Grömping, 2015, for discussions).

For example, a child's shoe size is highly correlated with their reading ability. We typically consider this a spurious correlation, because we assume that increasing age is the actual driving force behind the increasing reading ability in older kids - who also happen to have larger feet. A fully partial or conditional view would accordingly tell us that shoe size has no additional information once we use age to predict the reading ability, and often research questions in psychology will work in this way. However, it is also a fully legitimate question to ask whether shoe size can be useful for predicting reading ability. This reflects the marginal view and indeed shoe size would do a pretty good job predicting reading ability. If this is our aim, there is nothing wrong with assigning a high importance to shoe size, too.

The original permutation importance aims at reflecting marginal effects, while the conditional variable importance aims at reflecting partial effects. We will see further below how the two importance measure differ from each other when predictors are correlated.

In addition to the permutation importance measures, we present the neural network relative weight importance measure, which is specific to neural networks and is currently implemented as the default variable importance measure for neural networks in the $\mathrm{R}$ package caret (Gevrey et al., 2003; Kuhn, 2020) ${ }^{11}$.

\footnotetext{
${ }^{10} \mathrm{As}$ a side note, we would like to mention that the term "conditional" in the context of the conditional variable importance of Strobl et al. (2008) has a different origin than in the name of the conditional random forest algorithm of Hothorn et al. (2006). Conditional random forests are named after the conditional inference framework of Strasser and Weber (1999, cf. Hothorn \& Zeileis, 2015), which they use for variable selection. The conditional variable importance uses a conditional permutation scheme for computing the variable importance. Both the original and the conditional permutation importance can be combined with both original and conditional random forests. Here, we combine them only with conditional random forests because this algorithm avoids variable selection bias, as discussed above.

${ }^{11}$ There are further techniques for interpreting neural network predictions. A comprehensive overview on measure for tabular data, e.g., is given by Gevrey et al. (2003). For insights into more sophisticated interpretation techniques for neural network, such as layer-wise relevance propagation or spectral relevance analysis see Lapuschkin et al. (2019).
} 
Table 1: Overview of the permutation importance measures, their construction principles, and their implementation in $\mathrm{R}$ packages.

\begin{tabular}{lll}
\hline Type of Permutation Importance & Permute $x_{j}$ & R Package \\
\hline $\begin{array}{l}\text { Model-Agnostic } \\
\text { Permutation Importance }\end{array}$ & for the whole method & iml, vip, ... \\
$\begin{array}{l}\text { Random Forest } \\
\text { Permutation Importance }\end{array}$ & $\begin{array}{l}\text { in each tree and average } \\
\text { across trees }\end{array}$ & $\begin{array}{l}\text { randomForest, party, } \\
\text { partykit, permimp }\end{array}$ \\
$\begin{array}{l}\text { Random Forest } \\
\text { Conditional Permutation Importance }\end{array}$ & $\begin{array}{l}\text { within grid cells of } \boldsymbol{x}_{z} \\
\text { in each tree and average } \\
\text { across trees }\end{array}$ & party, partykit, permimp \\
\hline
\end{tabular}

\section{Permutation Importance}

The general idea of the permutation importance (Breiman, 2001a) is based on computing the difference in prediction accuracy with and without using the information from a certain predictor. The idea is that the prediction becomes worse when the information of an influential predictor is removed from the model. So, the prediction error is calculated twice: once with all predictors in their original form, and a second time with the predictor of interest $x_{j}$ being permuted so that person $i$ receives the value of another person $i^{\prime}$ on the predictor $x_{j}\left(x_{i j}^{\text {permuted }}=x_{i^{\prime} j}\right)$. By "shuffling" the values of $x_{j}$, its association with response $y$ is broken. Hence, the permutation importance of predictor $x_{j}$ can be defined as the difference between (or alternatively fraction of) the original model error and model error with permuted $x_{j}$ :

$$
\mathrm{PI}_{j}=\hat{\varepsilon}^{\text {permuted }}-\hat{\varepsilon}^{\text {original }}
$$

If $\hat{\varepsilon}^{\text {permuted }} \approx \hat{\varepsilon}^{\text {original }}$, this indicates that $x_{j}$ had no influence on the prediction of the machine learning method in the first place. If, however, $\hat{\varepsilon}^{\text {permuted }}$ is notably larger than $\hat{\varepsilon}^{\text {original }}$, this indicates that permuting the predictor $x_{j}$ has destroyed its originally relevant effect in the prediction. To quantify the prediction error of the machine learning method, one can, for example, use the mean squared error of the observed and predicted outcome. Then, the permutation importance of predictor $x_{j}$ can be defined as:

$$
\begin{aligned}
\mathrm{PI}_{j} & =\frac{1}{N} \sum_{i=1}^{N}\left(y_{i}-\hat{f}^{M L}\left(x_{i 1}, \ldots, x_{i(j-1)}, x_{i j}^{\text {permuted }}, x_{i(j+1)}, \ldots, x_{i J}\right)\right)^{2} \\
& -\frac{1}{N} \sum_{i=1}^{N}\left(y_{i}-\hat{f}^{M L}\left(x_{i 1}, \ldots, x_{i(j-1)}, x_{i j}, x_{i(j+1)}, \ldots, x_{i J}\right)\right)^{2}
\end{aligned}
$$

\section{Model-agnostic Permutation Importance (for Random Forests, Neural Networks, etc.). The three types of permutation importance mentioned above are based on different computation schemes that are summarized in Table 1. In the model-agnostic measure, the permutation importance is computed for the whole method. As a consequence, the technique can be applied to any type of machine learning method, such as random forests, but also neural networks (Fisher et al., 2019; Molnar, 2019).}

Original Permutation Importance (for Random Forests). The random forest permutation importance is based on the out-of-bag prediction error in each tree $t$ of the ensemble 
with $t \in(1, \ldots$, ntree $)$. As the permutation importance measures in each tree is then averaged across all trees, it is specific for random forests and cannot be applied to, for example, neural networks.

$$
\mathrm{PI}_{j}=\frac{\sum_{\mathrm{t}=1}^{\mathrm{ntree}} \mathrm{PI}_{j}^{(\mathrm{t})}}{\text { ntree }}
$$

Conditional Permutation Importance (for Random Forests). Strobl et al. (2008) proposed a conditional permutation importance measure that, for each tree, permutes $x_{j}$ conditional on other, correlated predictors $\boldsymbol{x}_{z}$, and then averages across trees to obtain a global measure. The decision which predictors are conditioned on is based on how strongly $x_{j}$ and $x_{z}$ are associated $^{12}$. The computational burden of the conditional permutation importance depends on various factors, such as tree depth, and can become quite large.

The original random forest permutation importance has been shown to assign higher importance to correlated predictors (Nicodemus et al., 2010; Strobl et al., 2008) and behaves more similar to marginal effects or zero-order correlations in linear regression (Debeer \& Strobl, 2020). By contrast, the conditional permutation importance tries to partial out the effects of other predictors that are related to the predictor of interest by means of a conditional permutation scheme. Hence, more similar to the idea of partial regression coefficients or semi-partial correlations, other predictors are attempted to be held constant when the conditional permutation importance is calculated (Debeer \& Strobl, 2020; Strobl et al., 2008).

\section{Relative Weight Importance (for Neural Networks)}

The relative weight importance measure is specific for neural networks and, in contrast to using permutations as an indicator of importance, it weighs the relative importance of predictors against each other (Gevrey et al., 2003). To compute the relative weight importance, the absolute value of the weight $w_{j h}$ between the neuron reflecting predictor $j$ and a neuron $h$ in the hidden layer (see Panel B in Figure 1) is divided by the sum of the absolute values of all the weights of all input neurons connected to a neuron $h$ in the hidden layer:

$$
Q_{j h}=\frac{\left|w_{j h}\right|}{\sum_{j=1}^{J}\left|w_{j h}\right|}
$$

To obtain the relative importance for each predictor $j$, the sum of the $Q_{j h}$ for each neuron $h$ in the hidden layer is divided by the sum over all $Q_{j h}$. As the relative importance is a percentage measure, the result is multiplied by 100 .

$$
\mathrm{RI}_{j}=\frac{\sum_{h=1}^{H} Q_{j h}}{\sum_{h=1}^{H} \sum_{j=1}^{J} Q_{j h}} \cdot 100
$$

\section{Interpretation of Variable Importance Measures}

To illustrate the behavior of the different variable importance measures in general, as well as in particular for correlated predictors, we conducted a simulation study with $R=1000$ replications.

\footnotetext{
${ }^{12}$ The decision which predictors are conditioned on is based on a statistical test that measures the association between $x_{j}$ and $x_{z}$. To this aim, $1-p$ is calculated, where $p$ is the $p$-value of the association test. If this value is larger than a user-defined threshold $s(0 \leq s \leq 1), x_{z}$ is conditioned on when the importance of $x_{j}$ is evaluated. Hence, the smaller the threshold $s$ is chosen, the more predictors are selected to be conditioned on. In contrast, when the threshold is set to $s=1$, no predictors are selected to be conditioned on, and the permutation importance is equivalent to the original random forest permutation importance (see Debeer \& Strobl, 2020; Strobl et al., 2008, for more details)
} 
In each replication, we generated data for $N=1000$ persons based on Equation 1 with three uncorrelated $\left(x_{1}, x_{2}, x_{3}\right)$ and three block-correlated $\left(x_{4}, x_{5}, x_{6}\right)$ predictors. To look at the effect of different correlations between predictors, we set the true correlation $\rho$ between $x_{4}, x_{5}$, and $x_{6}$ to either of $\rho=0$ or $\rho=.8$. To each of the generated $R=1000$ datasets, we fitted a random forest $(\mathrm{mtry}=4)$ and a neural network with one neuron in the hidden layer $(H=1)$ using the tuning parameter identified in the pilot study. We then computed the three permutation importance measures using the R packages iml for the model-agnostic permutation importance (Molnar et al., 2018) and partykit (Hothorn \& Zeileis, 2015) for the non-conditional and conditional random forest variable importance measures. The conditional permutation importance requires to set a threshold value which we set to $s=.95$ as recommended by Debeer and Strobl $(2020)^{13}$. For the neural network, we computed the model-agnostic permutation importance using iml and the relative weight importance using the $\mathrm{R}$ package caret.

Figure 7 shows the values of the importance measures for the different correlations between $x_{4}$, $x_{5}$, and $x_{6}$ across $R=1000$ replications.

\section{Can we interpret the absolute importance values?}

When all predictors are independent from each other (upper row), all variable importance measures result in a similar pattern. We can see that they match the pattern of the true effects in the data generating process (see Equation 1) by assigning equal permutation importance to $x_{1}$ and $x_{4}, x_{2}$ and $x_{5}$, and $x_{3}$ and $x_{6}$, respectively. We can also see that, among others (such as tuning parameters or sample size), the absolute importance values depend on the type of the importance measure. For instance, the conditional importance has overall lower importance values than the marginal importance measures, and the relative weight importance has different distances between predictors. Therefore, the absolute importance values are not comparable between the different measures or between different studies, and we recommend to interpret only the ranking of the most important predictors (see also Probst et al., 2019; Strobl, Malley, et al., 2009). However, the ranks of the top predictors within one measure should be stable when the number of trees is large ${ }^{14}$, whereas the ranks of the uninfluential predictors fluctuate randomly. Different approaches for automatically identifying relevant predictors based on their variable importance have been suggested (see Rothacher \& Strobl, 2022, for an overview and comparison).

\section{What is the effect of correlations between predictors?}

When block-correlations between $x_{4}, x_{5}$, and $x_{6}$ are included in the data generating process (bottom row of Figure 7), the permutation importance measures applied to a random forest yield different patterns for uncorrelated (empty shapes) and correlated (solid shapes) predictors and also between the different permutation importance measures.

Looking at the permutation importance for $x_{4}$, one can see that the conditional importance (third column) reflects that $x_{4}$ has no effect on the outcome in the data generating process by a permutation importance value of 0 , but also substantially diminished for $x_{5}$ and $x_{6}$. In contrast, the model-agnostic permutation importance and the original random forest permutation importance tend to assign a higher importance to $x_{4}$, which has no effect of its own in the data generating model, but is correlated to $x_{5}$ and $x_{6}$ that do have an effect. Similarly, the importance

\footnotetext{
${ }^{13}$ As a complement to their article, Debeer and Strobl (2020) present a Shiny App in which users can examine the effect of different threshold values on conditional permutation importance for multiple data generating processes: https: //simulations-and-statistics.shinyapps.io/CPI-revisited/.

${ }^{14}$ Strobl, Hothorn, et al. (2009) suggest to use this as a check: If the variable importance ranking of the top predictors varies when the forest is refitted after using a different seed for R's random number generator, the number of trees should be increased.
} 


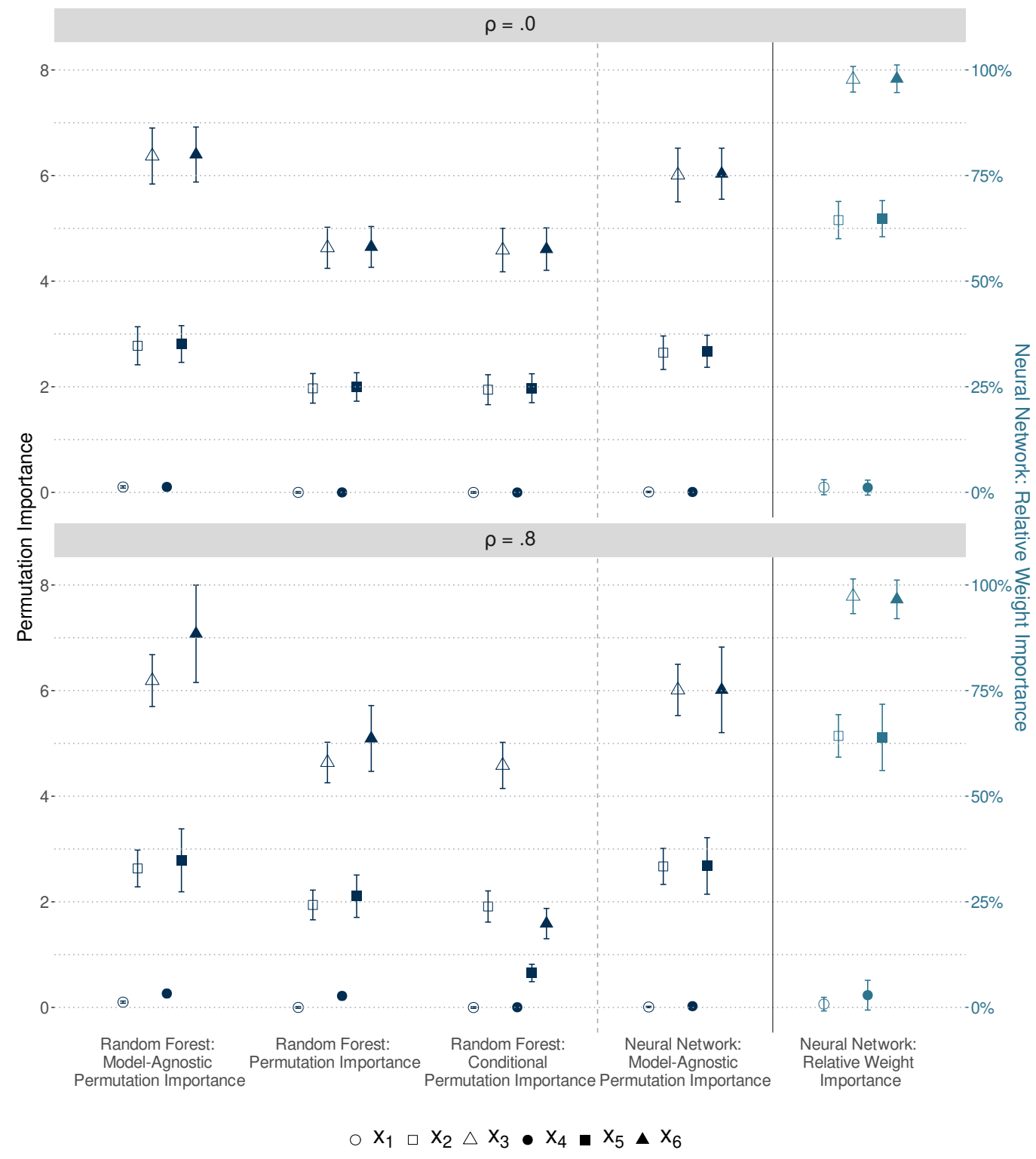

Figure 7: Mean ${ }^{+} /$- 1 standard deviation of variable importance measures for three uncorrelated $\left(x_{1}\right.$, $x_{2}, x_{3}$; empty shapes) and three block-correlated $\left(x_{4}, x_{5}, x_{6}\right.$; solid shapes) predictors across $R=1000$ replications. All predictors are uncorrelated in the upper row. Block-correlations for predictors $x_{4}$ to $x_{6}$ (solid shapes) are set to $\rho=.8$ in the lower row. The data were generated under a linear regression model according to Equation 1. The number of randomly selected predictors in each split was tuned to mtry $=4$.

of correlated predictors $x_{5}$ and particularly $x_{6}$ have higher values than their uncorrelated counterparts $x_{2}$ and $x_{3}$ for these unconditional importance measures (see also Nicodemus \& Malley, 2009; Nicodemus et al., 2010; Strobl et al., 2008, for similar results). Based on previous research (e.g., Strobl et al., 2008), we hypothesized that in particular the effect for $x_{4}$ and $x_{5}$ (the predictors with lower main effects) would be even more pronounced if mtry was not tuned (here the tuned mtry was 4 ), but fixed to the standard value suggested by Breiman $\frac{J}{3}=2$ (Breiman \& Cutler, 2002). Figure 8 shows the results of an additional simulation study we conducted using 
mtry $=2$ for the random forests. Indeed, we see that the original permutation importance measures assigns an even higher importance to the effect of $x_{4}$ and $x_{5}$ than in the setting where mtry has been tuned (Figure 7$)^{15}$, illustrating the interpretation of variable importance also depends on tuning parameters.

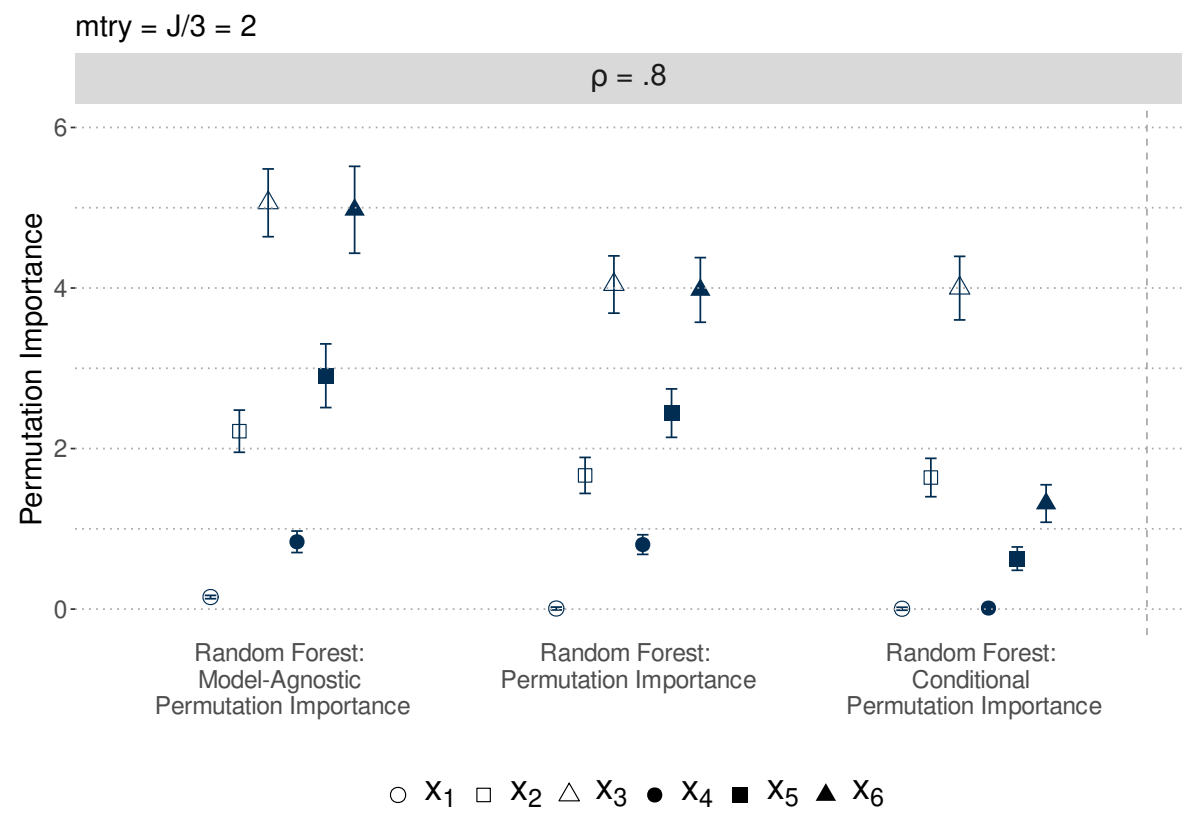

Figure 8: Mean ${ }^{+} /$- 1 standard deviation of permutation importance for three uncorrelated $\left(x_{1}, x_{2}\right.$, $x_{3}$; empty shapes) and three block-correlated $\left(x_{4}, x_{5}, x_{6}\right.$; solid shapes; $\left.\rho=.8\right)$ across $R=1000$ replications. In contrast to Figure 7 , mtry was not tuned, but set to the suggested value $\frac{J}{3}=2$.

For both settings of mtry, the conditional permutation importance measure ascribes much lower permutation importance in general, but particularly to the predictors that are correlated $\left(x_{5}\right.$ through $x_{6}$ ). The reason for this is that it permutes the predictor of interest conditionally on values of the correlated predictors, and this permutation within grids results in limited variability. Hence, if one wanted to compare permutation importance measures to parameters in standard parametric models, the original permutation importance measures behaves more similar to marginal measures (such as zero-order correlations), while the conditional permutation importance behaves more similar to partial measures (such as partial regression coefficients with their corresponding significance tests).

\section{Do the different machine learning methods give the same importance patterns?}

Looking at the results for the neural network in Figure 7, no major differences in the means between uncorrelated and correlated predictors can be detected, neither for the permutation importance nor for the relative weight importance measure. This is largely due to the simple architecture of the neural network with only one neuron in the hidden layer. This architecture allows the neural network to directly reflect the properties of the data generating process. This

\footnotetext{
${ }^{15}$ One may notice that for $x_{6}$, which is also among the block-correlated predictors, the unconditional importance is higher than that of its uncorrelated counterpart $x_{3}$ in Figure 7 (with mtry $=4$ ), but not in Figure 8 (with mtry $=2$ ). This is because this highly influential predictor has a high chance of being selected in the top split of many trees when mtry equals 4 , whereas when mtry equals $2, x_{6}$ will often not be part of the randomly pre-selected set of only two predictors available for the top split. As those predictors selcted in the top split can produce higher importance values, this in turn reduces the upward shift for $x_{6}$ we saw in Figure 7 .
} 
shows that the variable importance patterns do not only reflect properties of the data, but also properties of the machine learning method.

We do see, however, that the standard deviation of the variable importance measures across replications is higher for the block-correlated predictors (lower row of Figure 7). As the relative weight importance for the neural network is bounded at $0 \%$ and $100 \%$, floor and ceiling effects together with higher variation lead to a slightly higher average relative weight importance for $\left(x_{4}\right)$ and a slightly lower average relative weight importance for $x_{6}$ in the condition with block-correlations (lower row).

\section{Intermediate Conclusion}

In summary, we find that - particularly in the presence of correlations between predictors - the patterns drawn by the different variable importance measures differ from each other and from the pattern that the regression coefficients of a linear regression model would show. They also depend on which machine learning method (here random forest vs. neural network) and which tuning parameters have been used.

Of course, we only presented a selection of various variable importance measures, and for other model-specific and model-agnostic measures, the results may have had a different pattern.

However, the lessons learned from this section, such as that the absolute height of an importance measure should not be interpreted and that the pattern of importances can differ between machine learning methods, should be considered when using alternative variable importance techniques.

The reader may find the differences between unconditional and conditional variable importance measures particularly astonishing. Following the argumentation of Debeer and Strobl (2020), we would like to point out that there is no right or wrong here, but that it depends on the research question whether a more marginal or a more partial view may be of interest. The conditional variable importance aiming at reflecting partial effects succeeds in reducing the importance of correlated predictors with no true effect of their own - but at the cost of overreducing the importance of correlated predictors with true effects. At the same time, the unconditional importance is not fully marginal. With larger values of mtry, the effect of each predictor on top of those predictors that have already been included in the model is already assessed in the tree-building process, so that the unconditional importance behaves more similarly to the conditional importance with larger mtry (Strobl et al., 2008). Therefore, both types of permutation importance should be considered as intermediate rather than extreme points on the marginal-to-partial continuum (cf. Debeer \& Strobl, 2020).

We also want to assure the reader that here we have intentionally chosen an extreme simulation design, where the correlation structure is particularly pronounced, for illustrative purposes. In real data, the differences between the different types of variable importance measure will often be much less pronounced, and often the methods will agree upon the most important variables. The main take home message from this section should be that in cases where the importance measures rank predictors differently, this is likely due to correlations between predictors: Predictors whose importance is ranked higher by an unconditional and lower by a conditional variable importance measure have a smaller partial effect, but are correlated with other influential predictors. These variables can still be good predictors (shoe size in the marginal view), but may not add as much information on top of other predictors (shoe size on top of age in the partial view).

Last but not least, the notion of variable importance is built on the assumption that the true predictors are included in the machine learning method. In particular in datasets with a large number of predictors, different sets of predictors may be equally capable of predicting the outcome and thus receive high importance values. Particularly if the interest is to interpret predictor 
importance, a large number of observations in comparison to the number of predictors is necessary (see Efron, 2020).

\section{Part II: Detection of Interaction Effects}

One of the main advantages of machine learning methods in comparison to parametric models is that the machine learning method has the ability to automatically detect interaction effects and include them into the method's predictions. However, in addition to a black box prediction we are often also interested in learning which predictors are involved in interaction effects. For this purpose, interpretation techniques such as the two-dimensional PD and ALE plots and colored or centered ICE plots are useful tools to describe interaction effects visually, while the Hamilton interaction statistic can be used to describe interaction strength numerically. However, there are also situations where these interpretation techniques can be misleading. In this part, we illustrate how it can happen that one may find interaction effects that do not exist or miss interaction effects that do exist when using these interpretation techniques.

\section{Two-Dimensional PD and ALE Plots}

Many of the interpretation techniques presented in part I (e.g., PD plots, ALE plots, permutation importance) average across persons to demonstrate the effect of an individual predictor. In consequence, interaction effects, such as varying slopes between different groups of persons, as they would occur in the presence of an interaction effect, may cancel out. Hence, univariate methods like PD and ALE plots may be misleading when machine learning predictions contain higher-order interaction effects (Casalicchio et al., 2019; Goldstein et al., 2015; Greenwell et al., 2018; Molnar et al., 2020), which is the rule rather than the exception.

Examples of this phenomenon are shown in Figure 9 displaying PD (Panel A) and ALE curves (Panel B). We generated data including main and interaction effects between each of $x_{4}, x_{5}$, and $x_{6}$ with $x_{z}$ (see Equation 2)

$$
\begin{aligned}
y_{i}= & 5+0 \cdot x_{i 1}+2 \cdot x_{i 2}+3 \cdot x_{i 3}+0 \cdot x_{i 4}+2 \cdot x_{i 5}+3 \cdot x_{i 6}+ \\
& 3 \cdot x_{i 4} \cdot x_{z}+3 \cdot x_{i 5} \cdot x_{z}+3 \cdot x_{i 6} \cdot x_{z}+\varepsilon_{i}
\end{aligned}
$$

and fitted a random forest. We can see that the PD and ALE curves are a bit more "wiggly" for $x_{4}, x_{5}, x_{6}$, and $x_{z}$ than for the predictors without interaction effects in the data generating process $\left(x_{1}, x_{2}\right.$, and $\left.x_{3}\right)$. But, neither based on PD nor ALE curves, a systematic difference between the shape of the effects of $x_{1}$ and $x_{4}, x_{2}$ and $x_{5}$, or $x_{3}$ and $x_{6}$ on the outcome can be detected. Similarly, the curves of predictor $x_{z}$ that has strong interaction effects with $x_{4}, x_{5}$, and $x_{6}$ shows a relatively flat shape (plots for the neural network look similar and are shown in Figure E.1 in Appendix E). Hence, if we only looked at univariate PD or ALE plots, we would completely miss the interaction effects - that we know exist because here the data generating process is known, but might not suspect in an empirical application of these plots. In order to be able to detect two-way interaction effects, PD and ALE plots have been extended to bivariate plots showing main and interaction effects of two predictors jointly.

\section{Two-Dimensional PD Plots}

The computation of two-dimensional PD predictions is very similar to the computation of one-dimensional PD predictions that we presented above. The main difference is that now we 
(A) PD plots

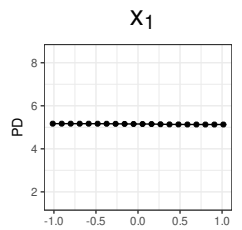

$\beta_{1}=0$

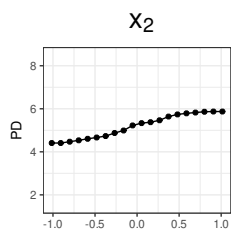

$\beta_{2}=2$

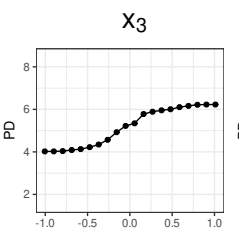

$\beta_{3}=3$

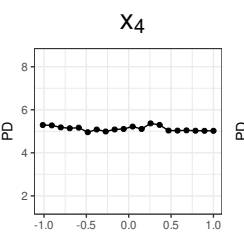

$\beta_{4}=0 ; \beta_{42}=3$

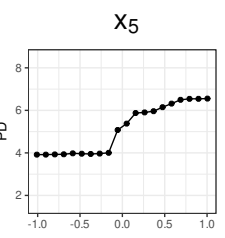

$\beta_{5}=2 ; \beta_{5 z}=3$

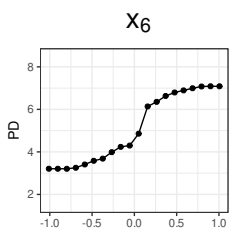

$\beta_{6}=3 ; \beta_{6 z}=3$

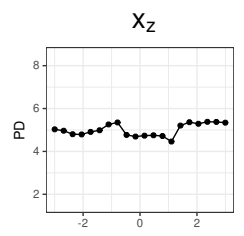

$\beta_{z}=0 ; \beta_{4 z}=\beta_{5 z}=\beta_{6 z}=3$

(B) ALE plots

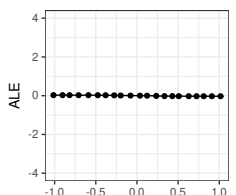

$\beta_{1}=0$

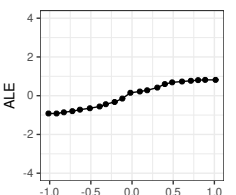

$\beta_{2}=2$

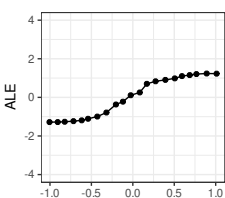

$\beta_{3}=3$

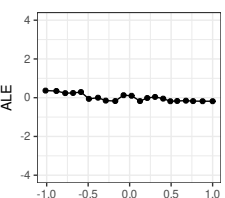

$\beta_{4}=0 ; \beta_{42}=3$

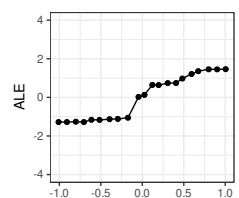

$\beta_{5}=2 ; \beta_{5 z}=3$

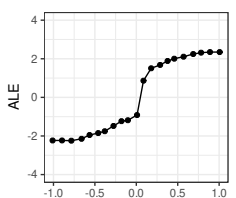

$\beta_{6}=3 ; \beta_{6 z}=3$

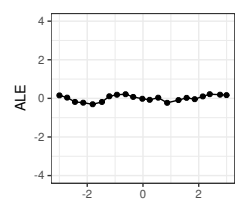

$\beta_{z}=0 ; \beta_{4 z}=\beta_{5 z}=\beta_{6 z}=3$

Figure 9: PD (Panel A) and ALE curves (Panel B) for data generated using the data generating process in Equation 2 fitted with the random forest. All visualizations in one row have fixed y-axis scales. Plots for the neural network are shown in Figure E.1 in Appendix E.

compute PD predictions over a grid of different positions $\boldsymbol{a}$ on predictor $x_{j}$ and different positions $\boldsymbol{b}$ on predictor $x_{j^{\prime}}$ by applying the following procedure:

1. Use the trained machine learning method to make a prediction $\hat{y}_{i}$ for each person $i$ for the positions $a$ and $b$ of predictors $x_{j}$ and $x_{j^{\prime}}$, using the observed values of person $i$ for all other predictors.

$$
\hat{y}_{i}=\hat{f}^{M L}\left(x_{i 1}, \ldots, x_{i(j-1)}, a, x_{i(j+1)}, \ldots, x_{i\left(j^{\prime}-1\right)}, b, x_{i\left(j^{\prime}+1\right)}, \ldots, x_{i J}\right)
$$

2. Average the predictions over all persons $i$ to obtain the PD prediction of the combination of $a$ and $b$.

$$
P D_{\left(j, j^{\prime}\right)}(a, b)=\frac{1}{N} \sum_{i=1}^{N} \hat{y}_{i}
$$

3. To obtain $P D_{\left(j, j^{\prime}\right)}$ predictions of predictors $x_{j}$ and $x_{j^{\prime}}$, repeat this procedure for all other combinations of $a$ and $b$.

The resulting plots are shown further below.

\section{Two-Dimensional ALE Plots}

While two-dimensional PD plots display main and interaction effects jointly, main effects are excluded in two-dimensional ALE plots (Apley \& Zhu, 2020) so that they only show interaction effects between the two predictors. We will see that due to this property, two-dimensional ALE plots may facilitate discovering interactions that are present in the data. Two-dimensional ALE are computed as follows:

1. The predictors $x_{j}$ and $x_{j^{\prime}}$ are split into $K_{j} \times K_{j}^{\prime}$ grid cells.

2. Predictions at each corner of the cell are made for each person $i$ located within the cell $\left(a_{j}^{(k-1)}<x_{i j}<a_{j}^{(k)}\right.$ and $\left.b_{j^{\prime}}^{\left(k^{\prime}-1\right)}<x_{i j^{\prime}}<b_{j^{\prime}}^{\left(k^{\prime}\right)}\right)$. Then, differences of the predictions at the cell corners are computed in the following way (see also Figure 10 for an illustration): 


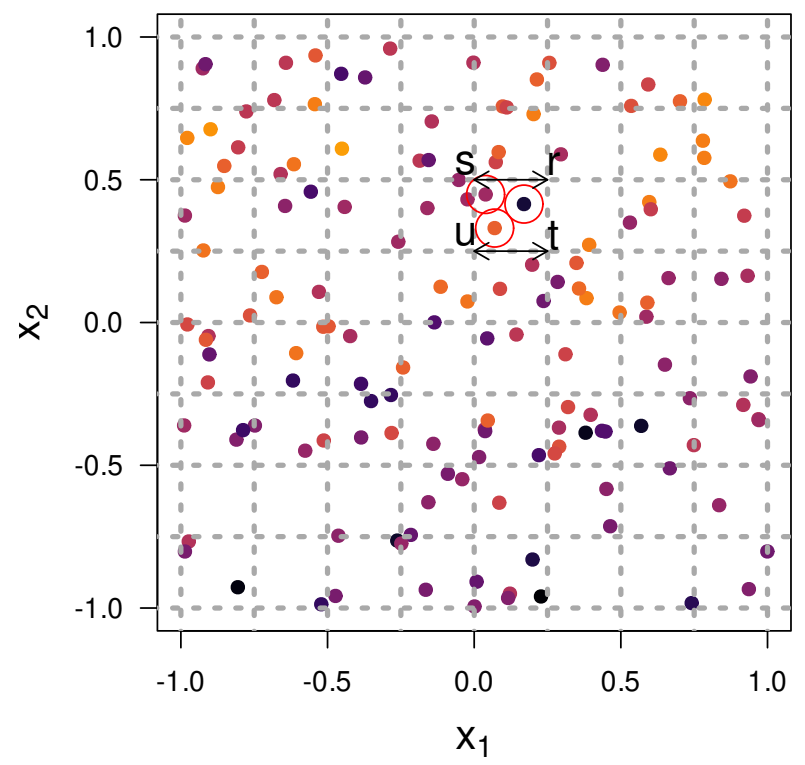

\section{- Color gradient of the \\ - outcome, with lighter colors \\ - reflecting higher values}

○ persons within a grid cell | grid cell boundary

-

Figure 10: Illustration of computation of two-dimensional Accumulated Local Effects (ALE). Predictions are made for each person $i$ located within the grid's cell at each corner of the cell and subtracted in the form $(r-s)-(t-u)$ with $r:\left(a_{j}^{(k)}, b_{j^{\prime}}^{\left(k^{\prime}\right)}\right), s:\left(a_{j}^{(k-1)}, b_{j^{\prime}}^{\left(k^{\prime}\right)}\right), t:\left(a_{j}^{(k)}, b_{j^{\prime}}^{\left(k^{\prime}-1\right)}\right)$, $u:\left(a_{j}^{(k-1)}, b_{j^{\prime}}^{\left(k^{\prime}-1\right)}\right)$.

$$
\begin{aligned}
\Delta_{i}\left(A_{j}^{(k)}, B_{j^{\prime}}^{\left(k^{\prime}\right)}\right) \\
=\left[\hat{f}^{M L}\left(x_{i 1}, \ldots, x_{i(j-1)}, a_{j}^{(k)}, x_{i(j+1)}, \ldots, x_{i\left(j^{\prime}-1\right)}, b_{j^{\prime}}^{\left(k^{\prime}\right)}, x_{i\left(j^{\prime}+1\right)}, \ldots, x_{i J}\right)\right. \\
\left.\quad-\hat{f}^{M L}\left(x_{i 1}, \ldots, x_{i(j-1)}, a_{j}^{(k-1)}, x_{i(j+1)}, \ldots, x_{i\left(j^{\prime}-1\right)}, b_{j^{\prime}}^{\left(k^{\prime}\right)}, x_{i\left(j^{\prime}+1\right)}, \ldots, x_{i J}\right)\right] \\
\quad-\left[\hat{f}^{M L}\left(x_{i 1}, \ldots, x_{i(j-1)}, a_{j}^{(k)}, x_{i(j+1)}, \ldots, x_{i\left(j^{\prime}-1\right)}, b_{j^{\prime}}^{\left(k^{\prime}-1\right)}, x_{i\left(j^{\prime}+1\right)}, \ldots, x_{i J}\right)\right. \\
\left.\quad-\hat{f}^{M L}\left(x_{i 1}, \ldots, x_{i(j-1)}, a_{j}^{(k-1)}, x_{i(j+1)}, \ldots, x_{i\left(j^{\prime}-1\right)}, b_{j^{\prime}}^{\left(k^{\prime}-1\right)}, x_{i\left(j^{\prime}+1\right)}, \ldots, x_{i J}\right)\right]
\end{aligned}
$$

3. As for one-dimensional ALE plots, the persons' differences in prediction are averaged across all $n^{\left(k, k^{\prime}\right)}$ persons located within each cell $\left(A_{j}^{(k)}, B_{j^{\prime}}^{\left(k^{\prime}\right)}\right)$

$$
\bar{\Delta}\left(A_{j}^{(k)}, B_{j^{\prime}}^{\left(k^{\prime}\right)}\right)=\frac{1}{n^{\left(k, k^{\prime}\right)}} \sum_{x_{i\left(j, j^{\prime}\right)} \in\left(A_{j}^{(k)}, B_{j^{\prime}}^{\left(k^{\prime}\right)}\right)} \Delta_{i}\left(A_{j}^{(k)}, B_{j^{\prime}}^{\left(k^{\prime}\right)}\right)
$$


4. The averaged prediction differences are accumulated up to the $k^{\text {th }}$ and $k^{\text {th }}$ cell.

$$
A L E^{\mathrm{unc}}\left(A_{j}^{(k)}, B_{j}^{\left(k^{\prime}\right)}\right)=\sum_{k^{*}=1}^{k} \sum_{k^{\prime *}=1}^{k^{\prime}} \bar{\Delta}\left(A_{j}^{\left(k^{*}\right)}, B_{j^{\prime}}^{\left(k^{\prime *}\right)}\right)
$$

5. ALE effects are "doubly-centered" (Apley \& Zhu, 2020, p.7) meaning that they have a mean of 0 similar to one-dimensional ALE effects, and in addition main effects of $x_{j}$ and $x_{j^{\prime}}$ are subtracted from overall ALE effects. In consequence, only interaction effects are reflected by two-dimensional ALE (Apley \& Zhu, 2020).

6. First, the main effects are subtracted so that only interaction effects remain:

$$
\begin{aligned}
A L E^{\mathrm{IA}}\left(A_{j}^{(k)}, B_{j^{\prime}}^{\left(k^{\prime}\right)}\right) & =A L E^{\mathrm{unc}}\left(A_{j}^{(k)}, B_{j^{\prime}}^{\left(k^{\prime}\right)}\right) \\
& -A L E^{\mathrm{unc}}\left(A_{j}^{(k)}\right)-A L E^{\mathrm{unc}}\left(B_{j^{\prime}}^{\left(k^{\prime}\right)}\right)
\end{aligned}
$$

7. Second, analogous to one-dimensional ALE effects, subtracting a constant $\left(C_{j j^{\prime}}\right)$ centers the second-order ALE effect around 0.

$$
A L E\left(A_{j}^{(k)}, B_{j^{\prime}}^{\left(k^{\prime}\right)}\right)=A L E^{\mathrm{IA}}\left(A_{j}^{(k)}, B_{j^{\prime}}^{\left(k^{\prime}\right)}\right)-C_{j j^{\prime}}
$$

with

$$
C_{j j^{\prime}}=\frac{1}{N} \sum_{k=1}^{K} \sum_{k^{\prime}=1}^{K^{\prime}} n^{\left(k, k^{\prime}\right)} A L E^{\mathrm{IA}}\left(A_{j}^{(k)}, B_{j^{\prime}}^{\left(k^{\prime}\right)}\right)
$$

\section{Interpretation of two-dimensional PD and ALE plots}

Figure 11 shows two-dimensional PD (Panel A) and ALE (Panel B) plots for data generated under Equation 2 and fitted with the random forest (plots for the neural network look similar and are shown in Figure E.2 in Appendix E). In the Figures, predictors $x_{1}$ through $x_{6}$ are placed on the $\mathrm{x}$-axis, while $x_{z}$ with which $x_{4}$ through $x_{6}$ interact is placed on the y-axis. The predicted value of the outcome (PD plot, Panel A) or the accumulated local effect (ALE plot; Panel B) is reflected by the color gradient, and the legend of the color gradient indicates the range of the effect in the plot.

In the first three columns, the true effects are main effects for $x_{2}$ and $x_{3}\left(\beta_{1}=0, \beta_{2}=2\right.$, $\beta_{3}=3$ ) and no interaction effects are present in the data generating process. In the last three columns, the true effects included the same main effects, and in addition the data generating process contained interaction effects of $x_{4}$ through $x_{6}$ with $x_{z}\left(\beta_{4 z}=3, \beta_{5 z}=3, \beta_{6 z}=3\right.$; see Equation 2). It is noteworthy that in our example, we know the data generating processes and expect to see interactions of $x_{4}$ through $x_{6}$ with $x_{z}$. When examining empirical data, we would have to assess several combinations of predictors or follow specific hypotheses to detect interaction effects.

\section{What is the difference between two-dimensional PD and ALE plots?}

Two-dimensional PD plots show main and interaction effects jointly (Panel A in Figure 11). Hence, in the first three columns we would expect to see no systematic pattern in the leftmost plot, and then an increasing difference between the left and the right side in the second and third plot reflecting the increasing main effects of $x_{2}$ and $x_{3}$. In the last three columns, we expect to see main and interaction effects together. Interaction effect show as coloring in opposite corners or in the diagonal of the plot. The interaction effect is best visible in the fourth column where $x_{4}$ interacts with $x_{z}$, but $x_{4}$ has no main effect. In the two rightmost columns, the main effects of $x_{5}$ 

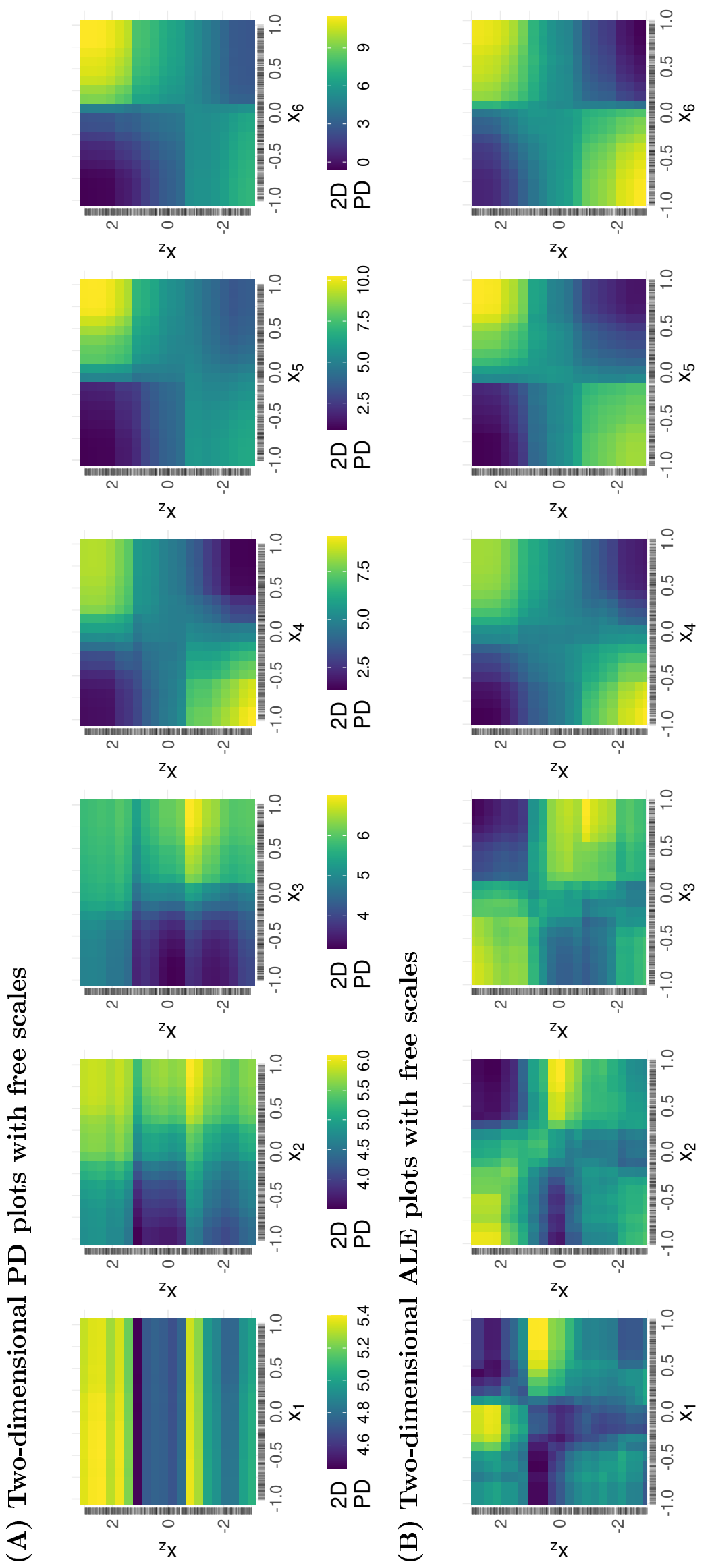

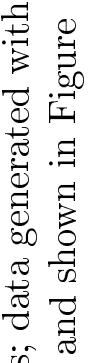

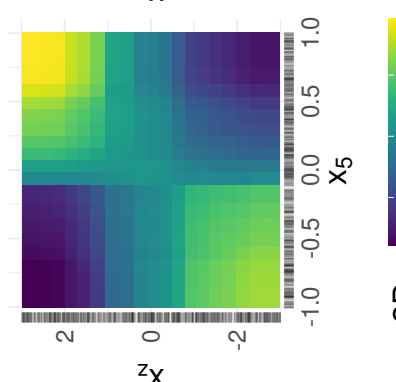

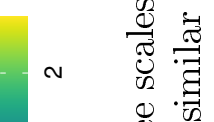

ष

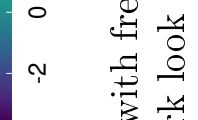

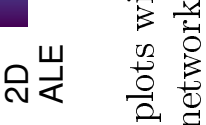

团

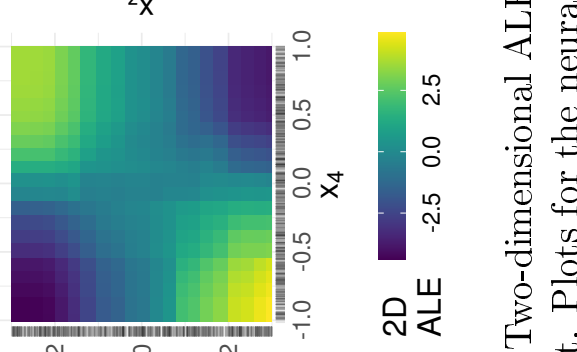

$1 \frac{0}{1}$

$\ddot{m}$

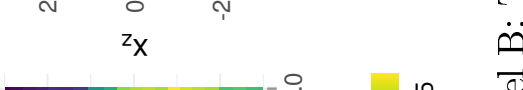

¿)

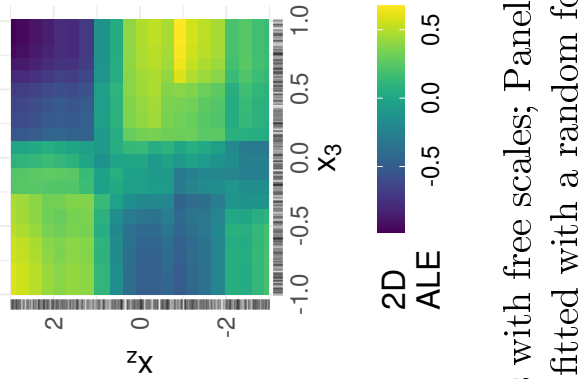

索㕝

ఖ

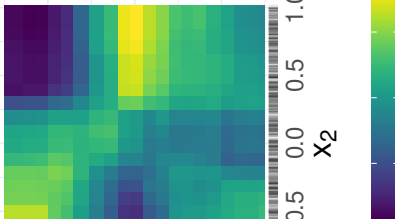

คิ

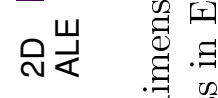
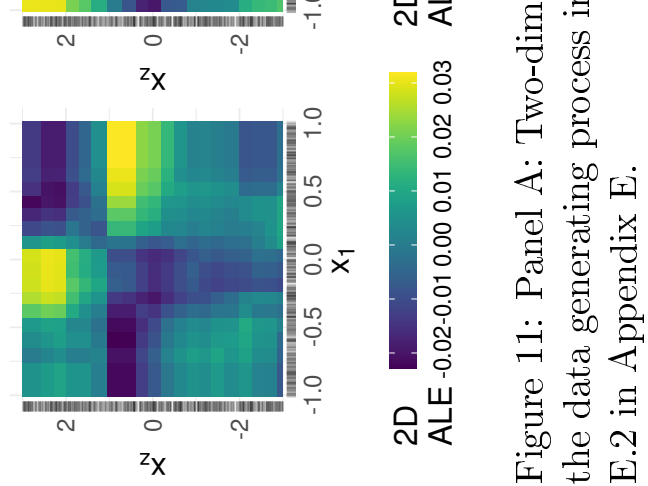
and $x_{6}$ are shown together with interaction effects of $x_{5}$ and $x_{6}$ with $x_{z}$, respectively. Therefore, the interaction effect is optically attenuated because the color gradient reflects both, main and interaction effects jointly.

In contrast, two-dimensional ALE plots show the interaction effects excluding the main effects (Panel B in Figure 11). Hence, when only main effects are present in the data generating process (the first three columns), we expect to see no effects or a noisy pattern. In the last three columns, we expect to see only the interaction effects. Compared to the two-dimensional PD plots in Panel A, one may find the interaction effect easier to spot in last three columns of Panel B, as interaction effects are displayed in isolation in two-dimensional ALE plots.

\section{Is there a risk to over-interpret these plots?}

The two-dimensional PD and ALE plots are heatmaps, where the values of the outcome are reflected by a color gradient. Typically, the most extreme colors (here dark blue versus light yellow) are set to the minimum and maximum by default. Of course, the minimum and maximum will very likely differ between each plot as the displayed effects vary in size (e.g., between the first and fourth column for ALE). Ignoring the scale of the color gradient, researchers looking for effects in their data might be mislead to assuming a much larger effect than there actually is.

To demonstrate this effect, Figure 12 shows the two-dimensional PD and ALE plots with scales fixed to the minimum and maximum across all plots. Fixing the scales requires to know the scale's minimum and maximum when creating the visualizations. In practice, researchers will create one visualization at a time with free scales, determine the scale's minimum and maximum, and then have to recreate all visualizations with fixed scales.

Here, we do know the true data generating process. However, when using empirical data it will be much more difficult to detect and interpret main and interaction effects. In addition, more complex data generating processes including, for instance, higher-order (e.g., three-way) interactions may additionally lead to more complex presentations of effects in interpretation techniques that would require to use an interaction statistics of higher order or to compare multiple two-dimensional plots. The reason why we are using simulated data here for illustrative purposes was the possibility to compare different sizes and types of effects within the same method, such as plots without interaction effects (first three columns in Panel B) with plots with interaction effects (last three columns in Panel B), which is not possible using empirical data where the true effects are unknown. In order to faciliate direct and explicit comparisons between visualizations of different types in empirical settings, we review a suggestion for the visual inference method further below.

\section{Colored and Centered ICE Plots}

As ICE plots display the effect of a predictor for each person, they can also be used to visualize bivariate interaction effects. However, when the sample size is large and many ICE curves are displayed, varying slopes are difficult to distinguish. Figure 13 shows ICE plots for the data generating process in Equation 2 with main effects for $x_{1}$ to $x_{3}$ and main and interaction effects for $x_{4}$ to $x_{6}$ with $x_{z}$. In the first three columns, we can see that the ICE curves are parallel, while in the last four columns, we see that the ICE curves cross. Even though we have drawn a random sample of 200 persons and increased the transparency for ICE curves (inspired by Goldstein et al., 2015), it remains difficult to distinguish the single ICE curves 


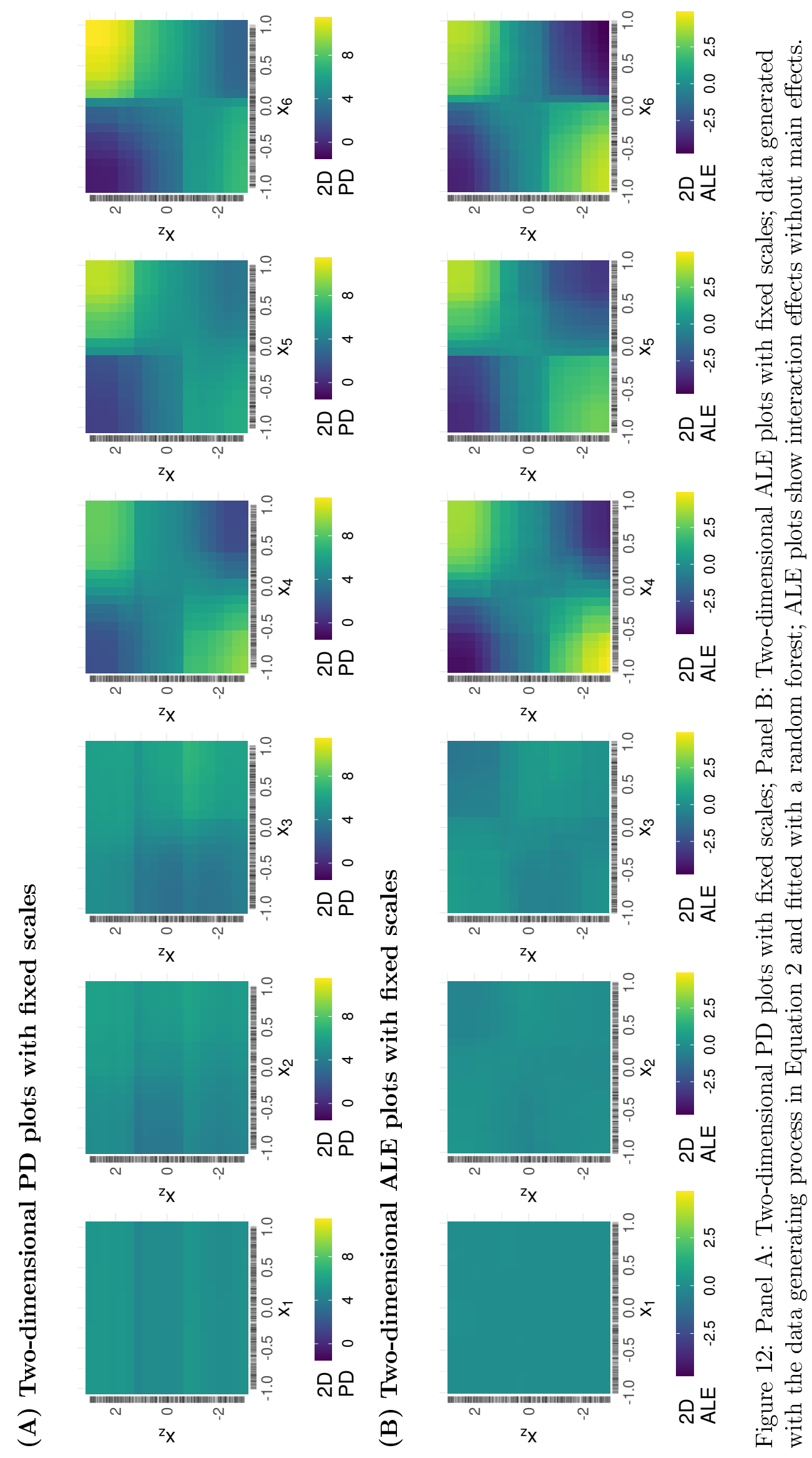




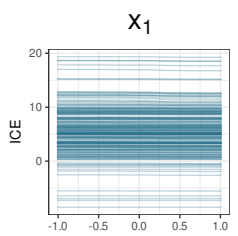

$\beta_{1}=0$

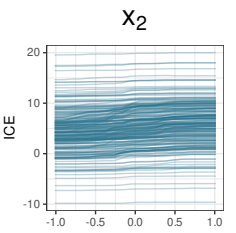

$\beta_{2}=2$

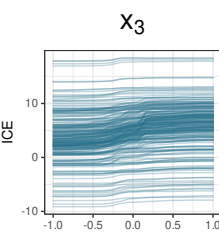

$\beta_{3}=3$

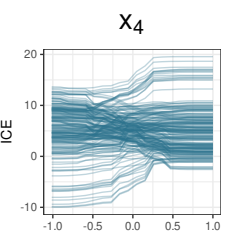

$\beta_{4}=0 ; \beta_{42}=3$

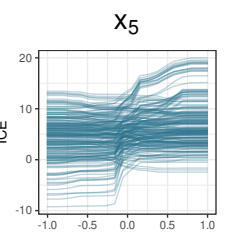

$\beta_{5}=2 ; \beta_{5 z}=3$

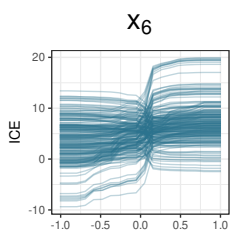

$\beta_{6}=3 ; \beta_{6 z}=3$

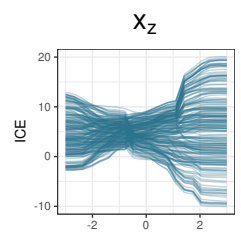

$\beta_{z}=0 ; \beta_{4 z}=\beta_{5 z}=\beta_{6 z}=3$

Figure 13: ICE curves for a random sample of 200 persons based on data generated using the data generating process in Equation 2 for the random forest.

\section{How can interactions be visualized with ICE plots?}

To further facilitate interpretability, ICE plots can be colored by another predictor that is suspected to interact with the predictor of interest (see Panel A in Figure 14). This principle is well known from interpreting interactions in linear regression, where we see the effect of $x_{j}$ in the different slopes for the different groups created by $x_{z}$. Coloring ICE curves makes it easier to distinguish where an interaction with $x_{z}$ does not occur (first three columns in Panel A in Figure 14) or where an interaction is present (last three columns in Panel A in Figure 14). In the first three columns, the curves are parallel and not detectably sorted by the color gradient based on $x_{z}$, which indicates that no interaction effects are present. In the last three columns, on the contrary, the slopes of the predictors $x_{4}$ to $x_{6}$ depend on $x_{z}$. The slopes are structured systematically by color as the light blue slopes are positive, while the dark blue slopes are less steep or even negative.

In order to make interactions even more detectable, Goldstein et al. (2015) proposed to center ICE curves vertically (see Panel B of Figure 14) by letting the ICE curves of all persons originate at a predicted value of 0 . This is achieved by subtracting each person's predicted value at the minimum of $x_{j}$ from their ICE predictions. Hence, centered ICE curves visualize the model's predictions relative to a reference location $a^{\text {ref }}$ (here the minimum, $a^{\text {ref }}=x_{j}^{\text {min }}$, but also the maximum or other values for $a^{\text {ref }}$ would be possible):

$$
I C E_{i j}^{\text {centered }}(a)=I C E_{i j}(a)-I C E_{i j}\left(a^{\text {ref }}\right) .
$$

The centered ICE curves (Panel B of Figure 14) facilitate to see whether the slopes of predictors $x_{4}$ through $x_{6}$ vary as a function of $x_{z}$. For instance, in the last three columns, the light blue slopes are positive, while the dark blue slopes are less steep or even negative. Note however that as a consequence of vertical centering neither absolute values of the predicted outcome nor any differences between persons can be interpreted (Goldstein et al., 2015). In Appendix B, we further illustrate the effect of centering on ICE curves for different reference locations. Note again, that we know the data generating process which is why we colored the ICE curves by $x_{z}$. In empirical data, we would need to try out more combinations of predictors or follow a hypothesis-based procedure.

\section{Interaction Statistic}

Besides the graphical technique for interaction detection, Friedman and Popescu (2008) proposed an interaction statistic that describes two-way interactions between predictors $x_{j}$ and $x_{j^{\prime}}$ and overall interaction strength numerically. The interaction statistic is based on the idea that an interaction between two predictors $x_{j}$ and $x_{j^{\prime}}$ occurs when the effect of $x_{j}$ on the outcome depends on the value of $x_{j^{\prime}}$, and the prediction cannot be explained by the main effects of each of the two 

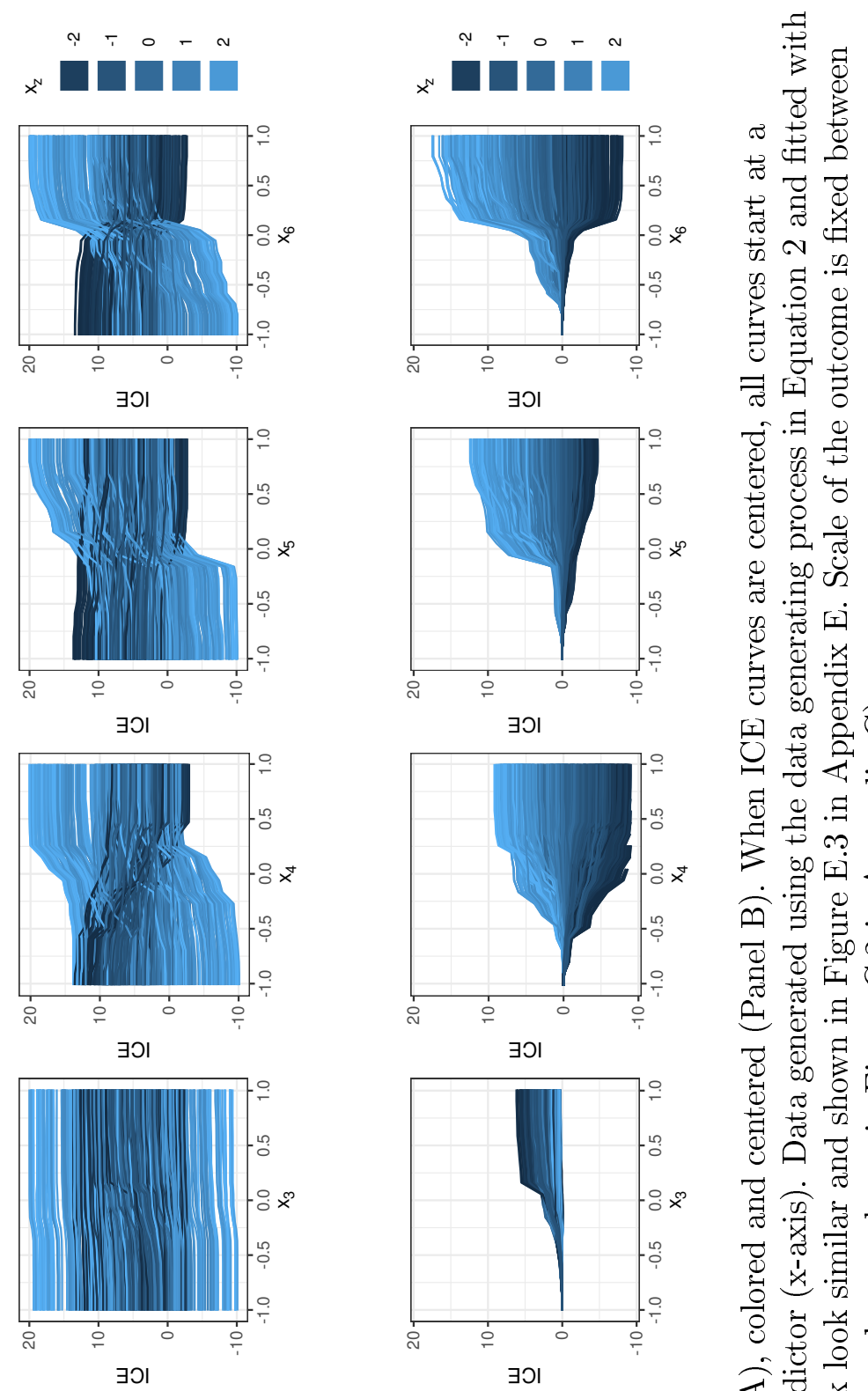

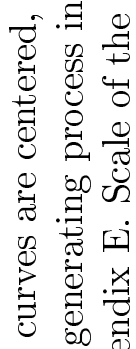

ن 0 क

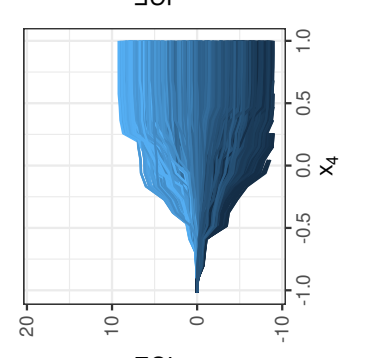

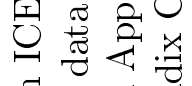

웡. 뭉

皮界 $\dot{\hat{\oplus}} \cdot \because \Xi$

จ

คี

$\Xi \Xi$

卷 80

焉焉

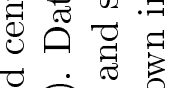

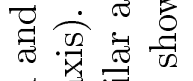

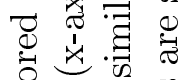

일

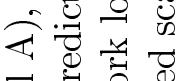

党范总

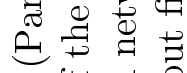

एक

\& 莺

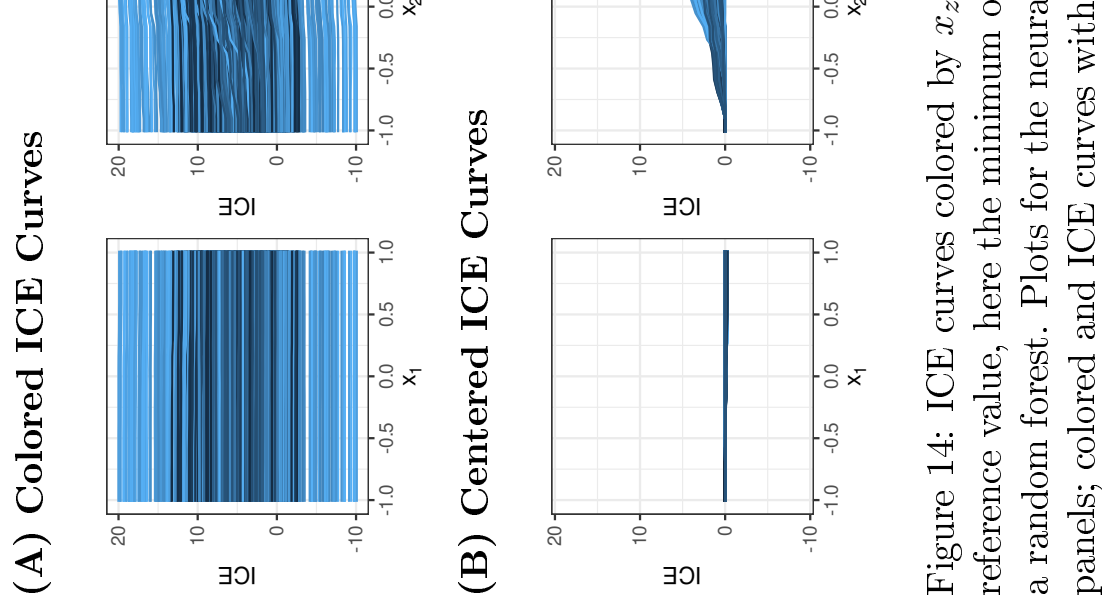


predictors alone.

\section{Two-way interaction statistic}

The two-way interaction statistic for $x_{j}$ and $x_{j^{\prime}}$ can be computed as follows:

1. From the two-dimensional PD predictions of two predictors representing their main and interaction effects, $P D_{\left(j, j^{\prime}\right)}\left(x_{i j}, x_{i j^{\prime}}\right)$, subtract each of the uni-dimensional PD predictions representing the two main effects, $P D_{j}\left(x_{i j}\right)$ and $P D_{j^{\prime}}\left(x_{i j^{\prime}}\right)$, for each person $i$. The difference is what cannot be explained by main effects. Hence the two-way interaction between $x_{j}$ and $x_{j}^{\prime}$ is defined as:

$$
\begin{aligned}
\Delta_{i\left(j, j^{\prime}\right)}^{(I A)} & =P D_{\left(j, j^{\prime}\right)}\left(x_{i j}, x_{i j^{\prime}}\right) \\
& -P D_{j}\left(x_{i j}\right) \\
& -P D_{j^{\prime}}\left(x_{i j^{\prime}}\right)
\end{aligned}
$$

Please note that each component, $P D_{\left(j, j^{\prime}\right)}\left(x_{i j}, x_{i j^{\prime}}\right), P D_{j}\left(x_{i j}\right)$, and $P D_{j^{\prime}}\left(x_{i j^{\prime}}\right)$, is centered so that its mean across persons is 0 .

2. The difference is squared and summed over all persons $i$. The squared sum is divided by the squared sum of the centered two-way PD predictions for the main and interaction effects of the two predictors $P D_{\left(j, j^{\prime}\right)}\left(x_{i j}, x_{i j^{\prime}}\right)$ to set the value range of the interaction statistic between 0 and 1 .

$$
H_{\left(j, j^{\prime}\right)}^{2}=\frac{\sum_{i=1}^{N}\left(\Delta_{i\left(j, j^{\prime}\right)}^{(I A)}\right)^{2}}{\sum_{i=1}^{N} P D_{\left(j, j^{\prime}\right)}\left(x_{i j}, x_{i j^{\prime}}\right)^{2}}
$$

3. By taking the square root, the two-way interaction statistic is obtained:

$$
H_{\left(j, j^{\prime}\right)}=\sqrt{H_{\left(j, j^{\prime}\right)}^{2}}
$$

Hence, the two-way interaction statistic measures the fraction of variance of the two-dimensional PD predictions that is not captured by the PD predictions for main effects. By using a divide-by-total approach, the range of the statistic is set between 0 and 1 . When the statistic is 1 , it means that the two-way PD predictions are fully composed of an interaction between $x_{j}$ and $x_{j^{\prime}}$. When the statistic is 0 , it means that the prediction can be completely decomposed into the main effects of $x_{j}$ and $x_{j^{\prime}}$, and that no interaction between $x_{j}$ and $x_{j^{\prime}}$ has contributed to the two-way PD predictions.

\section{Overall interaction statistic}

Similarly, the overall interaction statistic of the predictor $x_{j}$ with any other predictor can be defined as:

1. From the machine learning method's complete prediction for person $i$, $\hat{f}^{M L}\left(x_{i 1}, \ldots, x_{i j}, \ldots, x_{i J}\right)$, subtract the univariate PD prediction reflecting the main effect of $x_{j}$ of person $i, P D_{j}\left(x_{i j}\right)$, and the PD predictions reflecting main and interaction effects of all predictors except $x_{j}$ of person $i, P D_{(1, \ldots, j-1, j+1, \ldots, J)}\left(x_{i 1}, \ldots, x_{i(j-1)}, x_{i(j+1)}, \ldots, x_{i J}\right)$. The resulting difference is the component of $\hat{f}^{M L}\left(x_{i 1}, \ldots, x_{i j}, \ldots, x_{i J}\right)$ that is due to interaction effects that $x_{j}$ is involved in. Note that in order to quantify overall interaction statistic, the 
machine learning method's complete predictions $\hat{f}^{M L}$, that contain all possible interactions, are used (whereas in the two-way formula, the two-way $P D$ predictions are used).

$$
\begin{aligned}
\Delta_{i j}^{(\mathrm{IA})} & =\hat{f}^{M L}\left(x_{i 1}, \ldots, x_{i j}, \ldots, x_{i J}\right) \\
& -P D_{j}\left(x_{i j}\right) \\
& -P D_{(1, \ldots, j-1, j+1, \ldots, J)}\left(x_{i 1}, \ldots, x_{i(j-1)}, x_{i(j+1)}, \ldots, x_{i J}\right)
\end{aligned}
$$

Again each component, $\hat{f}^{M L}\left(x_{i 1}, \ldots, x_{i j}, \ldots, x_{i J}\right), P D_{j}\left(x_{i j}\right)$ and $P D_{(1, \ldots, j-1, j+1, \ldots, J)}\left(x_{i 1}, \ldots, x_{i(j-1)}, x_{i(j+1)}, \ldots, x_{i J}\right)$, is centered so that its mean across persons is 0 .

2. The difference is squared and summed over all persons $i$. One then divides the squared sum by the squared sum of the centered machine learning method prediction in order to set the value range of the interaction statistic between 0 and 1 .

$$
H_{j}^{2}=\frac{\sum_{i=1}^{N}\left(\Delta_{i j}^{(\mathrm{IA})}\right)^{2}}{\sum_{i=1}^{N}\left(\hat{f}^{M L}\left(x_{i 1}, \ldots, x_{i j}, \ldots, x_{i J}\right)\right)^{2}}
$$

3. Take the square root to obtain the interaction statistic:

$$
H_{j}=\sqrt{H_{j}^{2}}
$$

While setting the range of the statistic between 0 and 1 may facilitate the interpretation of the magnitude of the interaction effect, nonetheless there is no significance test to decide whether the statistic is large enough to suggest an interaction effect. Beyond that, the scaling procedure comes with the risk that in the absence of main effects, random noise appears as interaction effects as we will illustrate in the following section (Friedman \& Popescu, 2008).

To further illustrate this phenomenon, we generated data using the data generating process in Equation 2, including main effects and interaction effects ${ }^{16}$. As before, we set the sample size to $N=1000$ persons, generated $R=1000$ datasets, fitted a random forest with mtry $=7$ and a neural network with 9 neurons in the hidden layer and a regularization of 0.05 based on the pilot study, and calculated the overall and two-way interaction statistic using the R package iml.

\section{How can we judge whether a two-way interaction statistic $>0$ is meaningful?}

Panel A in Figure 15 shows the two-way interaction statistic between the predictors $x_{1}$ through $x_{6}$ and $x_{z}$ for the random forest (upper row) and the neural network (lower row across $R=1000$ replications. For instance, in the left upper panel, we see the two-way interaction statistic of predictor $x_{1}$ with all other predictors (on the x-axis from left to right: $x_{2}$ through $x_{6}$, and $x_{z}$ ). Given the data generating model, we expect to see two-way interactions between $x_{4}, x_{5}$, and $x_{6}$ with $x_{z}$ (effects located at the right side of the vertical dashed line in the panels for $x_{4}$ to $x_{6}$ ), but no indication of interactions for all other combinations of predictors. The expected interaction effects of $x_{4}, x_{5}$, and $x_{6}$ with $x_{z}$ are clearly visible.

However, for the random forest, we find small two-way interaction effects for predictors with main effects (e.g., $x_{2}$ and $x_{3}$ with $x_{z}$ ). This is because, random forests are designed to detect interaction effects rather than main effects via multiple splits. As a consequence, main effects might be misunderstood as interactions. At the same time, for the neural network, the interaction

\footnotetext{
${ }^{16}$ as a reminder: $y_{i}=5+0 \cdot x_{i 1}+2 \cdot x_{i 2}+3 \cdot x_{i 3}+0 \cdot x_{i 4}+2 \cdot x_{i 5}+3 \cdot x_{i 6}+3 \cdot x_{i 4} \cdot x_{z}+3 \cdot x_{i 5} \cdot x_{z}+3 \cdot x_{i 6} \cdot x_{z}+\varepsilon_{i}$
} 
(A) Two-way Interaction Statistic

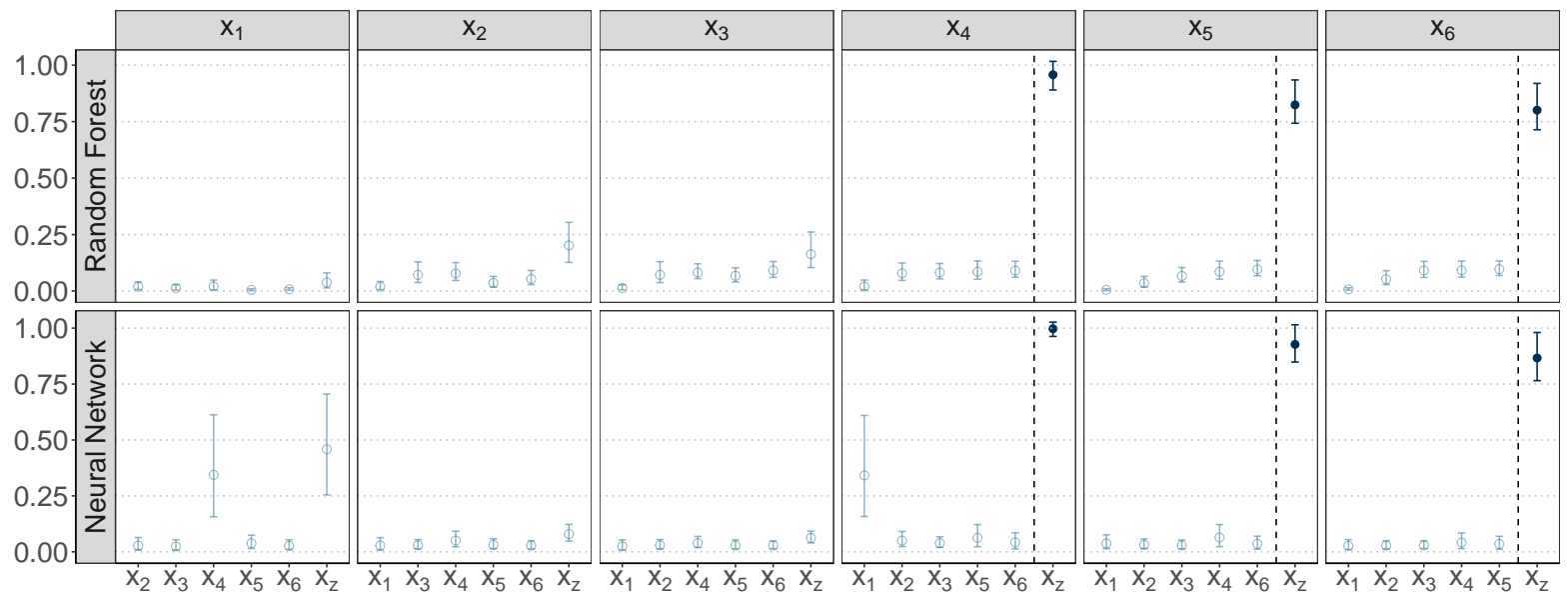

(B) Overall Interaction Statistic

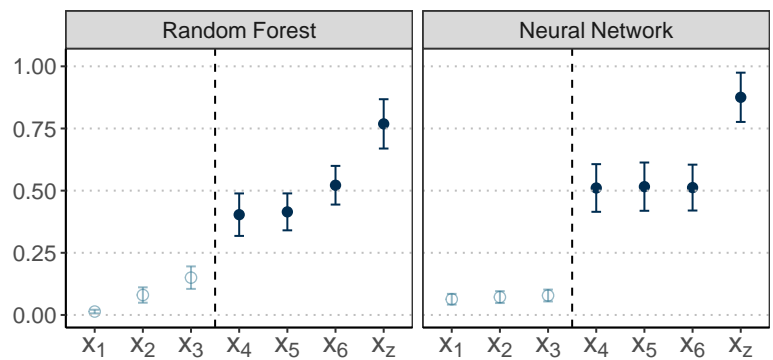

Figure 15: Mean +/_ 1 standard deviation of two-way interaction statistic (Panel A) and the overall interaction statistic (Panel B) for data generated with interaction effects of $x_{4}, x_{5}, x_{6}$, with $x_{z}$ (see Equation 2) across $R=1000$ replications. Expected interactions are located at the right side of the vertical dashed line.

statistic falsely suggests interactions between $x_{1}$ and $x_{4}$ or, as well as $x_{1}$ and $x_{z}$ (though there is large variation between simulation replications). This is because $x_{1}, x_{4}$, and $x_{z}$ have no main effects in the data generating model $\left(x_{1}, x_{4}\right.$, and $\left.x_{z}\right)$. Friedman and Popescu (2008) stated that "only those predictors that are deemed globally relevant [...] need be considered for interaction effects. This is often a small subset of all predictors" (p. 935, Friedman \& Popescu, 2008). In line with that, Greenwell et al. (2018) observed that the interaction statistic did not reflect the true interaction effects of simulated data, but suggested interaction effects for predictors with null main effects. Hence, our results suggest that the two-way interaction statistic should be interpreted with caution when variable importance is low (see also Friedman \& Popescu, 2008).

\section{Does an overall interaction statistic $>0$ signal the presence of an interaction?}

Panel B in Figure 15 shows the overall interaction statistic across $R=1000$ replications. As the data generating process contained interaction effects of $x_{4}, x_{5}$, and $x_{6}$ with $x_{z}$, we expect to see higher values for the interaction statistic on the right-hand side of the vertical dashed line. Indeed, we see that the predictor $x_{z}$ has large interaction statistic, followed by $x_{4}, x_{5}$, and $x_{6}$, while $x_{1}$, $x_{2}$, and $x_{3}$, that only had main effects in the data generating process, yield smaller values of the interaction statistic as expected. 
However, we see that despite that the interaction statistic is scaled from 0 to 1 , it is not straightforward to interpret its absolute height. For instance, the overall interaction statistic of $x_{z}$ does not reach 1 even though $x_{z}$ only had interaction effects in the data generating process. For the random forests, the overall interaction statistic for $x_{6}$, for instance, is slightly larger than the interaction statistic for $x_{4}$ and $x_{5}$ despite the same size of the interaction effect in the data generating process. Again, this might due the random forest misinterpreting main effects (in particular for $x_{6}$ ) for interaction effects.

In case that we had empirical data with an unknown data generating process similar to Equation 2, and would observe patterns of the interaction statistic as in Figure 15, we would correctly assume that $x_{4}, x_{5}, x_{6}$, and $x_{z}$ have high interaction strength. Based on the two-way interaction statistic and a fitted neural network, however, we might additionally, and erroneously, suspect $x_{1}$ and $x_{4}$, as well as $x_{1}$ and $x_{z}$ to also interact with each other. By using the interaction statistic, researchers can gain information which variables might interact with each other. So the interaction statistic can be used to identify potential candidates for interaction effects, even though its absolute height should be interpreted with caution. These interaction candidates can then further be evaluated using two-dimensional PD or ALE plots and colored or centered ICE curves as shown above.

\section{Visual Inference}

As pointed out several times throughout this paper, here we had the advantage of knowing the data generating process when we interpreted the different visualization methods for machine learning methods. However, in reality, knowledge about the data generating process has to be acquired in the data analysis process and is not available beforehand. In consequence, it is usually much more difficult to detect the importance and shape of main and interaction effects by means of interpretation techniques, but also to distinguish these results from patterns that have arisen by chance. The concept of visual inference (e.g., Chowdhury et al., 2015; Few, 2009; Majumder et al., 2010; Wickham et al., 2010) can support researchers working with empirical data to make direct comparisons based on visual interpretation techniques. We will briefly describe the idea behind visual inference and then illustrate the method by further examining an interaction effect detected in a two-dimensional ALE plot using empirical data provided by Lawrie et al. (2020).

\section{Idea and Procedure}

Visual inference is based on the idea that humans are very good at detecting patterns, but very bad at dealing with probabilities and chance. Therefore, humans may believe that they see patterns where these appear at random, a phenomenon that is called apophenia (Few, 2009; Wickham et al., 2010). In consequence, there is a risk that patterns are overinterpreted, in particular in exploratory analyses with visualization tools, such as interpretation techniques for machine learning methods. Visual inference, however, can make such implicit comparisons ("that looks different than expected") explicit, and can therefore help to avoid false-positive conclusions (e.g., Chowdhury et al., 2015; Majumder et al., 2010).

One way of implementing statistical visual inference is an optical lineup, similar to a police lineup or identity parade: a "suspect" (the true visualization from the empirical data) and $M-1$ "fillers" (visualizations generated artificially under a null model) are presented. The fillers are created, for example, by permuting one of the predictors involved in the interaction so that the interaction effect vanishes (similar to the permutation importance principle). Then, the true visualization has to be identified from the lineup. In case that it can be clearly distinguished from 
the filler visualizations, the interaction effect is more pronounced than what we should expect to see by chance. The fillers show the spectrum of the effect under the null hypothesis, which helps us to evaluate whether the effect seen in the true visualization is more pronounced than the effect in the $M-1$ filler visualizations.

\section{Illustration Using Empirical Data}

In order to illustrate how the visual inference technique can be used to compare visualizations for machine learning methods, we briefly demonstrate its application using data of Study 2 by Lawrie et al. (2020) that we retrieved from $\mathrm{OSF}^{17}$. Inter alia, the authors analyzed main and interaction effects of the predictor variables culture (Romanian versus US) and age on subjective well-being using a multiple regression model. To mirror the analysis, we assessed the influence of several predictors (age, culture, gender, education, social class, high- and low-control coping) on subjective well-being via a conditional random forest $(\mathrm{mtry}=2)$ and visualized the interaction between age and culture in a two-dimensional ALE plot.

To assess whether the presumably visible interaction effect arose by chance, we applied the visual inference technique and created a visual lineup by applying the following procedure:

Step 1: We permuted the predictor culture (but permuting age would also be possible) in the dataset

Step 2: We fit a machine learning method (here a random forest)

Step 3: We created a filler visualization (here a two-dimensional ALE plot)

We repeated steps 1-3 until we created 19 filler visualization and then presented the true visualization together with the fillers to several of our colleagues. To make the ALE visualizations comparable, we set the scale limits of the color gradient to the minimum and maximum of the true plot for all visualizations (see Panel A of Figure 16). Here, the true visualization (second row, second column) has been identified with high confidence by our colleagues (and we presume the readers too), suggesting that the interaction effect that we see in the original data is truly different from a pattern that could have appeared by chance. Similar to statistical inference, the false positive rate can be reduced by increasing the number of filler visualizations, and the confidence in detecting a true effect with the visual inference technique increases with the number of raters, and the confidence with which they give their response. Again, we would like to highlight the importance of fixing the scale limits of the color gradient in visualizations of interpretation techniques. For this purpose, Panel B in Figure 16 shows the same visualizations as Panel A but without fixing the scale limits of the color gradient so that the scale of the color gradient reflects each visualization's individual span of ALE values. In consequence, small ALE effects appear more visually striking, making it much more difficult to identify the true visualization from the fillers.

Initially proposed in the area of computational statistics and engineering, we believe that the visual inference technique can be a very helpful tool also in psychological research. In combination with machine learning and visualization techniques, visual inference may support researchers in gauging whether a visually apparent effect may have occurred only by chance.

\section{Discussion}

Machine learning methods are black boxes that are very good at predicting the outcome, but require additional techniques to interpret how they have come to this prediction. We have shown that interpretation techniques for machine learning methods have great potential in demonstrating

${ }^{17}$ https://osf.io/sd265/ 
(A)
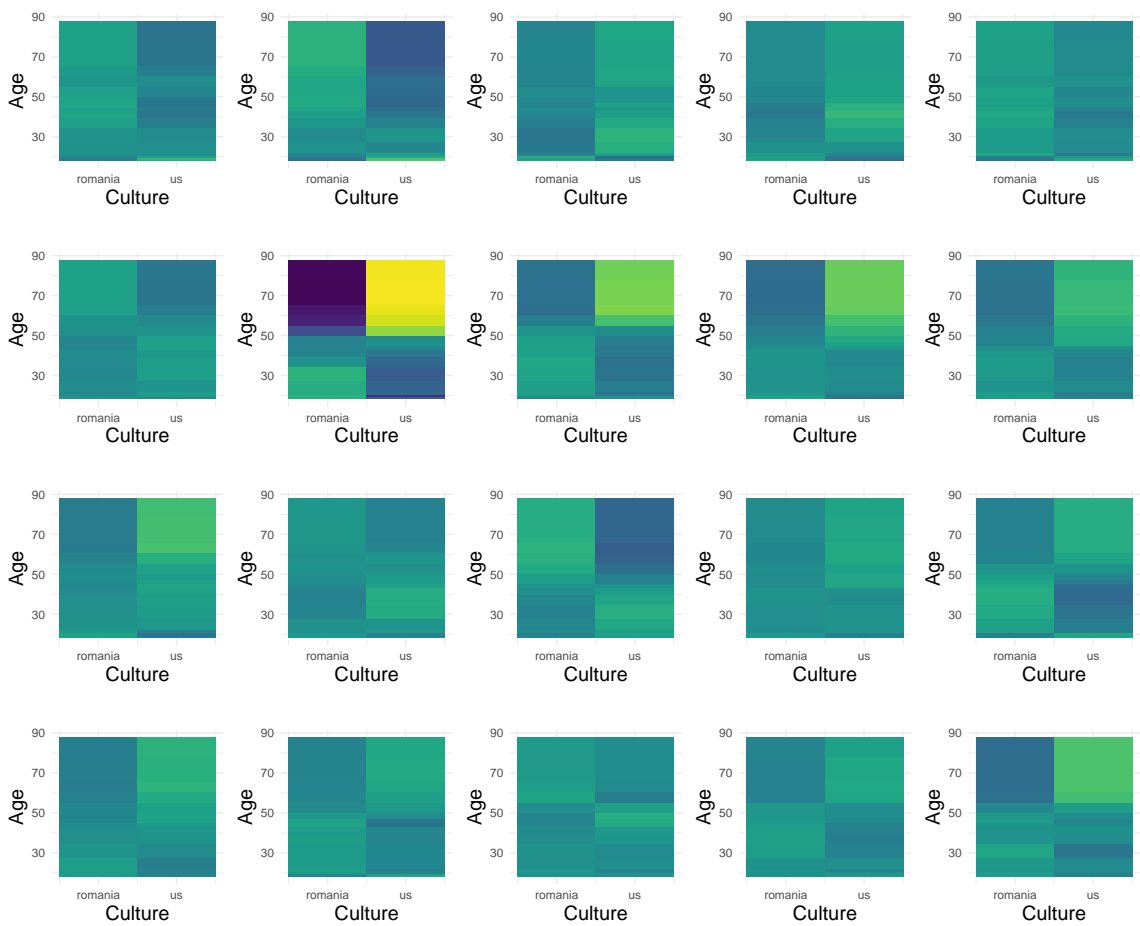

(B)
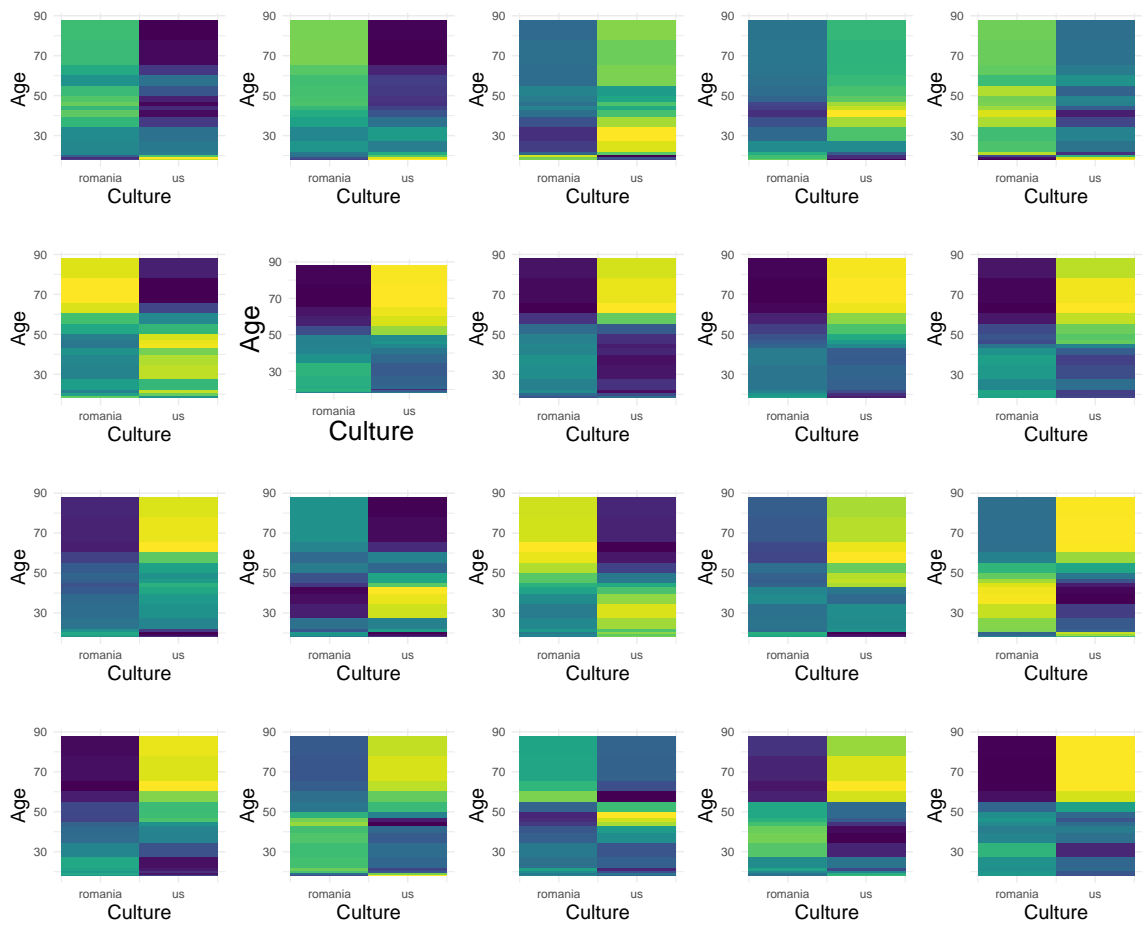

Figure 16: Two examples of visual inference lineups for two-dimensional Accumulated Local Effects (ALE) plots for the random forest; Panel A: scale for ALE fixed in all plots to the minimum and maximum of the true plot; Panel B: no scale fixed for ALE. 
which predictors have been relevant to predict the outcome, how the shape of the underlying effect looks like and how interaction effects can be detected. At the same time, these interpretation techniques entail a high risk for misinterpretation. In this article, we focused on the potential pitfalls with regard to correlated predictors and interaction detection for global interpretation techniques.

\section{What interpretation techniques (don't) do}

The information gained from PD, ICE, and ALE plots as well as variable importance differs depending on whether we used a random forest or a neural network. Hence, even though these interpretation techniques are model-agnostic and can be applied to a diverse range of machine learning methods, their results may differ as a function of the machine learning method itself, in particular when the machine learning methods differ in their underlying mechanisms and inherent assumptions (see also Štrumbelj \& Kononenko, 2014).

Interpretation techniques often only provide a single value of the measure, but do not indicate sampling uncertainty (e.g., Molnar et al., 2020). To show the uncertainty across different datasets, here we used replicated data simulations when we showed the analyses for the variable importance and interaction statistic measures. This enabled us to quantify how stable the measures are across $R=1000$ replications, but this would not be possible in practice. Hence, in contrast to statistical models, and unlike the accuracy of prediction that is usually evaluated in the machine learning framework, uncertainty in interpretation techniques are typically not quantified when these are applied to empirical data.

Interpretation techniques are tools that help us grasp the patterns that the black box has learned. As is the case when using parametric models for observational data, interpretation techniques do not provide us with information about the true causal relationship or the data generating process itself, but about the prediction function of the machine learning method (see e.g., Moraffah et al., 2020; Turner Lee, 2018).

We have focused on global interpretation techniques that aim at describing, for example, the overall importance for the entire sample. Of course, for very complex prediction function, interpretation becomes more difficult, and a global measure of relevance or shape might not be sufficient to convey a holistic picture of the predictor effect. Pursuing this argument, local interpretation techniques, such as Local Interpretable Explanations (LIME; Ribeiro et al., 2016) or Shapley values (Štrumbelj \& Kononenko, 2011, 2014) have been proposed. These local methods aim at describing predictions at certain locations in the predictor space or for individual persons. Since predictions of machine learning methods can vary largely at different locations or for different persons, local techniques have quickly gained popularity and we are currently preparing a discussion of their characteristics and limitations, in particular in psychological research.

Another important point is that, for instance, a PD plot shows the marginal effect of different values of a predictor on the outcome in a machine learning method. But it does not tell us how the predicted value of a specific person would have changed if that person had had another value of the predictor. Hence, interpretation techniques cannot describe how changing a predictor or (components of) the model would have changed the prediction, as such conclusions would require experimental data (see e.g., Moraffah et al., 2020; Mothilal et al., 2020).

\section{What researchers should pay attention to}

Besides the points raised in the main text, we would like to draw the reader's attention to a few more aspects that should be kept in mind: 
Independent of which data and which machine learning method is chosen for a specific analysis, it is sensible to assess and evaluate prediction accuracy before proceeding with interpretation techniques. In this regard, Breiman (2002b) stated that "the better the model fits the data, the more sound the inferences about the black box are" (p. 4). Hence, evaluating and optimizing model fit by careful tuning is a necessary precondition for interpreting machine learning predictions (see also Debeer \& Strobl, 2020; Molnar et al., 2020).

The reasoning behind this argument is that we cannot learn about associations between predictors and the outcome from a model with a bad fit. At the same time, a good fit does not necessarily mean that the predictors have a true effect on the outcome (e.g., shoe size that predicts reading ability; see also Efron, 2020; Shmueli, 2010, for discussions). Particularly in settings with a large number of predictors, different groups of predictors can constitute good predictions on their own. In typical psychological research settings, pre-selecting predictors based on previous studies can be beneficial in this regard. Overall, while good model fit does not mean that the model is good, a model with a bad fit should not be used as the basis for interpretation.

In line with that, some researchers question the gains in predictive accuracy or model fit of machine learning methods compared to more interpretable models, such as single decision trees, regression models or generalized additive models (see e.g., Christodoulou et al., 2019; Fokkema et al., 2022; Hand, 2006; Holte, 1993). In particular for high-stakes decision where it is crucial to be able to communicate the reasons for the decision, classical parametric models might be an appropriate choice. Hence, psychological researchers may want to carefully evaluate whether a black box method is the right tool for their research question before the analysis (see Efron, 2020; Rudin, 2019, for discussions).

As outlined at various locations in the article, using visualizations as a basis for interpretation of machine learning results, researchers use many degrees of freedom with regard to their design. Fixing the y-axis or color scale between plots (as in Figure 5, 12, or 14) may result in substantially different impression regarding the size of effects than freeing the y-axis (Figure 11, C.1, or C.2). Hence researchers should be aware that such design decisions can easily influence the conclusion drawn from the analysis.

It is also important to take the characteristics of the interpretation technique itself into account. As an example, PD and non-centered ICE plots display the marginal predicted values and are therefore on the same scale as the outcome. In contrast, ALE plots display the difference in prediction and centered ICE plots display the prediction relative to a reference position and hence, do not give any information about the absolute value of the outcome.

Finally, besides evaluating whether the outcome increases over the range of the predictor per se, one should always reflect on whether this change in the outcome is substantially meaningful for the psychological research question.

\section{Conclusion}

As machine learning and interpretation techniques have become an integral parts of many research fields, knowing about and applying these methods expands the toolbox of research in the psychological science. Additionally combining interpretation techniques with visualization tools, such as the visual inference method, and with a data analysis process where a parametric model that is applied on new data follows an initial exploratory analysis based on machine learning and interpretation techniques, may be a fruitful way in exploring and describing complex psychological processes. 


\section{References}

Apley, D. W., \& Zhu, J. (2020). Visualizing the effects of predictor variables in black box supervised learning models. Journal of the Royal Statistical Society: Series B (Statistical Methodology), 82, 1059-1086. https://doi.org/10.1111/rssb.12377

Azen, R., \& Budescu, D. V. (2003). The dominance analysis approach for comparing predictors in multiple regression. Psychological Methods, 8, 129-148. https://doi.org/10.1037/1082-989X.8.2.129

Bates, C. J., \& Jacobs, R. A. (2020). Efficient data compression in perception and perceptual memory. Psychological Review, 127, 891-917. https://doi.org/10.1037/rev0000197

Breiman, L., Friedman, J., Olshen, R., \& Stone., C. (1984). Classification and Regression Trees. Boca Raton, Chapman; Hall/CRC.

Breiman, L. (2001a). Random forests. Machine Learning, 45, 5-32. https://link.springer.com/content/pdf/10.1023/A:1010933404324.pdf

Breiman, L. (2001b). Statistical modeling: The two cultures. Statistical Science, 16, 199-231. https://doi.org/10.1214/ss/1009213726

Breiman, L. (2002a). Manual on setting up, using, and understanding random forests V3.1. https://www.stat.berkeley.edu/ breiman/Using_random_forests_V3.1.pdf

Breiman, L. (2002b). Wald Lecture II: Looking inside the black box. https://www.stat.berkeley.edu/ breiman/wald2002-2.pdf

Breiman, L., \& Cutler, A. (2002). Random Forest: RRforest Fortran code. Retrieved January 28, 2022, from https://www.stat.berkeley.edu/ breiman/RandomForests/reg_software.htm

Casalicchio, G., Molnar, C., \& Bischl, B. (2019). Visualizing the feature importance for black box models. Machine Learning and Knowledge Discovery in Databases, 655-670. https://doi.org/10.1007/978-3-030-10925-7_40

Chowdhury, N. R., Cook, D., Hofmann, H., Majumder, M., Lee, E.-K., \& Toth, A. L. (2015). Using visual statistical inference to better understand random class separations in high dimension, low sample size data. Computational Statistics, 30, 293-316. https://doi.org/10.1007/s00180-014-0534-x

Christodoulou, E., Ma, J., Collins, G. S., Steyerberg, E. W., Verbakel, J. Y., \& Van Calster, B. (2019). A systematic review shows no performance benefit of machine learning over logistic regression for clinical prediction models. Journal of Clinical Epidemiology, 110, 12-22. https://doi.org/10.1016/j.jclinepi.2019.02.004

Debeer, D., \& Strobl, C. (2020). Conditional permutation importance revisited. BMC Bioinformatics, 21, 1-30. https://doi.org/10.1186/s12859-020-03622-2

Efron, B. (2020). Prediction, estimation, and attribution. International Statistical Review, 88, 28-59. https://doi.org/10.1111/insr.12409

Eid, M., Gollwitzer, M., \& Schmidt, M. (2015). Statistik und Forschungsmethoden (4. Edition). Weinheim, Basel, Beltz Verlag.

Few, S. (2009). Now you see it: Simple visualization techniques for quantitative analysis (1st Edition). Analytics Press. 
Fisher, A., Rudin, C., \& Dominici, F. (2019). All models are wrong, but many are useful: Learning a variable's importance by studying an entire class of prediction models simultaneously. Journal of Machine Learning Research, 20, 1-81. http://jmlr.org/papers/v20/18-760.html

Fokkema, M., Iliescu, D., Greiff, S., \& Ziegler, M. (2022). Machine learning and prediction in psychological assessment. European Journal of Psychological Assessment, 38, 165-175. 10.1027/1015-5759/a000714

Fokkema, M., \& Strobl, C. (2020). Fitting prediction rule ensembles to psychological research data: An introduction and tutorial. Psychological Methods, 25, 636-652. https://doi.org/10.1037/met0000256

Friedman, J. H. (2001). Greedy function approximation: A gradient boosting machine. The Annals of Statistics, 29, 1189-1232. https://www.jstor.org/stable/2699986

Friedman, J. H., \& Popescu, B. E. (2008). Predictive learning via rule ensembles. Annals of Applied Statistics, 2, 916-954. https://doi.org/10.1214/07-AOAS148

Gevrey, M., Dimopoulos, I., \& Lek, S. (2003). Review and comparison of methods to study the contribution of variables in artificial neural network models. Ecological Modelling, 160, 249-264. https://doi.org/10.1016/S0304-3800(02)00257-0

Goldstein, A., Kapelner, A., Bleich, J., \& Pitkin, E. (2015). Peeking inside the black box: Visualizing statistical learning with plots of individual conditional expectation. Journal of Computational and Graphical Statistics, 24, 44-65. https://doi.org/10.1080/10618600.2014.907095

Gonzalez, O. (2021). Psychometric and machine learning approaches for diagnostic assessment and tests of individual classification. Psychological Methods, 26, 236-254. https://doi.org/10.1037/met0000317

Goretzko, D., \& Bühner, M. (2020). One model to rule them all? Using machine learning algorithms to determine the number of factors in explanatory factor analysis. Psychological Methods, 25, 776-786. https://doi.org/10.1037/met0000262

Greenwell, B. M., Boehmke, B. C., \& McCarthy, A. J. (2018). A simple and effective model-based variable importance measure, 1-27. http://arxiv.org/abs/1805.04755

Grömping, U. (2015). Variable importance in regression models. Wiley Interdisciplinary Reviews: Computational Statistics, 7, 137-152. https://doi.org/10.1002/wics.1346

Hand, D. J. (2006). Classifier technology and the illusion of progress. Statistical Science, 21, 1-14. https://doi.org/10.1214/088342306000000060

Hastie, T., Tibshirani, R., \& Jerome Friedman. (2008). The Elements of Statistical Learning: Data Mining, Inference, and Prediction (2nd Edition). New York, Springer Series in Statistics. https://books.google.de/books?id=tVIjmNS3Ob8C

Heaton, J. (2008). Feedforward Neural Networks. In K. Smith (Ed.), Introduction to Neural Networks with Java (2nd Edition, pp. 143-172). Heaton Research, Inc.

Henninger, M., Debelak, R., Rothacher, Y., \& Strobl, C. (2022). Interpretable machine learning for psychological research: Opportunities and pitfalls. Preprint. https://psyarxiv.com/xe83y 
Henninger, M., Debelek, R., Rothacher, Y., \& Strobl, C. (2022). Interpretable machine learning for psychological research: Opportunities and pitfalls. Supplementary Material. https://osf.io/zgc9w/

Holte, R. C. (1993). Very simple classification rules perform well on most commonly used datasets. Machine Learning, 11, 63-91.

Hong, M., Jacobucci, R., \& Lubke, G. (2020). Deductive data mining. Psychological Methods, 25, 691-707. https://doi.org/10.1037/met0000252

Hooker, G. (2004). Diagnostics and extrapolation in machine learning (Doctoral dissertation). Stanford University. https://rb.gy/io5tbm

Hooker, G., Mentch, L., \& Zhou, S. (2021). Please stop permuting features: Variable importance requires at least one more model, or there is no free variable importance. http://arxiv.org/abs/1905.03151

Hornik, K., Stinchcombe, M., \& White, H. (1989). Multilayer feedforward networks are universal approximators. Neural Networks, 2, 359-366. https://doi.org/10.1016/0893-6080(89)90020-8

Hothorn, T., Hornik, K., \& Zeileis, A. (2006). Unbiased recursive partitioning: A conditional inference framework. Journal of Computational and Graphical Statistics, 15, 651-674. https://doi.org/10.1198/106186006X133933

Hothorn, T., Hornik, K., \& Zeileis, A. (2015). ctree: Conditional Inference Trees. The Comprehensive $R$ Archive Network, 8, 1-34. https://rdrr.io/rforge/partykit/f/inst/doc/ctree.pdf

Hothorn, T., \& Zeileis, A. (2015). partykit: A modular toolkit for recursive partitioning in R. Journal of Machine Learning Research, 16, 3905-3909. http://jmlr.org/papers/v16/hothorn15a.html

Kim, H., \& Loh, W. (2001). Classification trees with unbiased multiway splits. Journal of the American Statistical Association, 96, 589-604. https://doi.org/10.1198/016214501753168271

Kuhn, M. (2020). caret: Classification and Regression Training. $R$ package version 6.0-86. https://cran.r-project.org/package $=$ caret

Lapuschkin, S., Wäldchen, S., Binder, A., Montavon, G., Samek, W., \& Müller, K. R. (2019). Unmasking Clever Hans predictors and assessing what machines really learn. Nature Communications, 10, 1-8. https://doi.org/10.1038/s41467-019-08987-4

Lawrie, S. I., Eom, K., Moza, D., Gavreliuc, A., \& Kim, H. S. (2020). Cultural variability in the association between age and well-being: The role of uncertainty avoidance. Psychological Science, 31, 51-64. https://doi.org/10.1177/0956797619887348

Liaw, A., \& Wiener, M. (2002). Classification and regression by randomForest. R News, 2, 18-22. https://cogns.northwestern.edu/cbmg/LiawAndWiener2002.pdf

Majumder, M., Hofmann, H., \& Cook, D. (2010). Visual statistical inference for regression parameters, 1-6. http://citeseerx.ist.psu.edu/viewdoc/download?doi=10.1.1.233. $2392 \&$ rep $=$ rep1\&type $=$ pdf 
Molnar, C. (2019). Interpretable Machine Learning: A Guide for Making Black Box Models Explainable. https://christophm.github.io/interpretable-ml-book

Molnar, C., Bischl, B., \& Casalicchio, G. (2018). iml: An R package for Interpretable Machine Learning. Journal of Open Source Software, 3, 786.

https://doi.org/10.21105/joss.00786

Molnar, C., König, G., Herbinger, J., Freiesleben, T., Dandl, S., Scholbeck, C. A., Casalicchio, G., Grosse-Wentrup, M., \& Bischl, B. (2020). General pitfalls of model-agnostic interpretation methods for machine learning models, (1049). https://arxiv.org/pdf/2007.04131.pdf

Moraffah, R., Karami, M., Guo, R., Raglin, A., \& Liu, H. (2020). Causal interpretability for machine learning: Problems, methods and evaluation. ACM SIGKDD Explorations Newsletter, 22, 18-33. https://doi.org/10.1145/3400051.3400058

Mothilal, R. K., Sharma, A., \& Tan, C. (2020). Explaining machine learning classifiers through diverse counterfactual explanations. Proceedings of the 2020 Conference on Fairness, Accountability, and Transparency, 607-617. https://doi.org/10.1145/3351095.3372850

Nicodemus, K. K., \& Malley, J. D. (2009). Predictor correlation impacts machine learning algorithms: Implications for genomic studies. Bioinformatics, 25, 1884-1890. https://doi.org/10.1093/bioinformatics/btp331

Nicodemus, K. K., Malley, J. D., Strobl, C., \& Ziegler, A. (2010). The behaviour of random forest permutation-based variable importance measures under predictor correlation. BMC Bioinformatics, 11, 1-13. https://doi.org/10.1186/1471-2105-11-110

Olden, J. D., Joy, M. K., \& Death, R. G. (2004). An accurate comparison of methods for quantifying variable importance in artificial neural networks using simulated data. Ecological Modelling, 178, 389-397. https://doi.org/10.1016/j.ecolmodel.2004.03.013

Philipp, M., Rusch, T., Hornik, K., \& Strobl, C. (2018). Measuring the stability of results from supervised statistical learning. Journal of Computational and Graphical Statistics, 27, 685-700. https://doi.org/10.1080/10618600.2018.1473779

Probst, P., Wright, M. N., \& Boulesteix, A. L. (2019). Hyperparameters and tuning strategies for random forest. Wiley Interdisciplinary Reviews: Data Mining and Knowledge Discovery, 9, 1-15. https://doi.org/10.1002/widm.1301

Ribeiro, M. T., Singh, S., \& Guestrin, C. (2016). 'Why should I trust you?' Explaining the predictions of any classifier, In Kdd '16: Proceedings of the 22nd ACM SIGKDD International Conference on Knowledge Discovery and Data Mining. https://doi.org/10.1145/2939672.2939778

Rothacher, Y., \& Strobl, C. (2022). Identifying informative predictor variables with random forests. Manuscript submitted for publication.

Rudin, C. (2019). Stop explaining black box machine learning models for high stakes decisions and use interpretable models instead. Nature Machine Intelligence, 1(5), 206-215. https://doi.org/10.1038/s42256-019-0048-x 
Schmidt-Hieber, J. (2020). Nonparametric regression using deep neural networks with ReLU activation function. Annals of Statistics, 48, 1875-1897. https://doi.org/10.1214/19-AOS1875

Scholbeck, C. A. (2018). Interpretierbares Machine-Learning. Post-hoc modellagnostische Verfahren zur Bestimmung von Prädiktoreffekten in Supervised-Learning-Modellen. Ludwig-Maximilians-Universität München Fakultät, Fakultät für Mathematik, Informatik und Statistik. https://epub.ub.uni-muenchen.de/60504/1/MA_Scholbeck.pdf

Segal, M. R. (2004). Machine learning benchmarks and random forest regression. Center for Bioinformatics and Molecular Biostatistics, 15. https://escholarship.org/uc/item/35x3v9t4

Shmueli, G. (2010). To explain or to predict? Statistical Science, 25(3), 289-310. https://doi.org/10.1214/10-STS330

Strasser, H., \& Weber, C. (1999). On the asymptotic theory of permutation statistics. Report Series SFB "Adaptive Information Systems and Modelling in Economics and Management Science", 8, 220-250. https://epub.wu.ac.at/102/

Strobl, C., Boulesteix, A., \& Augustin, T. (2006). Unbiased split selection for classification trees based on the Gini index. Computational Statistics 85 Data Analysis, 52, 483-501. https://doi.org/10.1016/j.csda.2006.12.030

Strobl, C., Boulesteix, A. L., Kneib, T., Augustin, T., \& Zeileis, A. (2008). Conditional variable importance for random forests. BMC Bioinformatics, 9, 1-11. https://doi.org/10.1186/1471-2105-9-307

Strobl, C., Boulesteix, A. L., Zeileis, A., \& Hothorn, T. (2007). Bias in random forest variable importance measures: Illustrations, sources and a solution. $B M C$ Bioinformatics, 8. https://doi.org/10.1186/1471-2105-8-25

Strobl, C., Hothorn, T., \& Zeileis, A. (2009). Party on! A new, conditional variable importance measure available in the party package. The $R$ Journal, (2), 14-17. https://epub.ub.uni-muenchen.de/9387/1/techreport.pdf

Strobl, C., Malley, J., \& Tutz, G. (2009). An introduction to recursive partitioning: Rationale, application, and characteristics of classification and regression trees, bagging, and random forests. Psychological Methods, 14, 323-348. https://doi.org/10.1037/a0016973

Štrumbelj, E., \& Kononenko, I. (2011). A general method for visualizing and explaining black-box regression models, In International Conference on Adaptive and Natural Computing Algorithms, Berlin, Heidelberg, Springer.

Štrumbelj, E., \& Kononenko, I. (2014). Explaining prediction models and individual predictions with feature contributions. Knowledge and Information Systems, 41, 647-665. https://doi.org/10.1007/s10115-013-0679-x

Turner Lee, N. (2018). Detecting racial bias in algorithms and machine learning. Journal of Information, Communication and Ethics in Society, 16, 252-260.

https://doi.org/10.1108/JICES-06-2018-0056 
Venables, W. N., \& Ripley, B. D. (2002). Modern Applied Statistics with S. Springer. http://www.stats.ox.ac.uk/pub/MASS4

Weidman, A. C., Sun, J., Vazire, S., Quoidbach, J., Ungar, L. H., \& Dunn, E. W. (2019). (Not) hearing happiness: Predicting fluctuations in happy mood from acoustic cues using machine learning. Emotion, 20, 642-658.

https://doi.org/10.1037/emo0000571

Wickham, H., Cook, D., Hofmann, H., \& Buja, A. (2010). Graphical inference for infovis. IEEE Transactions on Visualization and Computer Graphics, 16, 973-979. https://doi.org/10.1109/TVCG.2010.161

Zhao, Q., \& Hastie, T. (2019). Causal interpretation of black-box models. Journal of Business and Economic Statistics, 272-281. https://doi.org/10.1080/07350015.2019.1624293 


\section{A Glossary: Joint Notation of Interpretation Techniques}

Indices of variables, persons, and intervals

\begin{tabular}{ll}
\hline$i$ & person $i$ with $i \in(1, \ldots, N)$ \\
$i^{\prime}$ & another person \\
$j$ & predictor variable $j$ with $j \in(1, \ldots, J)$ \\
$j^{\prime}$ & another predictor variable \\
$t$ & tree $t$ with $t \in(1, \ldots$, nTree $)$ \\
$h$ & neuron $h$ in the hidden layer with $h \in(1, \ldots, H)$ \\
$k$ & interval $k$ with $k \in(1, \ldots K)$ \\
\hline$N$ & number of persons in the sample \\
$J$ & number of predictors \\
$H$ & number of neurons in the hidden layer \\
$O$ & number of neurons in the output layer \\
$n^{(k)}$ & number of persons located in the $k^{t h}$ interval \\
Predictor and Outcome Variables \\
\hline$y_{i}$ & observed value of person $i$ on the outcome \\
$\hat{y}_{i}$ & predicted value of person $i$ on the outcome \\
$x_{i j}$ & value of person $i$ on predictor $x_{j}$ \\
$x_{i(j-1)}$ & value of person $i$ on the predictor preceding $x_{j}$ \\
$x_{i(j+1)}$ & value of person $i$ on the predictor following $x_{j}$ \\
$x_{i^{\prime} j}$ & values of another person $i^{\prime}$ on the predictor $x_{j}$ \\
$\boldsymbol{x}_{z}$ & covariates to be conditioned on \\
\hline$a$ & some position or value \\
$b$ & some position or value
\end{tabular}

Other Definitions

\begin{tabular}{ll}
\hline$A_{j}^{(1)}, \ldots, A_{j}^{\left(K_{j}\right)}$ & $K$ intervals \\
{$\left[a_{j}^{(k-1)}, a_{j}^{(k)}\right]$} & value of the upper/lower boundary of the $k^{\text {th }}$ interval \\
\hline$\hat{f}^{M L}$ & $\begin{array}{l}\text { prediction function of the machine learning method } \\
\hat{\varepsilon}\end{array}$ \\
residual/prediction error
\end{tabular}




\section{B Graphical Illustrations of Equations}

To help the interested reader gain a thorough understanding of how the interpretation techniques are computed step by step, we illustrate the equations in a graphical manner here. We use the same notation as in the main text (see Appendix A for a glossary), but in addition use a color-coding to indicate which part of the equation and algorithm refers to which part in the visualization of the interpretation technique.

\section{B.1 Partial Dependence (PD)}

To compute PD predictions for a predictor $x_{j}$ to draw a PD curve:

1. Use the trained machine learning method to make a prediction $\hat{y}_{i}$ for each person $i$ for the position $a$ on predictor $x_{j}$, using the observed values of person $i$ for all other predictors.

$$
\hat{y}_{i}=\hat{f}^{M L}\left(x_{i 1}, \ldots, x_{i(j-1)}, \mathrm{a}, x_{i(j+1)}, \ldots, x_{i J}\right)
$$

2. Average the predictions over all persons $i$ to obtain the PD prediction of the point $a$.

$$
\mathbb{P D}_{j}(\mathrm{a})=\frac{1}{N} \sum_{i=1}^{N} \hat{y}_{i}
$$

3. To obtain $P D_{j}$ over the entire range of predictor $x_{j}$, repeat this procedure for all other positions a and draw the curve ${ }^{18}$.

\section{Partial Dependence Plot}

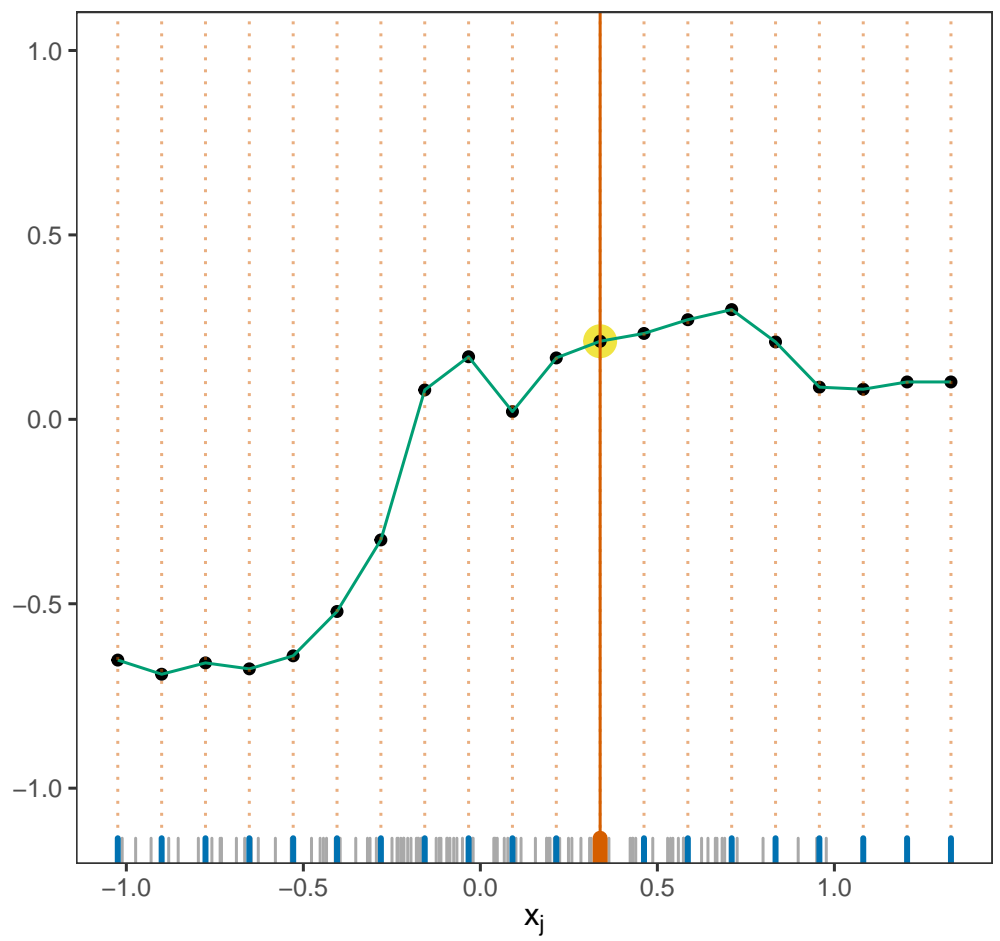

\footnotetext{
${ }^{18}$ Here some equally spaced positions $a$ based on $x_{j}$ are selected to draw the PD curve (default in the $R$ package iml).
} 


\section{B.2 Individual Conditional Expectations (ICE)}

ICE curves can be computed the same way as a PD curve, without averaging the predictions over persons:

1. Use the trained machine learning method to make a prediction $\hat{y}_{i}$ for each person $i$ for the position $a$ on predictor $x_{j}$, using the observed values of person $i$ for all other predictors.

$$
\operatorname{ICE}_{i j}(\mathbf{a})=\hat{f}^{M L}\left(x_{i 1}, \ldots, x_{i(j-1)}, \mathbf{a}, x_{i(j+1)}, \ldots, x_{i J}\right)
$$

2. -

3. To obtain $I C E_{i j}$ curves of predictor $x_{j}$, repeat this procedure for all other positions a and draw a curve for every person $i$.

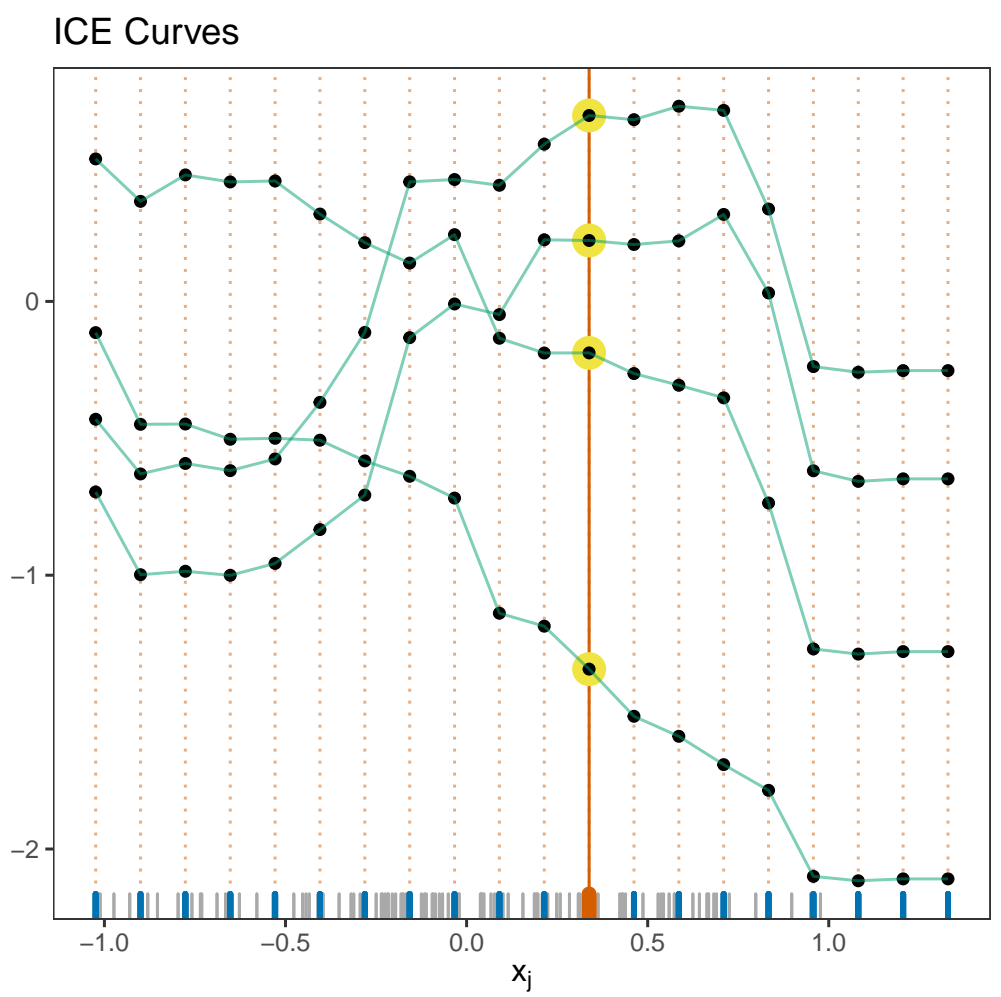




\section{B.3 Centered ICE}

In psychological research, centering very often means a shift on the x-axis, hence $x_{i j}-\bar{x}_{j}$. Here, centering takes place as a shift on the y-axis, so that all curves meet at a reference position $a^{\text {ref }}$. As a consequence, absolute height of the curves as well as the differences between the curves cannot be interpreted anymore. What remains for interpretation, are the slopes of each curve. To illustrate how centering in the direction of the y-axis affects intercepts and interindividual differences, we illustrate centered ICE curves for three different reference positions $a^{\text {ref }}$ : the minimum, the maximum, and the median of $x_{j}$.

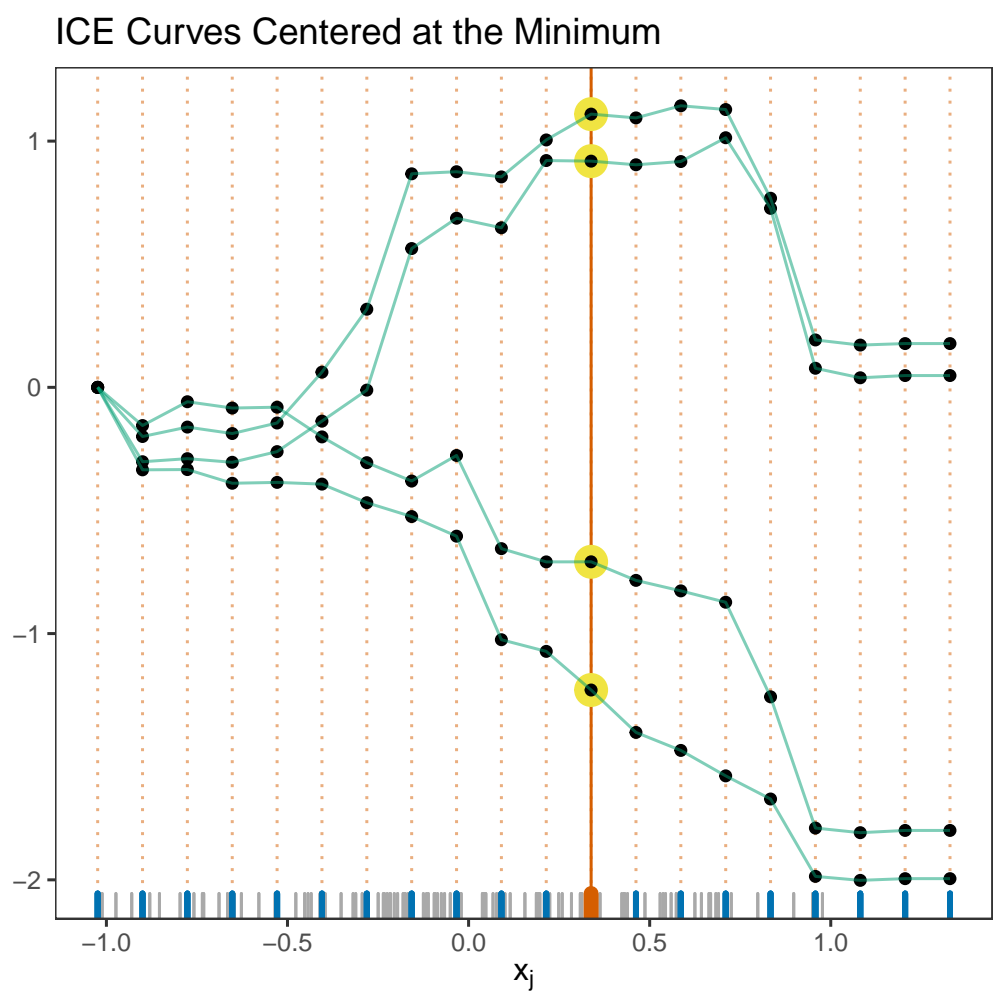




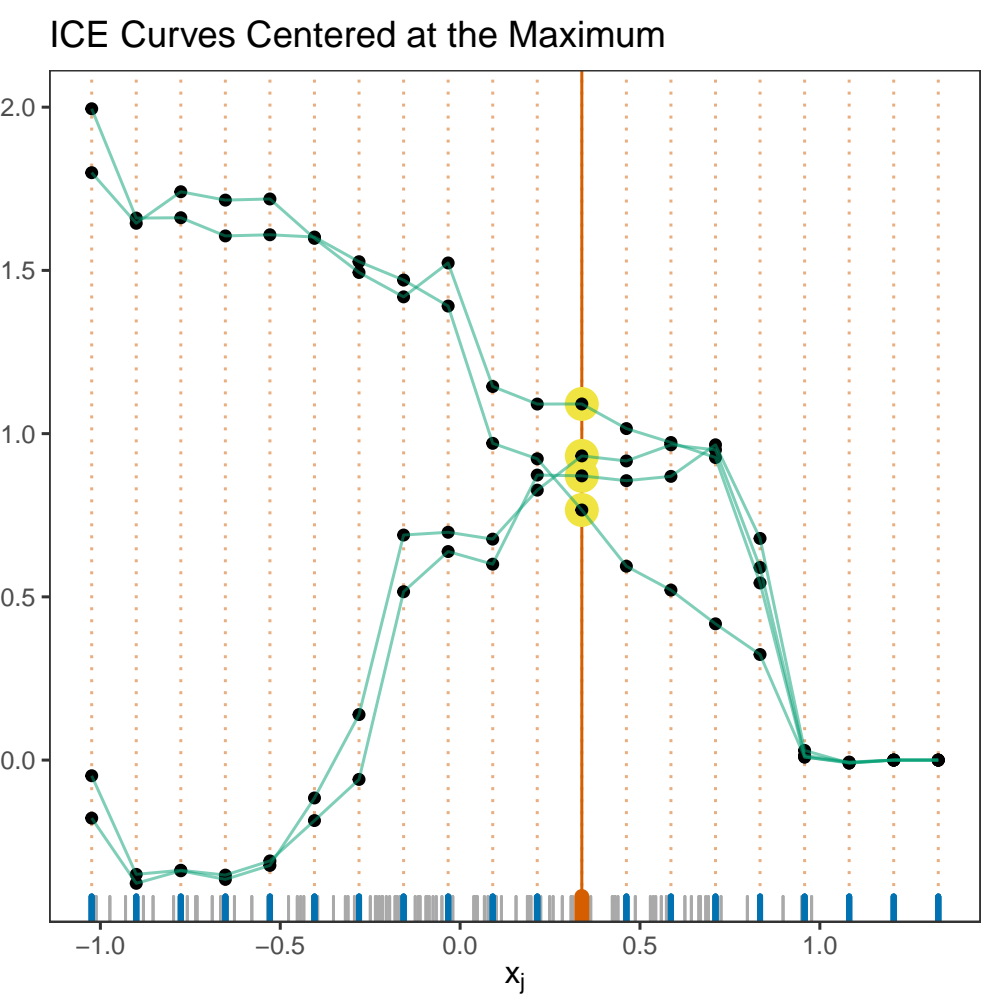

ICE Curves Centered at the Median

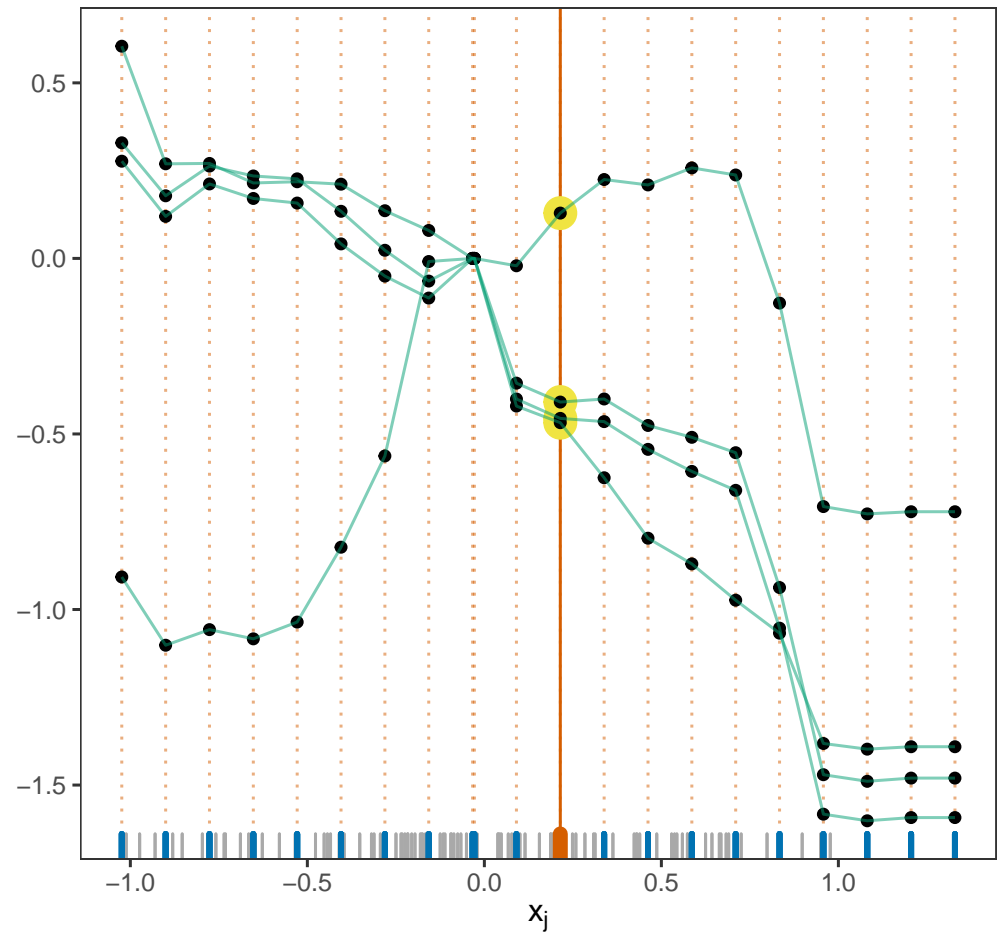




\section{B.4 Accumulated Local Effects}

1. The range of predictor $x_{j}$ is split in $K_{j}$ intervals $A_{j}^{(1)}, \ldots, A_{j}^{\left(K_{j}\right)}$ where each interval $k$ has an upper $\left(a_{j}^{(k)}\right)$ and a lower $\left(a_{j}^{(k-1)}\right)$ interval boundary, so that $A_{j}^{(k)}=\left[a_{j}^{(k-1)}, a_{j}^{(k)}\right]$

2. For $x_{i j}$ that are located within an interval $A_{j}^{(k)}\left(x_{i j} \in A_{j}^{(k)}\right)$, predictions at the lower interval boundary are subtracted from predictions at the upper interval boundary.

$$
\begin{aligned}
\Delta_{i}\left(A_{j}^{(k)}\right) & =\hat{f}^{M L}\left(x_{i 1}, \ldots, x_{i(j-1)}, a_{j}^{(k)}, x_{i(j+1)}, \ldots, x_{i J}\right) \\
& -\hat{f}^{M L}\left(x_{i 1}, \ldots, x_{i(j-1)}, a_{j}^{(k-1)}, x_{i(j+1)}, \ldots, x_{i J}\right)
\end{aligned}
$$

3. The prediction differences $\Delta_{i}\left(A_{j}^{(k)}\right)$ are averaged across all $n^{(k)}$ persons located in each interval.

$$
\bar{\Delta}\left(A_{j}^{(k)}\right)=\frac{1}{n^{(k)}} \sum_{x_{i j} \in A_{j}^{(k)}} \Delta_{i}\left(A_{j}^{(k)}\right)
$$

\section{Local Effect}

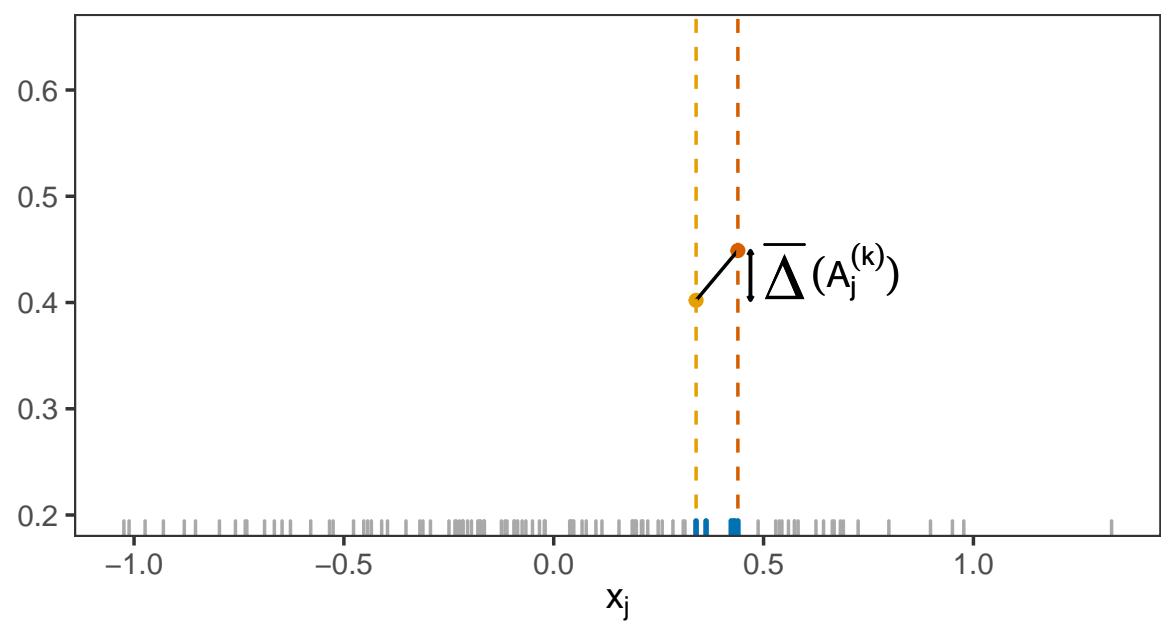

4. In order to describe the shape of the effect of $x_{j}$ on the outcome, the averaged prediction differences are accumulated up to the $k^{\text {th }}$ interval (see Apley \& Zhu, 2020).

$$
A L E^{\mathrm{unc}}\left(A_{j}^{(k)}\right)=\sum_{k^{*}=1}^{k} \bar{\Delta}\left(A_{j}^{\left(k^{*}\right)}\right)
$$

5. Finally, ALE effects are shifted vertically by subtracting a constant $C_{j}$ so that, averaged across persons, ALE effects are zero. Formally, the constant is a weighted average of each grid interval's ALE effect ${ }^{19}$ :

$$
A L E\left(A_{j}^{(k)}\right)=A L E^{\mathrm{unc}}\left(A_{j}^{(k)}\right)-C_{j}
$$

with

$$
C_{j}=\frac{1}{N} \sum_{k=1}^{K} n^{(k)} A L E^{\mathrm{unc}}\left(A_{j}^{(k)}\right)
$$

\footnotetext{
${ }^{19}$ As the intervals in this example are based on quantiles of $x_{j}$ so that an equal number of persons is in each interval, they are not equally wide (default in the $R$ package iml).
} 


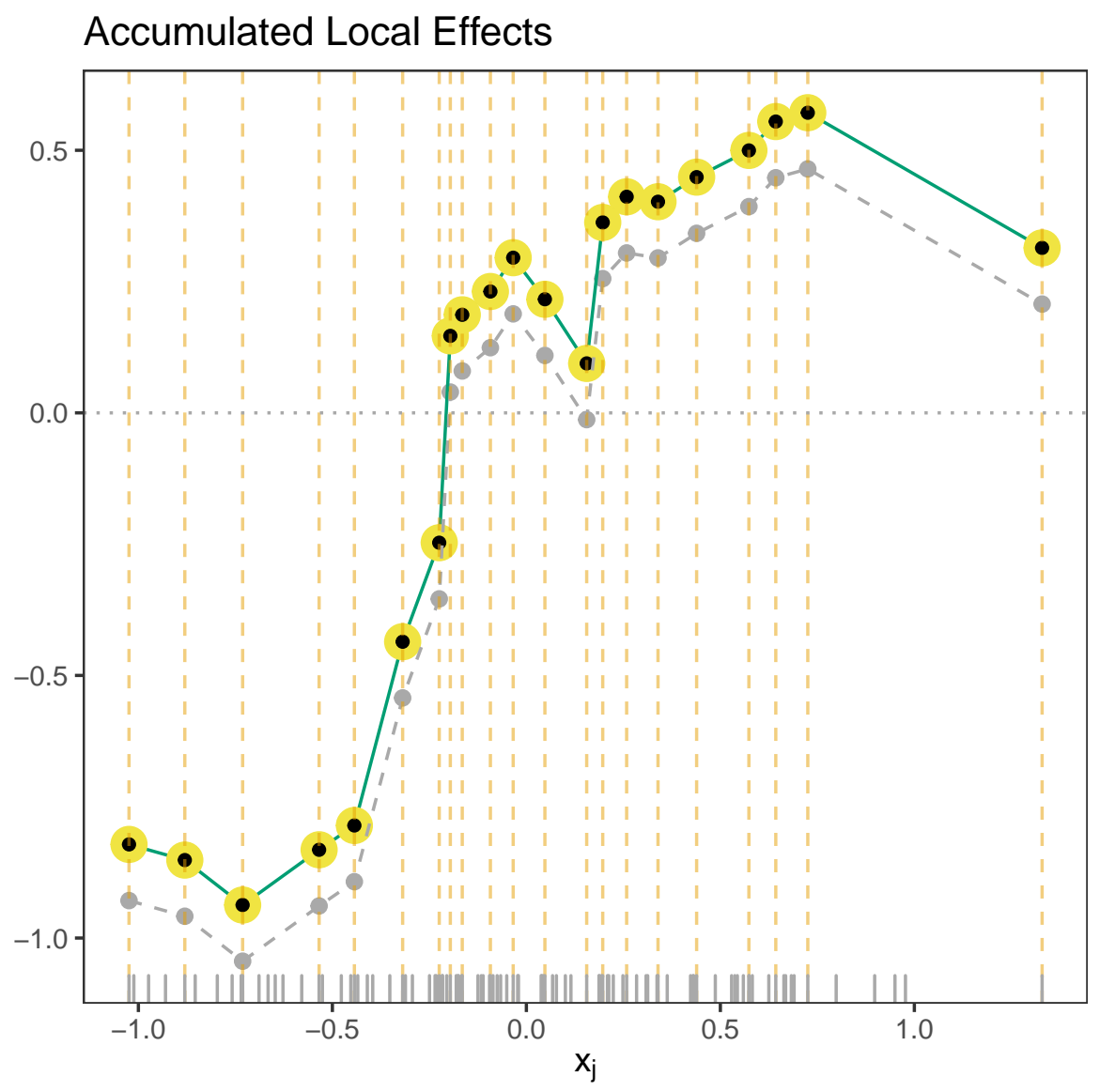




\section{Figures for Freed or Fixed Scales}

To illustrate the effect of fixing versus not fixing the scale limits of the interpretation technique visualizations, we show two exemplary alternatives for some visualizations in the main text here. For ALE curves, scales have been fixed in the main text (Figure 5), but in contrast ALE curves are shown here with free y-axis scales (Figure C.1). Optically, one may found the marginal effect of $x_{4}$ to be more pronounced in the version in the Appendix than in the version with fixed scales in the main text. Similarly, the colored and centered ICE furces have been fixed in the main text (Figure 14), but are shown here with free y-axis scales (Figure C.2).

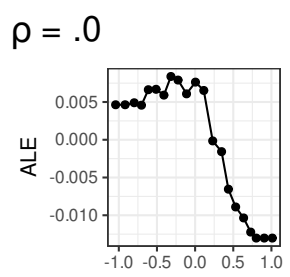

$\beta_{1}=0$

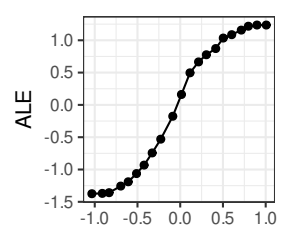

$\beta_{2}=2$

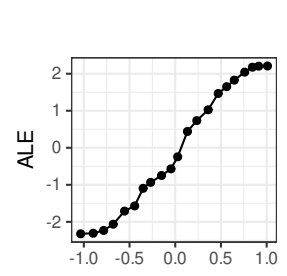

$\beta_{3}=3$

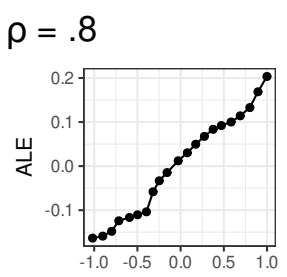

$\beta_{4}=0$

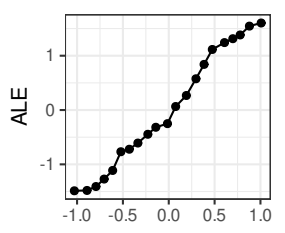

$\beta_{5}=2$

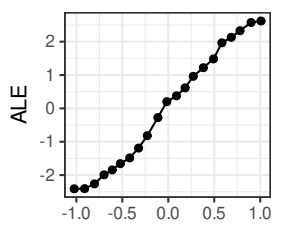

$\beta_{6}=3$

Figure C.1: ALE curves for data without correlations between $x_{1}, x_{2}$, and $x_{3}$, while $x_{4}, x_{5}$, and $x_{6}$ are block-correlated with $r=.8$. Data was generated under a linear regression model according to Equation 1 and fitted with a random forest. ALE plots with fixed scales are shown in Figure 5.

A
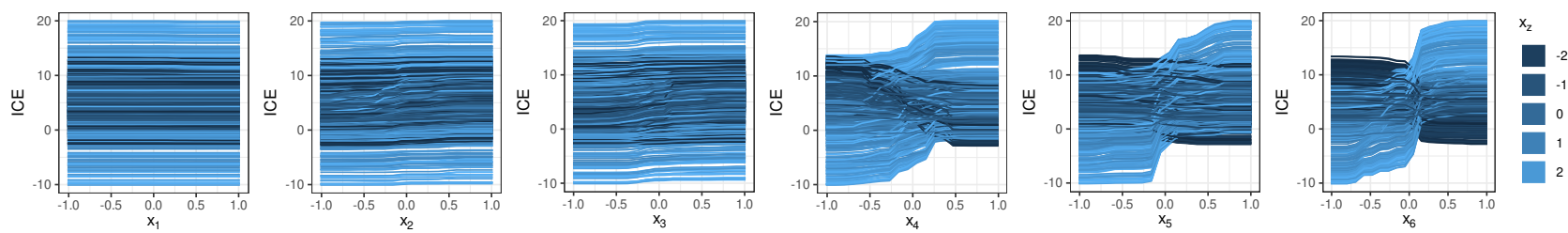

B
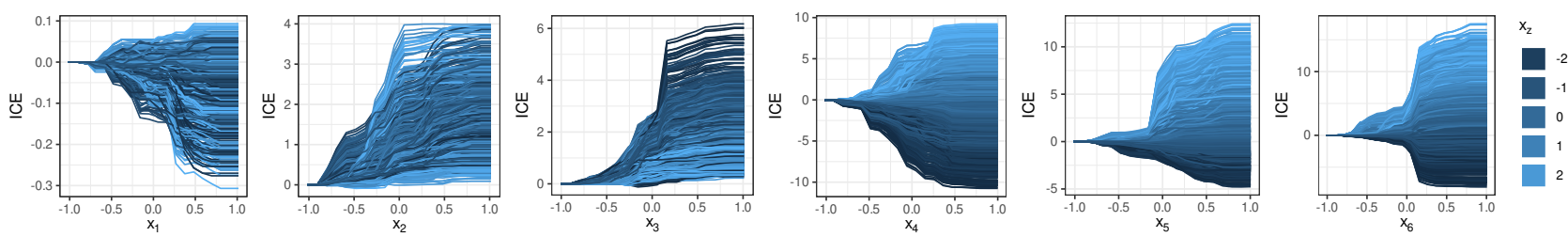

Figure C.2: ICE curves colored by $x_{z}$ (Panel A), colored and centered (Panel B) with data generated using the data generating process in Equation 2 for the random forest. Scales of all panels are free. ICE plots with fixed scales is shown in Figure 14. 


\section{Two-Dimensional PD Plots for a Decision Tree}
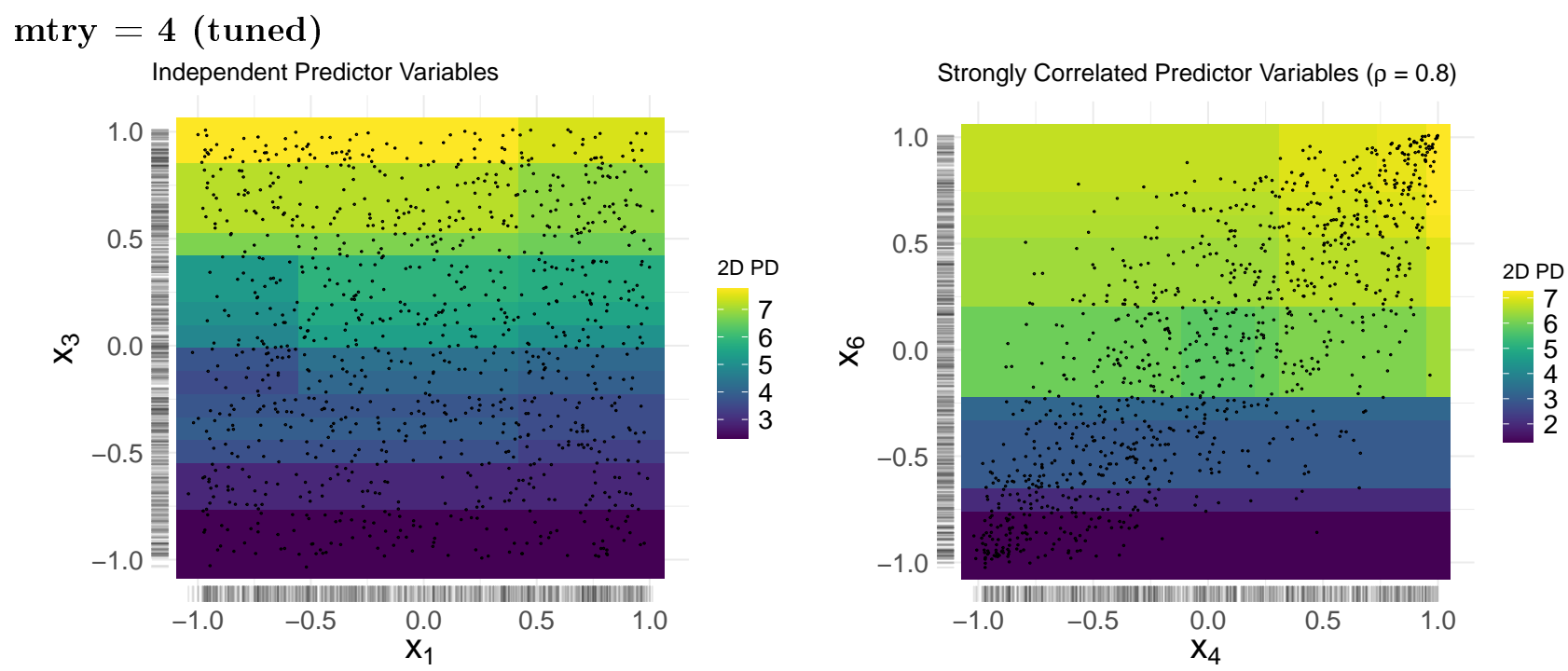

$$
\begin{aligned}
& \operatorname{mtry}=\mathbf{J} / \mathbf{3}=\mathbf{2} \\
& \text { Independent Predictor Variables }
\end{aligned}
$$

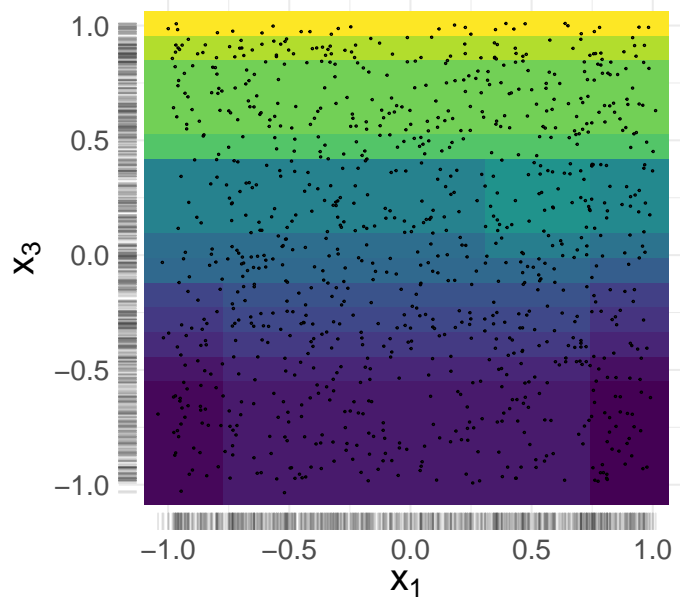

Strongly Correlated Predictor Variables $(\rho=0.8)$

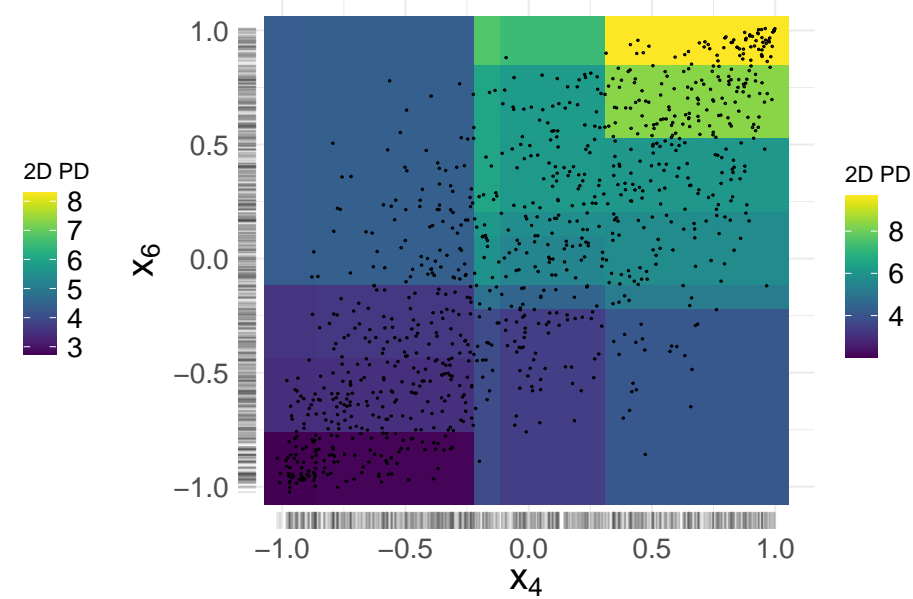

Figure D.1: Two-dimensional PD plots for a single decision tree from a random forest. Upper row: mtry $=4$ (value tuned in the pilot study and used in the main manuscript); lower row: $\mathrm{mtry}=2$ (typical default value: $\frac{J}{3}$ ). In contrast to the smooth prediction of the entire random forest in Figure 6 where the correlation did not lead to problems due to extrapolation, we get the impression that not only $x_{6}$, but also $x_{4}$ has an effect on the outcome when a single decision tree is used. 


\section{E Part II: Figures for the Neural Network}

\section{E.1 One- and Two-dimensional PD and ALE Plots}

(A) PD Plots

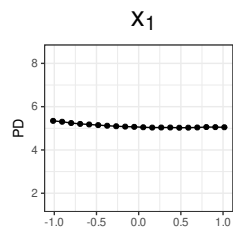

$\beta_{1}=0$

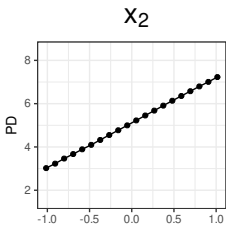

$\beta_{2}=2$

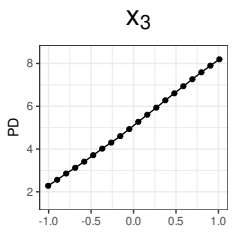

$\beta_{3}=3$

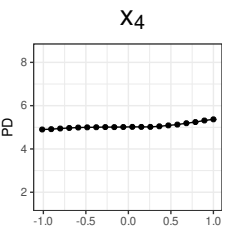

$\beta_{4}=0 ; \beta_{42}=3$

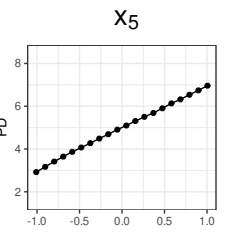

$\beta_{5}=2 ; \beta_{5 z}=3$

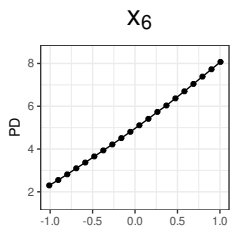

$\beta_{6}=3 ; \beta_{6 z}=3$

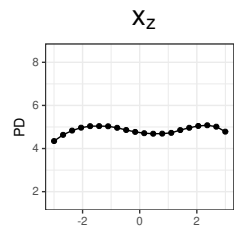

$\beta_{z}=0 ; \beta_{4 z}=\beta_{5 z}=\beta_{6 z}=3$

(B) ALE Plots

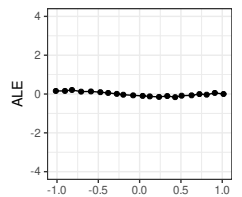

$\beta_{1}=0$

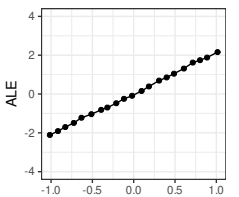

$\beta_{2}=2$

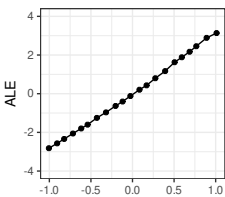

$\beta_{3}=3$

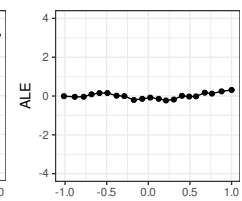

$\beta_{4}=0 ; \beta_{42}=3$

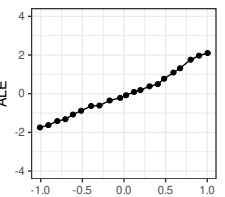

$\beta_{5}=2 ; \beta_{5 z}=3$

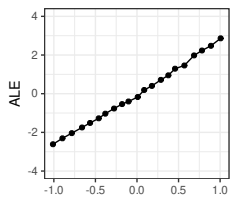

$\beta_{6}=3 ; \beta_{6 z}=3$

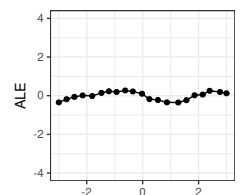

$\beta_{z}=0 ; \beta_{4 z}=\beta_{5 z}=\beta_{6 z}=3$

Figure E.1: PD (Panel A) and ALE curves (Panel B) with data generated using the data generating process in Equation 2 for the neural network. All visualizations in one row have fixed y-axis scales. Plots for the random forest are shown in Figure 9.

\section{(A) 2D PD Plots}
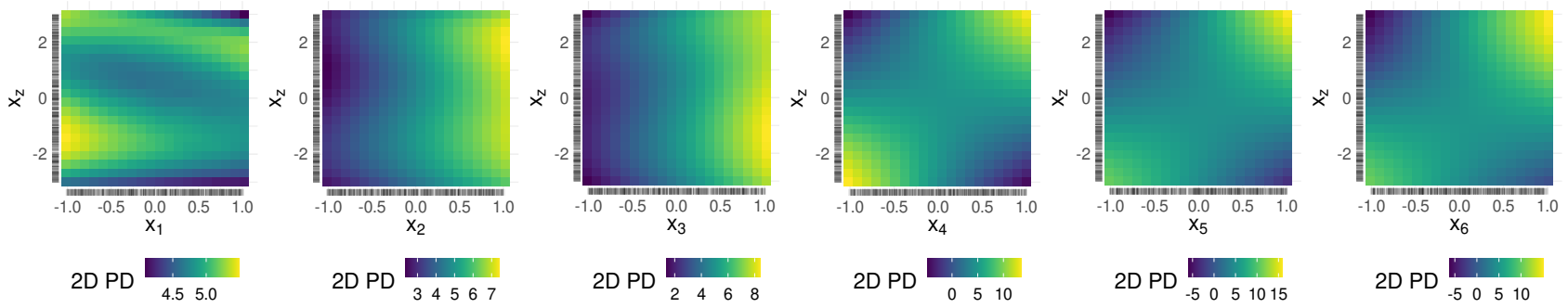

(B) 2D ALE Pplots

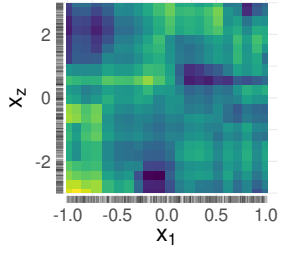

2D ALE $\begin{array}{lllllll}-2 & -1 & 0 & 1 & 2\end{array}$

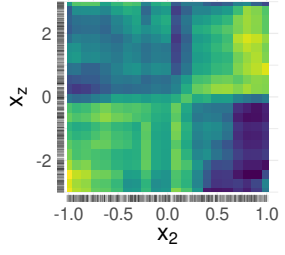

2D ALE $\begin{array}{rrrr}-1 & 0 & 1\end{array}$

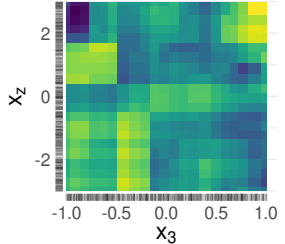

2D ALE $-2-1 \quad 0 \quad 12$

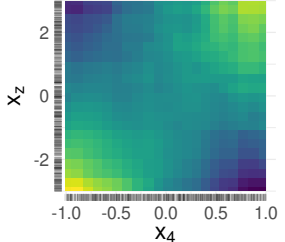

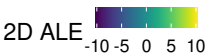

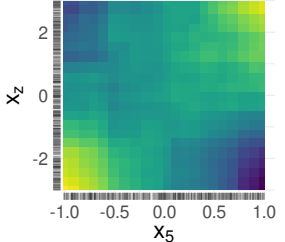

2D ALE $-10-5 \quad 0 \quad 5$

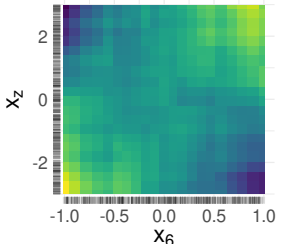

2D ALE $\begin{array}{llll}-6 & -3 & 0 & 3\end{array}$

Figure E.2: Panel A: Two-dimensional PD plots; Panel B: Two-dimensional ALE plots with data generated using the data generating process in Equation 2 for the neural network. ALE plots show interaction effects without main effects. Scale of the outcome is free between panels. Plots for the random forest are shown in Figure 11. 


\section{E.2 Colored and Centered ICE Plots}

\section{(A) Colored ICE Curves}
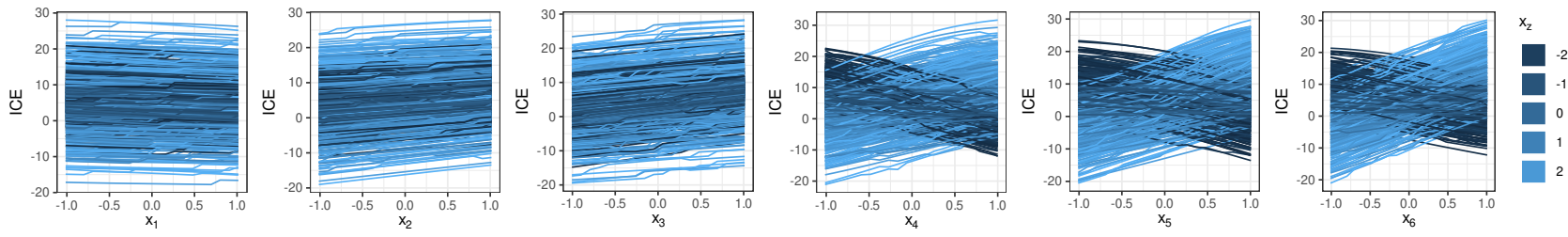

\section{(B) Centered ICE Curves}
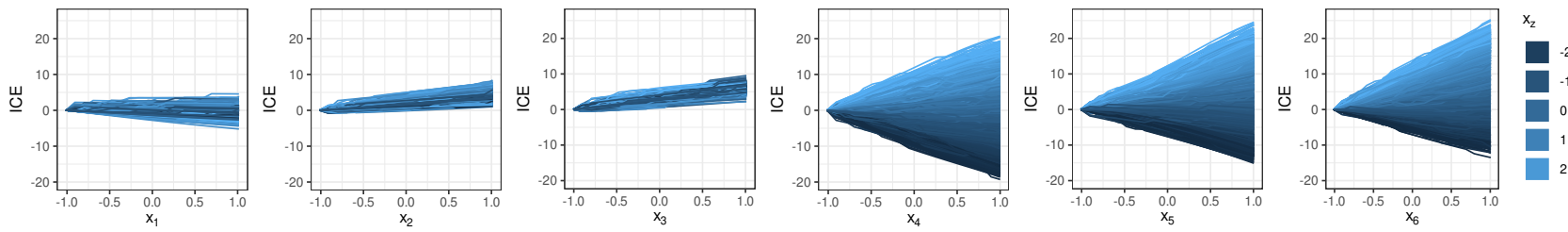

Figure E.3: ICE curves colored by $x_{z}$ (Panel A), colored and centered (Panel B) with data generated using the data generating process in Equation 2 for the neural network. Plots for the random forest are shown in Figure 14. 